\title{
Path Integral Representation For Schrödinger Operators with Bernstein Functions of the LAPLACIAN
}

\author{
Fumio Hiroshima \\ Faculty of Mathematics, Kyushu University \\ 6-10-1 Hakozaki, Fukuoka, 812-8581, Japan \\ hiroshima@math.kyushu-u.ac.jp \\ Takashi Ichinose \\ Department of Mathematics, Kanazawa University \\ Kanazawa, 920-11, Japan \\ ichinose@kenroku.kanazawa-u.ac.jp \\ József Lőrinczi \\ School of Mathematics, Loughborough University \\ Loughborough LE11 3TU, United Kingdom \\ J.Lorinczi@lboro.ac.uk
}

April 9, 2010

\begin{abstract}
Path integral representations for generalized Schrödinger operators obtained under a class of Bernstein functions of the Laplacian are established. The one-to-one correspondence of Bernstein functions with Lévy subordinators is used, thereby the role of Brownian motion entering the standard Feynman-Kac formula is taken here by subordinated Brownian motion. As specific examples, fractional and relativistic Schrödinger operators with magnetic field and spin are covered. Results on self-adjointness of these operators are obtained under conditions allowing for singular magnetic fields and singular external potentials as well as arbitrary integer and half-integer spin values. This approach also allows to propose a notion of generalized Kato class for which hypercontractivity of the associated generalized Schrödinger semigroup is shown. As a consequence, diamagnetic and energy comparison inequalities are also derived.
\end{abstract}




\section{Introduction}

\subsection{Context and motivation}

Feynman-Kac-type formulae prove to be a useful device in the analysis of spectral properties of a wide class of self-adjoint operators. Besides their prolific uses in the physics literature, functional integration poses remarkable new mathematical problems which can be addressed in terms of modern stochastic analysis.

The Feynman-Kac formula is a functional integral representation of the kernel of the semigroup generated by the Schrödinger operator

$$
H=\frac{1}{2} \mathrm{p}^{2}+V
$$

for which it was originally derived. Here $\mathrm{p}=-i \nabla$ is the momentum operator and $V$ is a potential. The Laplacian gives rise to an integral representation of the kernel of $e^{-t H}$ in terms of the Wiener measure, while $V$ introduces a density with respect to it. This implies that the ground state and various other properties of $H$ can be analyzed by running a Brownian motion under the potential $V$. Standard references on applications to the spectral analysis of Schrödinger operators include [Lie73, Lie80, Shi87, Sim82, with updated bibliography in Sim04]. We also refer to [DC00] for an approach with the Feynman-Kac formula. While functional integration can be extended to include several other operators also covering quantum field models (see [LHB09] and references therein), the analysis based on random processes having almost surely continuous paths remained a basic feature.

In the mathematical physics literature there appear to be relatively few systematic attempts in going beyond continuous paths to replace them with càdlàg paths (rightcontinuous with left limits), also allowing jump discontinuities. On the other hand, such more general Lévy processes than Brownian motion prove to be useful in describing important features such as spin in terms of path measures. Another source of problems leading to paths with jump discontinuities are models featuring fractional Laplacians.

The aim of the present paper is to construct path integral representations for generalized Schrödinger operators including both non-relativistic and relativistic Schrödinger operators with vector potentials and spin. We propose a thorough study of this problem, extending the methods developed in [HL08 to the case of Lévy processes with càdlàg paths.

By a generalized Schrödinger operator here we mean a Schrödinger operator in 
which the Laplacian is replaced by a suitable pseudo-differential operator. Namely, instead of the operator

$$
\frac{1}{2}(\sigma \cdot(\mathrm{p}-a))^{2}+V
$$

studied in [HL08, where $\sigma=\left(\sigma_{1}, \sigma_{2}, \sigma_{3}\right)$ are the Pauli matrices and $a$ is a vector potential, we consider a class of general self-adjoint operators of the form

$$
\Psi\left(\frac{1}{2}(\sigma \cdot(\mathrm{p}-a))^{2}\right)+V
$$

where $\Psi$ is a Bernstein function on the positive semi-axis (see below). In particular, this class includes not only relativistic Schrödinger operators

$$
\sqrt{(\sigma \cdot(\mathrm{p}-a))^{2}+m^{2}}-m+V
$$

but also more general fractional Schrödinger operators

$$
\left(\frac{1}{2}(\sigma \cdot(\mathrm{p}-a))^{2}\right)^{\alpha}+V
$$

with $\alpha \in(0,1)$. The vector potential plays the role of magnetic field in appropriate contexts, however, we will use this terminology for all cases we consider, even when they may have other interpretations.

The application of functional integral techniques to relativistic Schrödinger operators, without magnetic field or spin, has been earlier on addressed in [CMS90]. The process involved is closely related to $1 / 2$-stable processes, which can be understood in terms of a first hitting time process of Brownian motion. In the interesting papers ALS83, ARS91] a path integral for relativistic Schrödinger operators with vector potential and spin 1/2 is presented, however, in a non-rigorous language. A functional integral representation also has been established for the Schrödinger semigroup with vector potential in [ITa86], applied in [Ich87] and completed in [Ich94], where, however, the operator concerned was a pseudo-differential operator associated with the symbol of the classical relativistic Hamiltonian defined through Weyl quantization. It should be noted that the terms in (1.3)-(1.5) involving a vector potential cannot be defined as pseudodifferential operators associated with simple and plain symbols. A further step has been made by addressing various problems of potential theory and heat kernel estimates of more general $\alpha$-stable processes [BB99, BJ07, BKM06, CS97, Ryz02, KS06, GR07]; see also the influential work [Bak87] involving the Cauchy process. Such processes relate 
with fractional Schrödinger operators

$$
\left(\frac{1}{2} \mathrm{p}^{2}\right)^{\alpha}+V
$$

and are motivated by further models of physics, chemistry, biology and, more recently, financial mathematics BG90, BBACT02, EK95, MK04].

Fractional Schrödinger operators and stable processes provide just one special case of a sensible class of extensions. In the present paper we consider generalized Schrödinger operators obtained as Bernstein functions of the Laplacian to which we add an external potential $V$, and in various versions, a vector potential and a contribution from a spin operator. In a sense, this is the greatest desirable generality as Bernstein functions with vanishing right limits at the origin stand in a one-to-one correspondence with Lévy subordinators. Subordinators are random processes with jump discontinuities and can be uniquely described by specifying two parameters, the Lévy measure accounting for the jumps, and the drift function accounting for the continuous component of the paths. Given a Bernstein function $\Psi$ and a generalized Schrödinger operator $H^{\Psi}$ thereby obtained, the properties of the semigroup $e^{-t H^{\Psi}}$ can now be analyzed in terms of a subordinated Brownian motion $B_{T_{t}^{\Psi}}$. Here $T_{t}^{\Psi}$ is the Lévy subordinator uniquely associated with $\Psi$. Roughly speaking, $B_{T_{t}^{\Psi}}$ is a càdlàg process which samples Brownian paths at random times distributed by the law of $T_{t}^{\Psi}$.

\subsection{Main results}

Throughout this paper we will use the following conditions on the vector potential.

Assumption 1.1 The vector potential $a=\left(a_{1}, \ldots, a_{d}\right)$ is a vector-valued function whose components $a_{\mu}, \mu=1, \ldots, d$, are real-valued functions. Furthermore, we consider the following regularity conditions:

(A1) $a \in\left(L_{\text {loc }}^{2}\left(\mathbb{R}^{d}\right)\right)^{d}$.

(A2) $a \in\left(L_{\mathrm{loc}}^{2}\left(\mathbb{R}^{d}\right)\right)^{d}$ and $\nabla \cdot a \in L_{\mathrm{loc}}^{1}\left(\mathbb{R}^{d}\right)$.

(A3) $a \in\left(L_{\mathrm{loc}}^{4}\left(\mathbb{R}^{d}\right)\right)^{d}$ and $\nabla \cdot a \in L_{\mathrm{loc}}^{2}\left(\mathbb{R}^{d}\right)$.

(A4) $d=3, a \in\left(L_{\mathrm{loc}}^{4}\left(\mathbb{R}^{3}\right)\right)^{3}, \nabla \cdot a \in L_{\mathrm{loc}}^{2}\left(\mathbb{R}^{3}\right)$ and $\nabla \times a \in\left(L_{\mathrm{loc}}^{2}\left(\mathbb{R}^{3}\right)\right)^{3}$. 
Since we discuss several variants of Schrödinger operators, different by whether they do or do not include spin, it is appropriate to explain here the notation. We define the spinless operator through a quadratic form for $a$ satisfying (A1) and denote it by

$$
h=\frac{1}{2}(\mathrm{p}-a)^{2} \quad(\text { with no spin })
$$

A Schrödinger operator with spin $1 / 2$ also is defined through a quadratic form and will be denoted by

$$
h_{1 / 2}=\frac{1}{2}(\sigma \cdot(\mathrm{p}-a))^{2} \quad(\text { with spin }) .
$$

Using a suitable unitary map, we transform $h_{1 / 2}$ on the space $L^{2}\left(\mathbb{R}^{3} ; \mathbb{C}^{2}\right)=L^{2}\left(\mathbb{R}^{3}\right) \otimes \mathbb{C}^{2}$ to a self-adjoint operator $h_{\mathbb{Z}_{2}}$ on $L^{2}\left(\mathbb{R}^{3} \times \mathbb{Z}_{2}\right)$. Here $\mathbb{Z}_{2}=\{-1,1\}$ describes the state space of a two-valued spin variable. Furthermore, we generalize spin from $\mathbb{Z}_{2}$ to $\mathbb{Z}_{p}$ and denote a so obtained Schrödinger operator by

$$
h_{\mathbb{Z}_{p}} \quad \text { (with generalized spin) }
$$

acting on $L^{2}\left(\mathbb{R}^{d} \times \mathbb{Z}_{p}\right)$, for $d \geq 1$ and $p \geq 2$. The relativistic versions of (1.7) and (1.8) will be denoted by

$$
\begin{aligned}
& h^{\mathrm{rel}}=\sqrt{(\mathrm{p}-a)^{2}+m^{2}}-m, \quad m \geq 0 \\
& h_{1 / 2}^{\mathrm{rel}}=\sqrt{(\sigma \cdot(\mathrm{p}-a))^{2}+m^{2}}-m, \quad m \geq 0 .
\end{aligned}
$$

In this paper we will consider generalized versions of (1.10). Let $\Psi$ be a Bernstein function. Our main objects are

$$
\begin{aligned}
& \left.H^{\Psi}=\Psi(h)+V \quad \text { (with no spin }\right) \\
& \left.H_{\mathbb{Z}_{p}}^{\Psi}=\Psi\left(h_{\mathbb{Z}_{p}}\right)+V \quad \text { (with generalized spin }\right)
\end{aligned}
$$

In particular,

$$
\Psi(u)=\sqrt{2 u+m^{2}}-m
$$

corresponds to (1.10). Under Assumptions (A2) (resp. (A3)), we will show that $C_{0}^{\infty}\left(\mathbb{R}^{d}\right)$ is a form core (resp. operator core) of both $\Psi(h)$ and $\Psi\left(h_{\mathbb{Z}_{p}}\right)$. This is the content of Theorems 3.3 and 5.1 below.

The key results of this paper are the functional integral representations of $e^{-t H^{\Psi}}$ and $e^{-t H_{\mathbb{Z}_{p}}^{\Psi}}$ derived under Assumption (A2) for bounded potentials $V$. They are presented in Theorems 3.8 and 5.11, respectively. These are then further generalized to more 
singular potentials in Theorems 3.15 and 5.15. Recall that the standard FeynmanKac-Itô formula says that

$$
\left(f, e^{-t(h+V)} g\right)=\int_{\mathbb{R}^{d}} d x \mathbb{E}_{P}^{x}\left[\overline{f\left(B_{0}\right)} g\left(B_{t}\right) e^{-i \int_{0}^{t} a\left(B_{s}\right) \circ d B_{s}} e^{-\int_{0}^{t} V\left(B_{s}\right) d s}\right],
$$

with $d$-dimensional Brownian motion $\left(B_{t}\right)_{t \geq 0}$ on Wiener space $\left(\Omega_{P}, \mathscr{F}_{P}, P^{x}\right)$, where the stochastic integral in the exponent is Stratonovich integral. For $H^{\Psi}=\Psi(h)+V$ this formula modifies to (see Theorem 3.15 below)

$$
\left(f, e^{-t(\Psi(h)+V)} g\right)=\int_{\mathbb{R}^{d}} d x \mathbb{E}_{P \times \nu}^{x, 0}\left[\overline{f\left(B_{0}\right)} g\left(B_{T_{t}^{\Psi}}\right) e^{-i \int_{0}^{T_{t}^{\Psi}} a\left(B_{s}\right) \circ d B_{s}} e^{-\int_{0}^{t} V\left(B_{T_{s}^{\Psi}}\right) d s}\right]
$$

where $T_{t}^{\Psi}$ is the Lévy subordinator on a probability space $\left(\Omega_{\nu}, \mathscr{F}_{\nu}, \nu\right)$ associated with $\Psi$. In particular, it should be noted that the integrands change as

$$
\exp \left(-i \int_{0}^{t} a\left(B_{s}\right) \circ d B_{s}\right) \rightarrow \exp \left(-i \int_{0}^{T_{t}^{\Psi}} a\left(B_{s}\right) \circ d B_{s}\right)
$$

and

$$
\exp \left(-\int_{0}^{t} V\left(B_{s}\right) d s\right) \rightarrow \exp \left(-\int_{0}^{t} V\left(B_{T_{s}^{\Psi}}\right) d s\right) .
$$

A similar situation occurs in the case including a generalized spin, see Theorem 5.15 below. By means of these formulae we are able to extend the definition of generalized Schrödinger operators $H^{\Psi}$ and $H_{\mathbb{Z}_{p}}^{\Psi}$ to the case of external potentials having singularities.

Having the functional integral representations at hand allows us to construct a strongly continuous symmetric Feynman-Kac semigroup for a large class of potentials $V$ which we call $\Psi$-Kato class. This will be dealt with in Theorem 4.11. The generator of this semigroup can be identified as a self-adjoint operator, which we denote by

$$
K^{\Psi} \quad \text { (with } \Psi \text {-Kato class potential). }
$$

This offers then a notion of generalized Schrödinger operator with vector potential for $\Psi$-Kato potentials. As a further result, we show hypercontractivity of the semigroup $e^{-t K^{\Psi}}$ in Theorem 4.13.

As corollaries of Theorems 3.8 and 5.11, by choosing $\Psi(u)=\sqrt{2 u+m^{2}}-m$ mentioned above we obtain the functional integral representations of the relativistic Schrödinger operators

$$
\begin{array}{ll}
h^{\mathrm{rel}}+V=\sqrt{(\mathrm{p}-a)^{2}+m^{2}}-m+V & \text { (with no spin), } \\
h_{1 / 2}^{\mathrm{rel}}+V=\sqrt{(\sigma \cdot(\mathrm{p}-a))^{2}+m^{2}}-m+V & (\text { with spin } 1 / 2)
\end{array}
$$


in Theorems 6.1 and 6.4, respectively, and derive energy comparison inequalities. Our results improve and generalize those of [BHL00, CMS90, ITa86, ALS83, ARS91, Sim82, GV81. Further applications to relativistic quantum field theory are discussed in [Hir09, HS09, Lor09a, Lor09b.

The paper is organized as follows. In Section 2 we discuss the details of the relationship between Bernstein functions $\Psi$ and Lévy subordinators $\left(T_{t}^{\Psi}\right)_{t \geq 0}$. In Section 3 we consider the spinless case. We establish the functional integral representation for their semigroup and obtain diamagnetic inequalities. Furthermore, we show essential self-adjointness of $\Psi(h)$ on $C_{0}^{\infty}\left(\mathbb{R}^{d}\right)$. In Section 4 , we define the space of $\Psi$-Kato class potentials and discuss their relationship with the Lévy measure of the associated subordinators. Also, we prove hypercontractivity of the generalized Schrödinger semigroups obtained for this class. In Section 5 we consider generalized Schrödinger operators with spin. We extend \pm 1 spins to spins of $p$ possible orientations by describing them in terms of the cyclic group of the $p$ th roots of unity. This gives rise to a random process driven by a weighted sum of $p$ independent Poisson variables of intensity 1 . As a corollary, we derive diamagnetic inequalities. Finally, in Section 6 we give a functional integral representation of the relativistic Schrödinger operator with and without spin as a special case.

\section{Bernstein functions and Lévy subordinators}

We start by considering some basic facts on Bernstein functions and their connection with subordinators. For standard definitions and results on Bernstein functions we refer to [Boc55, BF73], for Lévy processes to [Sat99], to [Ber99] for a detailed study on Lévy subordinators, and to [Huf69, SV09] for details on subordinated Brownian motion.

Bernstein functions appear in the analysis of convolution semigroups, in particular they are a key concept in Bochner's theory of subordination.

Definition 2.1 (Bernstein function) Let

$$
\mathscr{B}=\left\{f \in C^{\infty}((0, \infty)) \mid f(x) \geq 0 \text { and }(-1)^{n}\left(\frac{d^{n} f}{d x^{n}}\right)(x) \leq 0 \text { for all } n=1,2, \ldots,\right\} \text {. }
$$

An element of $\mathscr{B}$ is called a Bernstein function. We also define the subclass

$$
\mathscr{B}_{0}=\left\{f \in \mathscr{B} \mid \lim _{u \rightarrow 0+} f(u)=0\right\} .
$$


Bernstein functions are positive, increasing and concave. $\mathscr{B}$ is a convex cone containing the nonnegative constants. Examples of functions in $\mathscr{B}_{0}$ include $\Psi(u)=c u^{\alpha}$, $c \geq 0,0<\alpha \leq 1$, and $\Psi(u)=1-e^{-a u}, a \geq 0$.

A real-valued function $f$ on $(0, \infty)$ is a Bernstein function if and only if $g_{t}:=e^{-t f}$ is a completely monotone function for all $t>0$, i.e., exactly when $(-1)^{n} \frac{d^{n} g_{t}}{d x^{n}} \geq 0$, for all integers $n \geq 0$. On the other hand, a result by Bernstein says that a function is completely monotone if and only if it is the Laplace transform of a positive measure, which for each such function is unique. This leads to the following integral representation of Bernstein functions.

Definition 2.2 (Class $\mathscr{L}$ ) Let $\mathscr{L}$ be the set of Borel measures $\lambda$ on $\mathbb{R} \backslash\{0\}$ such that

(1) $\lambda((-\infty, 0))=0$;

(2) $\int_{\mathbb{R} \backslash\{0\}}(y \wedge 1) \lambda(d y)<\infty$.

Note that each $\lambda \in \mathscr{L}$ satisfies that $\int_{\mathbb{R} \backslash\{0\}}\left(y^{2} \wedge 1\right) \lambda(d y)<\infty$ so that $\lambda$ is a Lévy measure.

Denote $\mathbb{R}_{+}=[0, \infty)$. We give the integral representation of Bernstein functions with vanishing right limits at the origin.

Proposition 2.3 For every Bernstein function $\Psi \in \mathscr{B}_{0}$ there exists $(b, \lambda) \in \mathbb{R}_{+} \times \mathscr{L}$ such that

$$
\Psi(u)=b u+\int_{0}^{\infty}\left(1-e^{-u y}\right) \lambda(d y) .
$$

Conversely, the right hand side of (2.1) is in $\mathscr{B}_{0}$ for each pair $(b, \lambda) \in \mathbb{R}_{+} \times \mathscr{L}$.

For a given $\Psi \in \mathscr{B}_{0}$, the constant $b$ is uniquely determined by $b=\lim _{u \rightarrow \infty} \Psi(u) / u$. Moreover, since $\frac{d \Psi}{d u}=b+\int_{0}^{\infty} y e^{-y u} \lambda(d y)$ and $\frac{d \Psi}{d u}$ is a completely monotone function, the measure $\lambda$ is also uniquely determined; for details, see [BF73, Theorem 9.8]. Thus the map $\mathscr{B}_{0} \rightarrow \mathbb{R}_{+} \times \mathscr{L}, \Psi \mapsto(b, \lambda)$ is a one-to-one correspondence.

Next we consider a probability space $\left(\Omega_{\nu}, \mathscr{F}_{\nu}, \nu\right)$ given and the following special class of Lévy processes.

Definition 2.4 (Lévy subordinator) A random process $\left(T_{t}\right)_{t \geq 0}$ on $\left(\Omega_{\nu}, \mathscr{F}_{\nu}, \nu\right)$ is called a (Lévy) subordinator whenever 
(1) $\left(T_{t}\right)_{t \geq 0}$ is a Lévy process starting at 0 , i.e., $\nu\left(T_{0}=0\right)=1$;

(2) $T_{t}$ is almost surely non-decreasing in $t$.

Subordinators have thus independent and stationary increments, almost surely no negative jumps, and are of bounded variation. These properties also imply that they are Markov processes.

Let $\mathscr{S}$ denote the set of subordinators on $\left(\Omega_{\nu}, \mathscr{F}_{\nu}, \nu\right)$. In what follows we denote expectation by $\mathbb{E}_{m}^{x}[\cdots]=\int \cdots d m^{x}$ with respect to the path measure $m^{x}$ of a process starting at $x$.

Proposition 2.5 Let $\Psi \in \mathscr{B}_{0}$ or, equivalently, a pair $(b, \lambda) \in \mathbb{R}_{+} \times \mathscr{L}$ be given. Then there exists a unique $\left(T_{t}\right)_{t \geq 0} \in \mathscr{S}$ such that

$$
\mathbb{E}_{\nu}^{0}\left[e^{-u T_{t}}\right]=e^{-t \Psi(u)}
$$

Conversely, let $\left(T_{t}\right)_{t \geq 0} \in \mathscr{S}$. Then there exists $\Psi \in \mathscr{B}_{0}$, i.e., a pair $(b, \lambda) \in \mathbb{R}_{+} \times \mathscr{L}$ such that (2.2) is satisfied.

In particular, (2.1) coincides with the Lévy-Khintchine formula for Laplace exponents of subordinators.

By the above there is a one-to-one correspondence between $\mathscr{B}_{0}$ and $\mathscr{S}$, or equivalently, between $\mathscr{B}_{0}$ and $\mathbb{R}_{+} \times \mathscr{L}$. For clarity, we will use the notation $T_{t}^{\Psi}$ for the Lévy subordinator associated with $\Psi \in \mathscr{B}_{0}$.

Example 2.6 (Stable processes) Let $b=0,0<\alpha<1$ and $\lambda \in \mathscr{L}$ be defined by

$$
\lambda(d y)=\frac{\alpha}{\Gamma(1-\alpha)} \frac{1_{(0, \infty)}(y)}{y^{1+\alpha}} d y
$$

where $\Gamma$ denotes the Gamma function. Then $\Psi(u)=u^{\alpha} \in \mathscr{B}_{0}$ and the corresponding subordinator $T_{t}^{\Psi}$ is given by

$$
\mathbb{E}_{\nu}^{0}\left[e^{-u T_{t}^{\Psi}}\right]=e^{-t u^{\alpha}}
$$

Example 2.7 (First hitting time) Since $\Psi(u)=\sqrt{2 u+m^{2}}-m \in \mathscr{B}_{0}$ for $m \geq 0$, there exists $T_{t}^{\Psi} \in \mathscr{S}$ such that

$$
\mathbb{E}_{\nu}^{0}\left[e^{-u T_{t}^{\Psi}}\right]=\exp \left(-t\left(\sqrt{2 u+m^{2}}-m\right)\right) .
$$


This case is thus related to the one-dimensional $1 / 2$-stable process and it is known that the corresponding subordinator $T_{t}^{\Psi}$ can be represented as the first hitting time process

$$
T_{t}^{\Psi}=\inf \left\{s>0 \mid B_{s}+m s=t\right\}
$$

for one-dimensional Brownian motion $\left(B_{t}\right)_{t \geq 0}$. In this case, moreover, the distribution also is known exactly to be

$$
\rho(r, t)=\frac{t}{\sqrt{2 \pi r^{3}}} e^{m t} \exp \left(-\frac{1}{2}\left(\frac{t^{2}}{r^{2}}+m^{2} r\right)\right) .
$$

Example 2.8 (Hyperbolic Lévy motion) A specific case studied in mathematical finance EK95] is

$$
\Psi(u)=-\log \left(\frac{a K_{1}\left(\sqrt{a^{2}+b^{2} u^{2}}\right)}{K_{1}(a) \sqrt{a^{2}+b^{2} u^{2}}}\right), \quad a, b>0,
$$

where $K_{1}$ is the modified Bessel function of the third kind with index 1 . This is a purely discontinuous process with Lévy measure

$$
\lambda_{a, b}(d y)=\left(\frac{1}{\pi^{2} y} \int_{0}^{\infty} \frac{e^{-y \sqrt{2 x+(a / b)^{2}}}}{J_{1}^{2}(b \sqrt{2 x})+Y_{1}^{2}(b \sqrt{2 x})} \frac{d x}{x}+\frac{e^{-y}}{y}\right) 1_{(0, \infty)}(y) d y,
$$

where $J_{1}$ and $Y_{1}$ are the Bessel functions of the first and second kind, with index 1 .

\section{Spinless case}

\subsection{Generalized Schrödinger operators with no spin}

Now we define the class of generalized Schrödinger operators on $L^{2}\left(\mathbb{R}^{d}\right)$, which we consider in this paper. In order to cover interactions with a magnetic field we add a vector potential to the momentum operator. Let $\partial_{x_{\mu}}: \mathscr{D}^{\prime}\left(\mathbb{R}^{d}\right) \rightarrow \mathscr{D}^{\prime}\left(\mathbb{R}^{d}\right), \mu=1, \ldots, d$, denote the $\mu$ th derivative on the Schwartz distribution space $\mathscr{D}^{\prime}\left(\mathbb{R}^{d}\right)$. With the notation $\mathrm{p}=-i \nabla$ and $\nabla=\left(\partial_{x_{1}}, \ldots, \partial_{x_{d}}\right)$, the Schrödinger operator with vector potential $a$ is formally given by $\frac{1}{2}(\mathrm{p}-a)^{2}$. We will define it as a self-adjoint operator rigorously through a quadratic form.

Let $\mathrm{D}_{\mu}=\mathrm{p}_{\mu}-a_{\mu}, \mu=1, \ldots, d$. Define the quadratic form

$$
q(f, g)=\sum_{\mu=1}^{d}\left(\mathrm{D}_{\mu} f, \mathrm{D}_{\mu} g\right)
$$


with domain

$$
Q(q)=\left\{f \in L^{2}\left(\mathbb{R}^{d}\right) \mid \mathrm{D}_{\mu} f \in L^{2}\left(\mathbb{R}^{d}\right), \mu=1, \ldots, d\right\} .
$$

It can be seen that $Q(q)$ is complete with respect to the norm $\|f\|_{q}=\sqrt{q(f, f)+\|f\|^{2}}$ under Assumption (A1). Thus $q$ is a non-negative closed form and there exists a unique self-adjoint operator $h$ satisfying

$$
(h f, g)=q(f, g), \quad f \in D(h), \quad g \in Q(q),
$$

with domain

$$
D(h)=\left\{f \in Q(q) \mid q(f, \cdot) \in L^{2}\left(\mathbb{R}^{d}\right)^{\prime}\right\} .
$$

The self-adjoint operator $h$ is our main object in this section. We summarize some facts about the form core and operator core of $h$ [Sim79, LS81].

Proposition 3.1 (1) Let Assumption (A1) hold. Then $C_{0}^{\infty}\left(\mathbb{R}^{d}\right)$ is a form core of $h$. (2) Let Assumption (A3) hold. Then $C_{0}^{\infty}\left(\mathbb{R}^{d}\right)$ is an operator core for $h$.

Note that in case (2) of Proposition 3.1.

$$
h f=\frac{1}{2} \mathrm{p}^{2} f-a \cdot \mathrm{p} f+\left(-\frac{1}{2} a \cdot a-(\mathrm{p} \cdot a)\right) f .
$$

Definition 3.2 (Generalized Schrödinger operator with vector potential and bounded $V$ ) Let $\Psi \in \mathscr{B}_{0}$ and take Assumption (A1). Whenever $V$ is bounded we call

$$
H^{\Psi}=\Psi(h)+V
$$

generalized Schrödinger operator with vector potential a.

Note that $\Psi \geq 0$ and $\Psi(h)$ is defined through the spectral projection of the self-adjoint operator $h$. Furthermore, $H^{\Psi}$ is self-adjoint on the domain $D(\Psi(h))$ as $V$ is bounded.

\subsection{Essential self-adjointness}

Theorem 3.3 Take $\Psi \in \mathscr{B}_{0}$.

(1) Let Assumption (A3) hold. Then $C_{0}^{\infty}\left(\mathbb{R}^{d}\right)$ is an operator core of $\Psi(h)$.

(2) Let Assumption (A1) hold. Then $C_{0}^{\infty}\left(\mathbb{R}^{d}\right)$ is a form core of $\Psi(h)$. 
Proof. (1) Recall the representation (2.1). Since we have $\int_{0}^{1} y \lambda(d y)<\infty$ and $\int_{1}^{\infty} \lambda(d y)<\infty$ by Definition 2.2, there exist non-negative constants $c_{1}$ and $c_{2}$ such that $\Psi(u) \leq c_{1} u+c_{2}$ for all $u \geq 0$. This gives the bound

$$
\|\Psi(h) f\| \leq c_{1}\|h f\|+c_{2}\|f\|
$$

for all $f \in D(h)$. Hence $C_{0}^{\infty}\left(\mathbb{R}^{d}\right)$ is contained in $D(\Psi(h))$. Since $\Psi(h)$ is a non-negative self-adjoint operator, $\Psi(h)+1$ has a bounded inverse, and we use that $C_{0}^{\infty}\left(\mathbb{R}^{d}\right)$ is a core of $\Psi(h)$ if and only if $\Psi(h) C_{0}^{\infty}\left(\mathbb{R}^{d}\right)$ is dense in $L^{2}\left(\mathbb{R}^{d}\right)$. Let $g \in L^{2}\left(\mathbb{R}^{d}\right)$ and suppose that $(g,(\Psi(h)+1) f)=0$, for all $f \in C_{0}^{\infty}\left(\mathbb{R}^{d}\right)$. Then $C_{0}^{\infty}\left(\mathbb{R}^{d}\right) \ni f \mapsto(g, \Psi(h) f)=-(g, f)$ defines a continuous functional which can be extended to $L^{2}\left(\mathbb{R}^{d}\right)$. Thus $g \in D(\Psi(h))$ and $0=((\Psi(h)+1) g, f)$. Since $C_{0}^{\infty}\left(\mathbb{R}^{d}\right)$ is dense, we have $(\Psi(h)+1) g=0$, and hence $g=0$ since $\Psi(h)+1$ is one-to-one, proving the assertion.

(2) Note that $\left\|\Psi(h)^{1 / 2} f\right\|^{2} \leq c_{1}\left\|h^{1 / 2} f\right\|^{2}+c_{2}\|f\|^{2}$ for $f \in Q(h)=D\left(h^{1 / 2}\right)$, and $C_{0}^{\infty}\left(\mathbb{R}^{d}\right)$ is contained in $Q(\Psi(h))=D\left(\Psi(h)^{1 / 2}\right)$. Since $\Psi(h)^{1 / 2}+1$ has also bounded inverse, it is seen by the same argument as above that $C_{0}^{\infty}\left(\mathbb{R}^{d}\right)$ is a core of $\Psi(h)^{1 / 2}$ or a form core of $\Psi(h)$.

qed

\subsection{Singular magnetic fields}

Before constructing a functional integral representation of $e^{-t h}$, we extend stochastic integration to a class including $L_{\text {loc }}^{2}\left(\mathbb{R}^{d}\right)$ functions since the vector potentials we consider may be more singular.

Let $\left(B_{t}\right)_{t \geq 0}$ denote $d$-dimensional Brownian motion starting at $x \in \mathbb{R}^{d}$ on standard Wiener space $\left(\Omega_{P}, \mathscr{F}_{P}, P^{x}\right)$. Let $f$ be a $\mathbb{C}^{d}$-valued Borel measurable function on $\mathbb{R}^{d}$ such that

$$
\mathbb{E}_{P}^{x}\left[\int_{0}^{t}\left|f\left(B_{s}\right)\right|^{2} d s\right]<\infty .
$$

Then the stochastic integral $\int_{0}^{t} f\left(B_{s}\right) \cdot d B_{s}$ is defined as a martingale and the Itô isometry

$$
\mathbb{E}_{P}^{x}\left[\left.|| \int_{0}^{t} f\left(B_{s}\right) \cdot d B_{s}\right|^{2}\right]=\mathbb{E}_{P}^{x}\left[\int_{0}^{t}\left|f\left(B_{s}\right)\right|^{2} d s\right]
$$

holds. However, vector potentials a under (A.1) of Assumption 1.1 do not necessarily satisfy (3.7). As we show next, a stochastic integral can indeed be defined for a wider class of functions than (3.7), and then $\int_{0}^{t} f\left(B_{s}\right) \cdot d B_{s}$ will be defined as a local martingale 
instead of a martingale. This extension will allow us to derive a functional integral representation of $e^{-t h}$ with $a \in\left(L_{\text {loc }}^{2}\left(\mathbb{R}^{d}\right)\right)^{d}$.

Consider the following class of vector valued functions on $\mathbb{R}^{d}$.

Definition 3.4 We say that $f=\left(f_{1}, \ldots, f_{d}\right) \in \mathscr{E}_{\text {loc }}$ if and only if for all $t \geq 0$

$$
P^{x}\left(\int_{0}^{t}\left|f\left(B_{s}\right)\right|^{2} d s<\infty\right)=1 .
$$

Let $R_{n}(\omega)=n \wedge \inf \left\{t \geq\left. 0\left|\int_{0}^{t}\right| f\left(B_{s}(\omega)\right)\right|^{2} d s \geq n\right\}$ be a sequence of stopping times with respect to the natural filtration $\mathscr{F}_{t}^{P}=\sigma\left(B_{s}, 0 \leq s \leq t\right)$. Define

$$
f_{n}(s, \omega)=f\left(B_{s}(\omega)\right) 1_{\left\{R_{n}(\omega)>s\right\}} .
$$

Each of these functions satisfies $\int_{0}^{\infty}\left|f_{n}(s, \omega)\right|^{2} d s=\int_{0}^{R_{n}}\left|f_{n}(s, \omega)\right|^{2} d s \leq n$. In particular, we have $\mathbb{E}_{P}^{x}\left[\int_{0}^{t}\left|f_{n}\right|^{2} d s\right]<\infty$ and thus $\int_{0}^{t} f_{n} \cdot d B_{s}$ is well defined. Moreover, it can be seen that

$$
\int_{0}^{t \wedge R_{m}} f_{n}(s, \omega) \cdot d B_{s}=\int_{0}^{t} f_{m}(s, \omega) \cdot d B_{s}
$$

for $m<n$.

Definition 3.5 For $f \in \mathscr{E}_{\text {loc }}$ we define the integral

$$
\int_{0}^{t} f\left(B_{s}\right) \cdot d B_{s}:=\int_{0}^{t} f_{n}(s, \omega) \cdot d B_{s}, \quad 0 \leq t \leq R_{n} .
$$

This definition is consistent with (3.10).

Lemma 3.6 $\mathscr{E}_{\mathrm{loc}}$ has properties below:

(1) Let $f \in \mathscr{E}_{\text {loc }}$. Suppose that a sequence of step functions $f_{n}, n=1,2, \ldots$, satisfies $\int_{0}^{t}\left|f_{n}\left(B_{s}\right)-f\left(B_{s}\right)\right|^{2} d s \rightarrow 0$ in probability as $n \rightarrow \infty$. Then

$$
\lim _{n \rightarrow \infty} \int_{0}^{t} f_{n}\left(B_{s}\right) \cdot d B_{s}=\int_{0}^{t} f\left(B_{s}\right) \cdot d B_{s} \quad \text { in probability. }
$$

(2) $\left(L_{\mathrm{loc}}^{2}\left(\mathbb{R}^{d}\right)\right)^{d} \subset \mathscr{E}_{\mathrm{loc}}$.

(3) Let $a \in\left(L_{\text {loc }}^{2}\left(\mathbb{R}^{d}\right)\right)^{d}$ and $\nabla \cdot a \in L_{\text {loc }}^{1}\left(\mathbb{R}^{d}\right)$. Then

$$
\left|\int_{0}^{t} a\left(B_{s}\right) \cdot d B_{s}+\frac{1}{2} \int_{0}^{t} \nabla \cdot a\left(B_{s}\right) d s\right|<\infty \quad \text { almost surely. }
$$


Proof. (1) is standard. To see (2) take $f \in\left(L_{\mathrm{loc}}^{2}\left(\mathbb{R}^{d}\right)\right)^{d}$, then

$$
\mathbb{E}_{P}^{x}\left[\int_{0}^{t} \chi_{\xi}\left(B_{s}\right)\left|f\left(B_{s}\right)\right|^{2} d s\right]<\infty, \quad \xi>0
$$

for any indicator function $\chi_{\xi}$ of the set $\prod_{\mu=1}^{d}[-\xi, \xi]$. Hence $\int_{0}^{t} \chi_{\xi}\left(B_{s}\right)\left|f\left(B_{s}\right)\right|^{2} d s<\infty$ for almost all $\omega$. For each $\omega$ there exists $b(\omega)$ such that $\sup _{0 \leq s \leq t}\left|B_{s}(\omega)\right|<b(\omega)$. Take $\xi=\xi(\omega)$ such that $\xi>b(\omega)$. Then $\int_{0}^{t}\left|f\left(B_{s}(\omega)\right)\right|^{2} d s=\int_{0}^{t} \chi_{\xi}\left(B_{s}(\omega)\right)\left|f\left(B_{s}(\omega)\right)\right|^{2} d s<$ $\infty$, implying $P^{x}\left(\int_{0}^{t}\left|f\left(B_{s}\right)\right|^{2} d s<\infty\right)=1$, thus (2) follows. To see (3), note that

$$
\mathbb{E}_{P}^{x}\left[\left|\int_{0}^{t} \chi_{\xi}\left(B_{s}\right) \nabla \cdot a\left(B_{s}\right) d s\right|\right] \leq \int_{0}^{t} d s \int_{\mathbb{R}^{d}} d y \chi_{\xi}(s y)|(\nabla \cdot a)(s y)| \frac{t e^{-|y|^{2} / 2}}{(2 \pi)^{d / 2}}<\infty
$$

for any indicator function $\chi_{\xi}$, whence follows that $\left|\int_{0}^{t} \nabla \cdot a\left(B_{s}\right) d s\right|<\infty$ for almost every $\omega$. Thus (3) is obtained.

qed

For $a \in\left(L_{\mathrm{loc}}^{2}\left(\mathbb{R}^{d}\right)\right)^{d}$ such that $\nabla \cdot a \in L_{\mathrm{loc}}^{1}\left(\mathbb{R}^{d}\right)$, we denote

$$
\int_{0}^{t} a\left(B_{s}\right) \circ d B_{s}=\int_{0}^{t} a\left(B_{s}\right) \cdot d B_{s}+\frac{1}{2} \int_{0}^{t} \nabla \cdot a\left(B_{s}\right) d s .
$$

Proposition 3.7 Under Assumption (A2) we have

$$
\left(f, e^{-t h} g\right)=\int_{\mathbb{R}^{d}} d x \mathbb{E}_{P}^{x}\left[\overline{f\left(B_{0}\right)} g\left(B_{t}\right) e^{-i \int_{0}^{t} a\left(B_{s}\right) \circ d B_{s}}\right]
$$

Proof. Equality (3.12) is well known as the Feynman-Kac-Itô formula, which in [Sim04, Theorem 15.5] was shown for $a \in L_{\text {loc }}^{2}\left(\mathbb{R}^{d}\right)$, however, with $\nabla \cdot a=0$. We provide a proof of (3.12) under Assumption (A2) for a self-contained presentation.

By using a mollifier we can take a sequence $a_{n} \in\left(C_{0}^{\infty}\left(\mathbb{R}^{d}\right)\right)^{d}, n=1,2, \ldots$, such that $a_{n} \rightarrow a$ in $\left(L_{\mathrm{loc}}^{2}\right)^{d}$ and $\nabla \cdot a_{n} \rightarrow \nabla \cdot a$ in $L_{\mathrm{loc}}^{1}$ as $n \rightarrow \infty$. Let $\chi_{R}=\chi\left(x^{1} / R\right) \cdots \chi\left(x^{d} / R\right)$, $R \in \mathbb{N}$, where $\chi \in C_{0}^{\infty}(\mathbb{R})$ such that $0 \leq \chi \leq 1, \chi(x)=1$ for $|x|<1$ and $\chi(x)=0$ for $|x| \geq 2$. Denote $h=h(a)$. Since $\chi_{R} a_{n} \rightarrow \chi_{R} a$ as $n \rightarrow \infty$ in $\left(L_{\text {loc }}^{2}\right)^{d}$ and $\chi_{R} a \rightarrow a$ as $R \rightarrow \infty$ in $\left(L_{\mathrm{loc}}^{2}\right)^{d}$, it follows [LS81, Lemma $\left.5(3.17)\right]$ that $e^{-t h\left(\chi_{R} a_{n}\right)} \rightarrow e^{-t h\left(\chi_{R} a\right)}$ as $n \rightarrow \infty$ and $e^{-t h\left(\chi_{R} a\right)} \rightarrow e^{-t h(a)}$ as $R \rightarrow \infty$ in strong sense. Furthermore, (3.12) remains true for $a$ replaced by $\chi_{R} a_{n} \in\left(C_{0}^{\infty}\left(\mathbb{R}^{d}\right)\right)^{d}$.

Since $\chi_{R} a_{n} \in\left(C_{0}^{\infty}\left(\mathbb{R}^{d}\right)\right)^{d}$ and $\chi_{R} a_{n} \rightarrow \chi_{R} a$ in $\left(L^{2}\right)^{d}$ as $n \rightarrow \infty$, it follows that

$$
\int_{0}^{t} \chi_{R}\left(B_{s}\right) a_{n}\left(B_{s}\right) \cdot d B_{s} \rightarrow \int_{0}^{t} \chi_{R}\left(B_{s}\right) a\left(B_{s}\right) \cdot d B_{s}
$$


almost surely and since $\nabla \cdot\left(\chi_{R} a_{n}\right)=\left(\nabla \chi_{R}\right) \cdot a_{n}+\chi_{R}\left(\nabla \cdot a_{n}\right) \rightarrow\left(\nabla \chi_{R}\right) \cdot a+\chi_{R}(\nabla \cdot a)$ in $L^{1}\left(\mathbb{R}^{d}\right)$, it furthermore follows that

$$
\int_{0}^{t} \nabla \cdot\left(\chi_{R}\left(B_{s}\right) a_{n}\left(B_{s}\right)\right) d s \rightarrow \int_{0}^{t}\left(\nabla \chi_{R}\left(B_{s}\right)\right) \cdot a\left(B_{s}\right) d s+\chi_{R}\left(B_{s}\right)\left(\nabla \cdot a\left(B_{s}\right)\right) d s
$$

strongly in $L^{1}\left(\Omega_{P}, d P^{x}\right)$. Thus there exists a subsequence $n^{\prime}$ such that (3.13) and (3.14) with $n$ replaced by $n^{\prime}$ hold almost surely. Hence (3.12) results by a limiting argument for $a$ replaced by $\chi_{R} a$. Let

$$
\begin{aligned}
& \Omega_{+}(R)=\left\{\omega \in \Omega_{P} \mid \max _{0 \leq s \leq t, 1 \leq \mu \leq d} B_{s}^{\mu}(\omega) \leq R\right\} \\
& \Omega_{-}(R)=\left\{\omega \in \Omega_{P} \mid \min _{0 \leq s \leq t, 1 \leq \mu \leq d} B_{s}^{\mu}(\omega) \geq-R\right\}
\end{aligned}
$$

and

$$
I(R)=\left|\int_{0}^{t} \chi_{R}\left(B_{s}\right) a\left(B_{s}\right) \cdot d B_{s}-\int_{0}^{t} a\left(B_{s}\right) \cdot d B_{s}\right| .
$$

We show that $I(R) \rightarrow 0$ in probability as $R \rightarrow \infty$. Note that the random variables $\max _{0 \leq s \leq t} B_{s}^{\mu}(\omega)$ and $\min _{0 \leq s \leq t} B_{s}^{\mu}(\omega)$ have the same distribution and

$$
P\left(\Omega_{-}(R)\right)=P\left(\Omega_{+}(R)\right)=\prod_{\mu=1}^{d} P\left(\left|B_{t}^{\mu}\right| \leq R\right)=\left(\frac{2}{\sqrt{2 \pi t}} \int_{0}^{R} e^{-y^{2} /(2 t)} d y\right)^{d} .
$$

Since $\chi_{R}\left(B_{s}\right)=1$ for all $0 \leq s \leq t$ on $\Omega_{+}(R) \cap \Omega_{-}(R), I(R)=0$ on $\Omega_{+}(R) \cap \Omega_{-}(R)$, we have

$$
P(I(R) \geq \epsilon)=P\left(I(R) \geq \epsilon, \Omega_{+}(R)^{c} \cup \Omega_{-}(R)^{c}\right) \leq 2\left(\frac{2}{\sqrt{2 \pi t}} \int_{R}^{\infty} e^{-y^{2} /(2 t)} d y\right)^{d} .
$$

Hence $\lim _{R \rightarrow \infty} P(I(R) \geq \epsilon)=0$. Thus there exists a subsequence $R^{\prime}$ such that $\int_{0}^{t} \chi_{R^{\prime}}\left(B_{s}\right) a\left(B_{s}\right) \cdot d B_{s} \rightarrow \int_{0}^{t} a\left(B_{s}\right) \cdot d B_{s}$ almost surely as $R^{\prime} \rightarrow \infty$. In a similar way it is seen that $\int_{0}^{t} \chi_{R^{\prime \prime}}\left(B_{s}\right) \nabla \cdot a\left(B_{s}\right) d s \rightarrow \int_{0}^{t} \nabla \cdot a\left(B_{s}\right) d s$ as $R^{\prime \prime} \rightarrow \infty$ almost surely for some subsequence $R^{\prime \prime}$ of $R^{\prime}$. Moreover,

$$
\int_{0}^{t} \nabla \chi_{R}\left(B_{s}\right) \cdot a\left(B_{s}\right) d s=\frac{1}{R} \int_{0}^{t} \nabla \chi\left(B_{s} / R\right) \cdot a\left(B_{s}\right) d s \rightarrow 0
$$

in probability, and then for some subsequence $R^{\prime \prime \prime}$ of $R^{\prime \prime}$, (3.15) converges to zero almost surely. Thus $\int_{0}^{t} \chi_{R^{\prime \prime \prime}}\left(B_{s}\right) a\left(B_{s}\right) \circ d B_{s} \rightarrow \int_{0}^{t} a\left(B_{s}\right) \circ d B_{s}$ almost surely, and (3.12) holds for any a satisfying Assumption (A2). 


\subsection{Functional integral representation}

Now we turn to constructing a functional integral representation for generalized Schrödinger operators including a vector potential term defined by (3.5).

A key element in our construction of a Feynman-Kac-type formula for $e^{-t H^{\Psi}}$ is to make use of a Lévy subordinator.

Theorem 3.8 Let $\Psi \in \mathscr{B}_{0}$ and $V \in L^{\infty}\left(\mathbb{R}^{d}\right)$. Under Assumption (A2) we have

$$
\left(f, e^{-t H^{\Psi}} g\right)=\int_{\mathbb{R}^{d}} d x \mathbb{E}_{P \times \nu}^{x, 0}\left[\overline{f\left(B_{0}\right)} g\left(B_{T_{t}^{\Psi}}\right) e^{-i \int_{0}^{T_{t}^{\Psi}} a\left(B_{s}\right) \circ d B_{s}} e^{-\int_{0}^{t} V\left(B_{T_{s}^{\Psi}}\right) d s}\right]
$$

Proof. We divide the proof into four steps. To simplify the notation, in this proof we drop the superscript $\Psi$ of the subordinator.

(Step 1) Suppose $V=0$. Then we claim that

$$
\left(f, e^{-t \Psi(h)} g\right)=\int_{\mathbb{R}^{d}} d x \mathbb{E}_{P \times \nu}^{x, 0}\left[\overline{f\left(B_{0}\right)} g\left(B_{T_{t}}\right) e^{-i \int_{0}^{T_{t}} a\left(B_{s}\right) \circ d B_{s}}\right] .
$$

To prove (3.17) let $E^{h}$ denote the spectral projection of the self-adjoint operator $h$. Then

$$
\left(f, e^{-t \Psi(h)} g\right)=\int_{\operatorname{Spec}(h)} e^{-t \Psi(u)} d\left(f, E_{u}^{h} g\right) .
$$

By inserting identity (2.2) in (3.18) we obtain

$$
\left(f, e^{-t \Psi(h)} g\right)=\int_{\operatorname{Spec}(h)} \mathbb{E}_{\nu}^{0}\left[e^{-T_{t} u}\right] d\left(f, E_{u}^{h} g\right)=\mathbb{E}_{\nu}^{0}\left[\left(f, e^{-T_{t} h} g\right)\right] .
$$

Then by the Feynman-Kac-Itô formula for $e^{-t h}$ we have

$$
\left(f, e^{-t \Psi(h)} g\right)=\mathbb{E}_{\nu}^{0}\left[\int_{\mathbb{R}^{d}} d x \mathbb{E}_{P}^{x}\left[\overline{f\left(B_{0}\right)} g\left(B_{T_{t}}\right) e^{-i \int_{0}^{T_{t}} a\left(B_{s}\right) \circ d B_{s}}\right]\right]
$$

thus (3.17) follows.

(Step 2) Let $0=t_{0}<t_{1}<\cdots<t_{n}, f_{0}, f_{n} \in L^{2}\left(\mathbb{R}^{d}\right)$ and assume that $f_{j} \in L^{\infty}\left(\mathbb{R}^{d}\right)$ for $j=1, \ldots, n-1$. We claim that

$$
\left(f_{0}, \prod_{j=1}^{n} e^{-\left(t_{j}-t_{j-1}\right) \Psi(h)} f_{j}\right)=\int_{\mathbb{R}^{d}} d x \mathbb{E}_{P \times \nu}^{x, 0}\left[\overline{f_{0}\left(B_{0}\right)}\left(\prod_{j=1}^{n} f_{j}\left(B_{T_{t_{j}}}\right)\right) e^{-i \int_{0}^{T_{t}} a\left(B_{s}\right) \circ d B_{s}}\right] .
$$


For easing the notation write $G_{j}(\cdot)=f_{j}(\cdot)\left(\prod_{i=j+1}^{n} e^{-\left(t_{i}-t_{i-1}\right) \Psi(h)} f_{i}\right)(\cdot)$. By (Step 1) the left hand side of (3.19) can be represented as

$$
\int_{\mathbb{R}^{d}} d x \mathbb{E}_{P \times \nu}^{x, 0}\left[\overline{f_{0}\left(B_{0}\right)} e^{-i \int_{0}^{T_{t_{1}}-t_{0}} a\left(B_{s}\right) \circ d B_{s}} G_{1}\left(B_{T_{t_{1}-t_{0}}}\right)\right]
$$

Let $\mathscr{F}_{t}^{\mathrm{P}}=\sigma\left(B_{s}, 0 \leq s \leq t\right)$ and $\mathscr{F}_{t}^{\nu}=\sigma\left(T_{s}, 0 \leq s \leq t\right)$ be the natural filtrations. An application of the Markov property of $B_{t}$ yields

$$
\begin{aligned}
& \left(f_{0}, \prod_{j=1}^{n} e^{-\left(t_{j}-t_{j-1}\right) \Psi(h)} f_{j}\right) \\
& =\int_{\mathbb{R}^{d}} d x \mathbb{E}_{P \times \nu}^{x, 0}\left[\overline{f_{0}\left(B_{0}\right)} e^{-i \int_{0}^{T_{t_{1}}} a\left(B_{s}\right) \circ d B_{s}} \mathbb{E}_{\nu}^{0} \mathbb{E}_{P}^{B_{T_{t_{1}}}}\left[f_{1}\left(B_{0}\right) e^{-i \int_{0}^{T_{t_{2}}-t_{1}} a\left(B_{s}\right) \circ d B_{s}} G_{2}\left(B_{T_{t_{2}-t_{1}}}\right)\right]\right] \\
& =\int_{\mathbb{R}^{d}} d x \mathbb{E}_{P \times \nu}^{x, 0}\left[\overline{f_{0}\left(B_{0}\right)} e^{-i \int_{0}^{T_{t_{1}}} a\left(B_{s}\right) \circ d B_{s}}\right.
\end{aligned}
$$

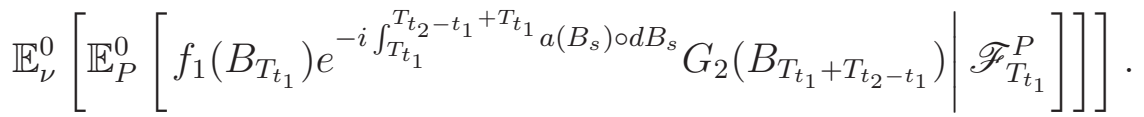

Hence we obtain

$$
\begin{aligned}
& \left(f_{0}, \prod_{j=1}^{n} e^{-\left(t_{j}-t_{j-1}\right) \Psi(h)} f_{j}\right) \\
& =\int_{\mathbb{R}^{d}} d x \mathbb{E}_{P \times \nu}^{x, 0}\left[\overline{f_{0}\left(B_{0}\right)} e^{-i \int_{0}^{T_{t_{1}}} a\left(B_{s}\right) \circ d B_{s}} \mathbb{E}_{\nu}^{0}\left[f_{1}\left(B_{T_{t_{1}}}\right) e^{-i \int_{T_{t_{1}}}^{T_{t_{2}}-t_{1}+T_{t_{1}}} a\left(B_{s}\right) \circ d B_{s}} G_{2}\left(B_{T_{t_{1}}+T_{t_{2}-t_{1}}}\right)\right]\right] .
\end{aligned}
$$

The right hand side above can be rewritten as

$$
\int_{\mathbb{R}^{d}} d x \mathbb{E}_{P \times \nu}^{x, 0}\left[\overline{f_{0}\left(B_{0}\right)} e^{-i \int_{0}^{T_{t_{1}}} a\left(B_{s}\right) \circ d B_{s}} f_{1}\left(B_{T_{t_{1}}}\right) \mathbb{E}_{\nu}^{T_{t_{1}}}\left[e^{-i \int_{0}^{T_{t_{2}}-t_{1}} a\left(B_{s}\right) \circ d B_{s}} G_{2}\left(B_{T_{t_{2}-t_{1}}}\right)\right]\right] .
$$

Using now the Markov property of $T_{t}$ we see that

$$
\begin{aligned}
& \left(f_{0}, \prod_{j=1}^{n} e^{-\left(t_{j}-t_{j-1}\right) \Psi(h)} f_{j}\right) \\
& \quad=\int_{\mathbb{R}^{d}} d x \mathbb{E}_{P \times \nu}^{x, 0}\left[\overline { f _ { 0 } ( B _ { 0 } ) } e ^ { - i \int _ { 0 } ^ { T _ { t _ { 1 } } } a ( B _ { s } ) \circ d B _ { s } } f _ { 1 } ( B _ { T _ { t _ { 1 } } } ) \mathbb { E } _ { \nu } ^ { 0 } \left[e^{\left.\left.-i \int_{T_{t_{1}}}^{T_{t_{2}} a\left(B_{s}\right) \circ d B_{s}} G_{2}\left(B_{T_{t_{2}}}\right) \mid \mathscr{F}_{t_{1}}^{\nu}\right]\right]}\right.\right. \\
& \quad=\int_{\mathbb{R}^{d}} d x \mathbb{E}_{P \times \nu}^{x, 0}\left[\overline{f_{0}\left(B_{0}\right)} e^{-i \int_{0}^{T_{t_{1}}} a\left(B_{s}\right) \circ d B_{s}} f_{1}\left(B_{T_{t_{1}}}\right) e^{-i \int_{T_{t_{1}}}^{T_{t_{2}}} a\left(B_{s}\right) \circ d B_{s}} G_{2}\left(B_{T_{t_{2}}}\right)\right] .
\end{aligned}
$$

By the above procedure we obtain (3.19). 
(Step 3) Suppose now that $0 \neq V \in L^{\infty}$ and it is continuous; we prove (3.16) for such $V$. Since $H^{\Psi}$ is self-adjoint on $D(\Psi(h)) \cap D(V)$ the Trotter product formula holds:

$$
\left(f, e^{-t H^{\Psi}} g\right)=\lim _{n \rightarrow \infty}\left(f,\left(e^{-(t / n) \Psi(h)} e^{-(t / n) V}\right)^{n} g\right) .
$$

(Step 2) yields

$$
\begin{aligned}
\left(f, e^{-t H^{\Psi}} g\right) & =\lim _{n \rightarrow \infty} \int_{\mathbb{R}^{d}} d x \mathbb{E}_{P \times \nu}^{x, 0}\left[\overline{f\left(B_{0}\right)} g\left(B_{T_{t}}\right) e^{-i \int_{0}^{T_{t}} a\left(B_{s}\right) \circ d B_{s}} e^{-\sum_{j=1}^{n}(t / n) V\left(B_{T_{t j} / n}\right)}\right] \\
& =\text { r.h.s. (3.19) }
\end{aligned}
$$

Here we used that since $s \mapsto B_{T_{s}(\tau)}(\omega)$ has càdlàg paths, $V\left(B_{T_{s}(\tau)}(\omega)\right)$ is continuous in $s \in[0, t]$ for each $(\omega, \tau)$ except for at most finite points. Therefore $\sum_{j=1}^{n} \frac{t}{n} V\left(B_{T_{t j / n}}\right) \rightarrow$ $\int_{0}^{t} V\left(B_{T_{s}}\right) d s$ as $n \rightarrow \infty$ for each path and exists as a Riemann integral.

(Step 4) An application of the method in [Sim04, Theorem 6.2] will complete the proof of Theorem 3.8. To do that, suppose that $V \in L^{\infty}$ and $V_{n}=\phi(x / n)\left(V * j_{n}\right)$, where $j_{n}=n^{d} \phi(x n)$ with $\phi \in C_{0}^{\infty}\left(\mathbb{R}^{d}\right)$ such that $0 \leq \phi \leq 1, \int \phi(x) d x=1$ and $\phi(0)=1$. Then $V_{n}(x) \rightarrow V(x)$ almost everywhere. $V_{n}$ is bounded and continuous, moreover $V_{n}(x) \rightarrow V(x)$ as $n \rightarrow \infty$ for $x \notin \mathscr{N}$, where the Lebesgue measure of $\mathscr{N}$ is zero. Thus for almost every $(\omega, \tau) \in \Omega_{P} \times \Omega_{N}$, the measure of $\left\{t \in[0, \infty) \mid B_{T_{t}(\tau)}(\omega) \in \mathscr{N}\right\}$ is zero. Hence $\int_{0}^{t} V_{n}\left(B_{T_{s}}\right) d s \rightarrow \int_{0}^{t} V\left(B_{T_{s}}\right) d s$ as $n \rightarrow \infty$ almost surely under $P^{x} \times \nu^{0}$,

$$
\begin{aligned}
& \int_{\mathbb{R}^{d}} d x \mathbb{E}_{P \times \nu}^{x, 0}\left[\overline{f\left(B_{0}\right)} g\left(B_{T_{t}}\right) e^{-i \int_{0}^{T_{t}} a\left(B_{s}\right) \circ d B_{s}} e^{-\int_{0}^{t} V_{n}\left(B_{T_{s}}\right) d s}\right] \\
& \rightarrow \int_{\mathbb{R}^{d}} d x \mathbb{E}_{P \times \nu}^{x, 0}\left[\overline{f\left(B_{0}\right)} g\left(B_{T_{t}}\right) e^{-i \int_{0}^{T_{t}} a\left(B_{s}\right) \circ d B_{s}} e^{-\int_{0}^{t} V\left(B_{T_{s}}\right) d s}\right]
\end{aligned}
$$

as $n \rightarrow \infty$. On the other hand, $e^{-t\left(\Psi(h)+V_{n}\right)} \rightarrow e^{-t(\Psi(h)+V)}$ strongly as $n \rightarrow \infty$, since $\Psi(h)+V_{n}$ converges to $\Psi(h)+V$ on the common domain $D(\Psi(h))$. Thus the theorem follows.

qed

Setting $a=0$ and $\Psi(u)=u^{\alpha}$ yields an interesting class of its own.

Definition 3.9 (Fractional Schrödinger operator) Let $0<\alpha<1$ and $\Psi(u)=$ $u^{\alpha}$. We call

$$
H_{\alpha}=\left(\frac{1}{2} \mathrm{p}^{2}\right)^{\alpha}+V
$$

fractional Schrödinger operator with exponent $\alpha$. 


\section{Corollary 3.10 (Functional integral for fractional Schrödinger operator)}

Let $T_{t}^{\Psi}$ be the subordinator for a fractional Schrödinger operator, i.e., an $\alpha$-stable process, and $V \in L^{\infty}\left(\mathbb{R}^{d}\right)$. Then

$$
\left(f, e^{-t H_{\alpha}} g\right)=\int_{\mathbb{R}^{d}} d x \mathbb{E}_{P \times \nu}^{x, 0}\left[\overline{f\left(B_{0}\right)} g\left(B_{T_{t}}\right) e^{-\int_{0}^{t} V\left(B_{T_{s}^{\Psi}}\right) d s}\right] .
$$

We use the notation $\mathscr{E}_{T}=\inf \operatorname{Spec} T$ here and in Sections 5 and 6 below.

Corollary 3.11 (Diamagnetic inequality) Let $\Psi \in \mathscr{B}_{0}, V \in L^{\infty}\left(\mathbb{R}^{d}\right)$, and Assumption (A2) hold. Then

$$
\left|\left(f, e^{-t H^{\Psi}} g\right)\right| \leq\left(|f|, e^{-t\left(\Psi\left(\mathrm{p}^{2} / 2\right)+V\right)}|g|\right)
$$

and the energy comparison inequality

$$
\mathscr{E}_{\Psi\left(\mathrm{p}^{2} / 2\right)+V} \leq \mathscr{E}_{H^{\Psi}}
$$

holds.

Proof. By Theorem 3.8 we have

$$
\left|\left(f, e^{-t H^{\Psi}} g\right)\right| \leq \int_{\mathbb{R}^{d}} d x \mathbb{E}_{P \times \nu}^{x, 0}\left[\left|f\left(B_{0}\right)\right|\left|g\left(B_{T_{t}^{\Psi}}\right)\right| e^{-\int_{0}^{t} V\left(B_{T_{s}^{\Psi}}\right) d s}\right] .
$$

The right hand side above coincides with that of (3.21), and (3.22) follows directly from (3.21).

qed

\subsection{Singular external potentials}

By making use of the functional integral representation obtained in the previous subsection we can now also consider more singular external potentials.

Theorem 3.12 Let Assumption (A2) hold.

(1) Suppose $|V|$ is relatively form bounded with respect to $\Psi\left(\mathrm{p}^{2} / 2\right)$ with relative bound $b$. Then $|V|$ is also relatively form bounded with respect to $\Psi(h)$ with a relative bound not larger than $b$.

(2) Suppose $|V|$ is relatively bounded with respect to $\Psi\left(\mathrm{p}^{2} / 2\right)$ with relative bound $b$. Then $|V|$ is also relatively bounded with respect to $\Psi(h)$ with a relative bound not larger than $b$. 
Proof. The proof is parallel with that of [Sim04, Theorem 15.6]. By virtue of Corollary 3.11 we have

$$
\left|\left(f, e^{-t \Psi(h)} g\right)\right| \leq\left(|f|, e^{-t \Psi\left(\mathrm{p}^{2} / 2\right)}|g|\right) .
$$

Since $(\Psi(h)+E)^{-1 / 2}=\frac{1}{\sqrt{\pi}} \int_{0}^{\infty} t^{-1 / 2} e^{-t(\Psi(h)+E)} d t, E>0$, (3.23) implies that

$$
\left|(\Psi(h)+E)^{-1 / 2} f\right|(x) \leq\left(\Psi\left(\mathrm{p}^{2} / 2\right)+E\right)^{-1 / 2}|f|(x)
$$

for almost every $x \in \mathbb{R}^{d}$. Hence we have

$$
\left.|V(x)|^{1 / 2}\left|(\Psi(h)+E)^{-1 / 2} f\right|(x) \leq|V(x)|^{1 / 2} \Psi\left(\mathrm{p}^{2} / 2\right)+E\right)^{-1 / 2}|f|(x)
$$

and

$$
\frac{\left\||V|^{1 / 2}(\Psi(h)+E)^{-1 / 2} f\right\|}{\|f\|} \leq \frac{\left\||V|^{1 / 2}\left(\Psi\left(\mathrm{p}^{2} / 2\right)+E\right)^{-1 / 2}|f|\right\|}{\|f\|} .
$$

Similarly, by using $(\Psi(h)+E)^{-1}=\int_{0}^{\infty} e^{-t(\Psi(h)+E)} d t, E>0$, we have

$$
\frac{\left\|V \mid(\Psi(h)+E)^{-1} f\right\|}{\|f\|} \leq \frac{\left\||V|\left(\Psi\left(\mathrm{p}^{2} / 2\right)+E\right)^{-1}|f|\right\|}{\|f\|} .
$$

On taking the limit $E \rightarrow \infty$, the right hand sides of (3.25) and (3.26) converge to $b$; compare [HS95, Lemma 13.6], Sim04, AHS78. Hence (1) follows by (3.25) and (2) by (3.26).

qed

Corollary 3.13 (1) Take Assumption (A2) and let $V$ be relatively bounded with respect to $\Psi\left(\mathrm{p}^{2} / 2\right)$ with relative bound strictly smaller than one. Then $\Psi(h)+V$ is self-adjoint on $D(\Psi(h))$ and bounded from below. Moreover, it is essentially self-adjoint on any core of $\Psi(h)$. (2) Suppose furthermore (A3). Then $C_{0}^{\infty}\left(\mathbb{R}^{d}\right)$ is an operator core of $\Psi(h)+V$.

Proof. (1) By (2) of Theorem 3.12, $V$ is relatively bounded with respect to $\Psi(h)$ with a relative bound strictly smaller than one. Then the corollary follows by the Kato-Rellich theorem. (2) follows from Theorem 3.3.

Theorem 3.12 also allows $\Psi(h)+V$ to be defined in form sense. Let $V=V_{+}-V_{-}$ where $V_{+}=\max \{V, 0\}$ and $V_{-}=\min \{-V, 0\}$. Theorem 3.12 implies that whenever $V_{-}$is form bounded to $\Psi\left(\mathrm{p}^{2} / 2\right)$ with a relative bound strictly smaller than one, it is also form bounded with respect to $\Psi(h)$ with a relative bound strictly smaller than 
one. Moreover, assume that $V_{+} \in L_{\mathrm{loc}}^{1}\left(\mathbb{R}^{d}\right)$. We see that given Assumption (A1), $Q(\Psi(h)) \cap Q\left(V_{+}\right) \supset C_{0}^{\infty}\left(\mathbb{R}^{d}\right)$ by Corollary 3.13, In particular, $Q(\Psi(h)) \cap Q\left(V_{+}\right)$is dense. Define the quadratic form

$$
\mathrm{q}(f, f):=\left(\Psi(h)^{1 / 2} f, \Psi(h)^{1 / 2} f\right)+\left(V_{+}^{1 / 2} f, V_{+}^{1 / 2} f\right)-\left(V_{-}^{1 / 2} f, V_{-}^{1 / 2} f\right)
$$

on $Q(\Psi(h)) \cap Q\left(V_{+}\right)$. By the KLMN Theorem [RS78] q is a semibounded closed form.

Definition 3.14 (Generalized Schrödinger operator with singular $V$ ) Let Assumption (A2) hold and $V=V_{+}-V_{-}$be such that $V_{+} \in L_{\text {loc }}^{1}\left(\mathbb{R}^{d}\right)$ and $V_{-}$is form bounded with respect to $\Psi\left(\frac{1}{2} \mathrm{p}^{2}\right)$ with a relative bound strictly less than 1 . We denote the self-adjoint operator associated with (3.27) by $\Psi(h) \dot{+} V_{+} \dot{-} V_{-}$defined as a quadratic form sum.

Since we need (A2) to show the relative form boundedness of $V_{-}$with respect to $\Psi(h)$, (A2) is assumed in Definition (3.14).

Now we are in the position to extend Theorem 3.8 to potentials expressed as form sums.

Theorem 3.15 Take Assumption (A2). Let $V=V_{+}-V_{-}$be such that $V_{+} \in L_{\text {loc }}^{1}\left(\mathbb{R}^{d}\right)$ and $V_{-}$is infinitesimally small with respect to $\Psi\left(\frac{1}{2} \mathrm{p}^{2}\right)$ in form sense. Then the functional integral representation given by Theorem 3.8 also holds for $\Psi(h) \dot{+} V_{+} \dot{-} V_{-}$.

Proof. Write

$$
V_{+, n}(x)=\left\{\begin{array}{ll}
V_{+}(x), & V_{+}(x)<n, \\
n, & V_{+}(x) \geq n,
\end{array} \quad V_{-, m}(x)= \begin{cases}V_{-}(x), & V_{-}(x)<m, \\
m, & V_{-}(x) \geq m .\end{cases}\right.
$$

For simplicity we write just $\Psi$ for $\Psi(h)$. Define the closed quadratic forms

$$
\begin{aligned}
\mathrm{q}_{n, m}(f, f) & =\left(\Psi^{1 / 2} f, \Psi^{1 / 2} f\right)+\left(V_{+, n}^{1 / 2} f, V_{+, n}^{1 / 2} f\right)-\left(V_{-, m}^{1 / 2} f, V_{-, m}^{1 / 2} f\right), \\
\mathrm{q}_{n, \infty}(f, f) & =\left(\Psi^{1 / 2} f, \Psi^{1 / 2} f\right)+\left(V_{+, n}^{1 / 2} f, V_{+, n}^{1 / 2} f\right)-\left(V_{-}^{1 / 2} f, V_{-}^{1 / 2} f\right), \\
\mathrm{q}_{\infty, \infty}(f, f) & =\left(\Psi^{1 / 2} f, \Psi^{1 / 2} f\right)+\left(V_{+}^{1 / 2} f, V_{+}^{1 / 2} f\right)-\left(V_{-}^{1 / 2} f, V_{-}^{1 / 2} f\right),
\end{aligned}
$$

where the form domains are given by

$$
Q\left(\mathrm{q}_{n, m}\right)=Q(\Psi), \quad Q\left(\mathrm{q}_{n, \infty}\right)=Q(\Psi), \quad Q\left(\mathrm{q}_{\infty, \infty}\right)=Q(\Psi) \cap Q\left(V_{+}\right) .
$$

Clearly, $\mathrm{q}_{n, m} \geq \mathrm{q}_{n, m+1} \geq \mathrm{q}_{n, m+2} \geq \ldots \geq \mathrm{q}_{n, \infty}$ and $\mathrm{q}_{n, m} \rightarrow \mathrm{q}_{n, \infty}$ in the sense of quadratic forms on $\cup_{m} Q\left(\mathrm{q}_{n, m}\right)=Q(\Psi)$. Since $\mathrm{q}_{n, \infty}$ is closed on $Q(\Psi)$, by the monotone convergence theorem for a non-increasing sequence of forms (see Kat76, Theorem 
VIII.3.11] and [Sim78, Theorem 3.2]) the associated positive self-adjoint operators satisfy $\Psi \dot{+} V_{+, n} \dot{-} V_{-, m} \rightarrow \Psi \dot{+} V_{+, n} \dot{-} V_{-}$in strong resolvent sense, which implies that

$$
e^{-t\left(\Psi \dot{+} V_{+, n} \dot{-} V_{-, m}\right)} \rightarrow e^{-t\left(\Psi \dot{+} V_{+, n} \dot{-} V_{-}\right)}
$$

strongly as $m \rightarrow \infty$, for all $t \geq 0$. Similarly, we have $\mathrm{q}_{n, \infty} \leq \mathrm{q}_{n+1, \infty} \leq \mathrm{q}_{n+2, \infty} \leq \ldots \leq$ $\mathrm{q}_{\infty, \infty}$ and $\mathrm{q}_{n, \infty} \rightarrow \mathrm{q}_{\infty, \infty}$ in quadratic form sense on $\left\{f \in \cap_{n} Q\left(\mathrm{q}_{n, \infty}\right) \mid \sup _{n} \mathrm{q}_{n, \infty}(f, f)<\right.$ $\infty\}=Q(\Psi) \cap Q\left(V_{+}\right)$. Hence by the monotone convergence theorem for a non-decreasing sequence of forms (see [Sim78, Theorem 3.1 and Theorem 4,1] and [Kat76, Theorem VIII.3.13 with Supplementary notes to Chapter VIII,5 (p.575)]) we obtain

$$
e^{-t\left(\Psi \dot{+} V_{+, n} \dot{-} V_{-}\right)} \rightarrow e^{-t\left(\Psi \dot{+} V_{+} \dot{-} V_{-}\right)},
$$

for all $t \geq 0$, in strong sense as $n \rightarrow \infty$.

On the other hand, we look at the convergence of the expression

$$
\int d x \mathbb{E}_{P \times \nu}^{x, 0}\left[e^{-\int_{0}^{t}\left(V_{+, n}-V_{-, m}\right)\left(B_{T_{s}^{\Psi}}\right) d s} I\right] .
$$

Here $I=\overline{f\left(B_{0}\right)} e^{-i \int_{0}^{T_{t}} a\left(B_{s}\right) \circ d B_{s}} g\left(B_{T_{t}}\right)$. Decompose $I$ into its real and imaginary parts, and further into their positive and negative parts $\Re I=\Re I_{+}-\Re I_{-}$and $\Im I=\Im I_{+}-\Im I_{-}$. Then by (3.28) and the monotone convergence theorem

$$
\int d x \mathbb{E}_{P \times \nu}^{x, 0}\left[e^{-\int_{0}^{t}\left(V_{+, n}-V_{-, m}\right)\left(B_{T_{s}^{\Psi}}\right) d s} \Re I_{+}\right] \longrightarrow \int d x \mathbb{E}_{P \times \nu}^{x, 0}\left[e^{-\int_{0}^{t}\left(V_{+, n}-V_{-}\right)\left(B_{T_{s}^{\Psi}}\right) d s} \Re I_{+}\right]
$$

as $m \rightarrow \infty$. Similarly, the remaining three terms $\Re I_{-}, \Im I_{+}$and $\Im I_{-}$also converge. Thus (3.30) converges to $\int d x \mathbb{E}_{P \times \nu}^{x, 0}\left[e^{-\int_{0}^{t}\left(V_{+, n}-V_{-}\right)\left(B_{T_{s}^{\Psi}}\right) d s} I\right]$ as $m \rightarrow \infty$. Moreover,

$$
\int d x \mathbb{E}_{P \times \nu}^{x, 0}\left[e^{-\int_{0}^{t}\left(V_{+, n}-V_{-}\right)\left(B_{T_{s}^{\Psi}}\right) d s} I\right] \longrightarrow \int d x \mathbb{E}_{P \times \nu}^{x, 0}\left[e^{-\int_{0}^{t}\left(V_{+}-V_{-}\right)\left(B_{T_{s}^{\Psi}}\right) d s} I\right]
$$

as $n \rightarrow \infty$, by (3.29) and the dominated convergence theorem. Thus the proof is complete.

qed

\section{$4 \quad \Psi$-Kato class potentials}

\subsection{Definition of $\Psi$-Kato class potentials}

In this section we give a meaning to Kato class for potentials $V$ relative to $\Psi$ and extend generalized Schrödinger operators with vector potential to such $V$. 
It is known that the composition of a Brownian motion and a subordinator yields a Lévy process. Recall that for given $\Psi \in \mathscr{B}_{0}$, the random process

$$
X_{t}: \Omega_{P} \times \Omega_{\nu} \ni(\omega, \tau) \mapsto B_{T_{t}^{\Psi}(\tau)}(\omega)
$$

is called $d$-dimensional subordinated Brownian motion with respect to the subordinator $\left(T_{t}^{\Psi}\right)_{t \geq 0}$. It is a Lévy process whose properties are determined by the pair $(b, \lambda)$ in (2.1). Its characteristic function is

$$
\mathbb{E}_{P \times \nu}^{0,0}\left[e^{i \xi \cdot X_{t}}\right]=e^{-t \Psi(\xi \cdot \xi / 2)}, \quad \xi \in \mathbb{R}^{d} .
$$

Assumption 4.1 Let $\Psi \in \mathscr{B}_{0}$ be such that

$$
\int_{\mathbb{R}^{d}} e^{-t \Psi(\xi \cdot \xi / 2)} d \xi<\infty
$$

for all $t>0$.

Let $\Psi \in \mathscr{B}_{0}$ and $(b, \lambda) \in \mathbb{R}_{+} \times \mathscr{L}$ be its corresponding non-negative drift coefficient and Lévy measure, i.e., $\Psi(u)=b u+\int_{0}^{\infty}\left(1-e^{-u y}\right) \lambda(d y)$. It is clear that if $b>0$, then (4.3) is satisfied. In the case of $b=0$ but $\int_{0}^{1} \lambda(d y)<\infty$, since $\sup _{u \geq 0} \Psi(u)<\infty$, (4.3) is not satisfied. Thus $\Psi$ obeying (4.3) at least satisfies $\int_{0}^{1} \lambda(d y)=\infty$ when $b=0$. In this case we have

$$
\Psi\left(u^{2} / 2\right) \geq \int_{0}^{1}\left(1-e^{-u^{2} y / 2}\right) \lambda(d y) \geq\left(1-e^{-1}\right) \int_{0}^{1}\left(\frac{u^{2} y}{2} \wedge 1\right) \lambda(d y) \geq\left(1-e^{-1}\right) \int_{2 / u^{2}}^{1} \lambda(d y) .
$$

Thus in case $b=0$ and $\int_{0}^{1} \lambda(d y)=\infty$, assuming that there exists $\rho(u)$ such that $\int_{2 / u^{2}}^{1} \lambda(d y) \geq \rho(u)$ and $\int_{\mathbb{R}^{d}} e^{-t \rho(|\xi|)} d \xi<\infty$, we can make sure Assumption 4.1 holds.

Under Assumption 4.1 we define

$$
p_{t}(x)=\frac{1}{(2 \pi)^{d}} \int_{\mathbb{R}^{d}} e^{-i x \cdot \xi} e^{-t \Psi(\xi \cdot \xi / 2)} d \xi
$$

and

$$
\Pi_{\lambda}(x)=\int_{0}^{\infty} e^{-\lambda t} p_{t}(x) d t .
$$

$p_{t}(x)$ denotes the distribution density of $X_{t}$ in (4.1) and $\Pi_{\lambda}(x-y)$ is the integral kernel of the resolvent $\left(\Psi\left(\mathrm{p}^{2} / 2\right)+\lambda\right)^{-1}$ with $\lambda>0$, i.e.,

$$
\left(f,\left(\Psi\left(\mathrm{p}^{2} / 2\right)+\lambda\right)^{-1} g\right)=\int_{\mathbb{R}^{d} \times \mathbb{R}^{d}} \overline{f(x)} g(y) \Pi_{\lambda}(x-y) d x d y .
$$


Clearly, $p_{t}(x)$ and $\Pi_{\lambda}(x)$ are spherically symmetric. For $f \in C_{0}^{\infty}\left(\mathbb{R}^{d}\right)$ it follows that

$$
\mathbb{E}_{P \times \nu}^{0,0}\left[f\left(X_{t}\right)\right]=\int f(x) p_{t}(x) d x .
$$

Hence for non-negative $f \in C_{0}^{\infty}\left(\mathbb{R}^{d}\right)$, the right hand side of (4.5) is non-negative since so is the left hand side. Thus $p_{t}(x) \geq 0$ for almost every $x \in \mathbb{R}^{d}$. By a limiting argument with $f \rightarrow 1$, we can also see that $p_{t} \in L^{1}\left(\mathbb{R}^{d}\right)$ and $\left\|p_{t}\right\|_{L^{1}\left(\mathbb{R}^{d}\right)}=1$ by (4.5).

We moreover compute $\Pi_{\lambda}$ as

$$
\Pi_{\lambda}(x)=(2 \pi)^{-d / 2} \frac{1}{|x|^{(d-1) / 2}} \int_{0}^{\infty} \frac{r^{(d-1) / 2}}{\lambda+\Psi\left(r^{2} / 2\right)} \sqrt{r|x|} J_{(d-2) / 2}(r|x|) d r,
$$

with the Bessel function given by

$$
J_{\nu}(s)=\left(\frac{s}{2}\right)^{\nu} \frac{1}{\sqrt{\pi} \Gamma\left(\nu+\frac{1}{2}\right)} \int_{0}^{\pi} e^{i s \cos \theta}(\sin \theta)^{2 \nu} d \theta=\sum_{n=0}^{\infty} \frac{(-1)^{n}}{n ! \Gamma(n+\nu+1)}\left(\frac{s}{2}\right)^{2 n+\nu} .
$$

Note that $\sup _{u \geq 0} \sqrt{u} J_{\nu}(u)<\infty$.

Let

$$
\|f\|_{l^{1}\left(L^{\infty}\right)}=\sum_{\alpha \in \mathbb{Z}^{d}} \sup _{x \in C_{\alpha}}|f(x)|,
$$

where $C_{\alpha}$ denotes the unit cube centered at $\alpha \in \mathbb{Z}^{d}$. We introduce an additional assumption on distribution density $p_{t}$.

Assumption 4.2 Let $p_{t}$ be such that $\sup _{t>0}\left\|1_{\{|x|>\delta\}} p_{t}\right\|_{l^{1}\left(L^{\infty}\right)}<\infty$.

Let $f$ be a real valued function on $\mathbb{R}^{d}$. When $r \mapsto f(r x)$ is non-increasing on $[0, \infty)$, we say that $f$ is radially non-increasing. In $d=1$ for a radially non-increasing $L^{1}$-function $f$ it can be seen by the definition of $l^{1}\left(L^{\infty}\right)$ that there exists a constant $C_{\delta}=C_{\delta}(f)$ such that

$$
\left\|1_{\{|x|>\delta\}} f\right\|_{l^{1}\left(L^{\infty}\right)} \leq C_{\delta}\|f\|_{L^{1}} .
$$

In the general case $d \geq 2$ it can be also seen that (4.6) holds for all radially nonincreasing $f$, see [CMS90, p. 131, Corollary]. In particular, Assumption 4.2 is satisfied whenever $p_{t}$ is radially non-increasing, since $\left\|p_{t}\right\|_{L^{1}}=1$.

Example 4.3 ( $\alpha$-stable subordinator) In the case of $\Psi(u)=u^{\alpha}, 0 \leq \alpha \leq 1$, it is clear that Assumption 4.1 is satisfied. It is also known that the distribution density $p_{t}^{\alpha}$ of $B_{T_{t}^{\Psi}}$ is radially non-increasing. This is proven by a unimodality argument of spherically symmetric distribution functions; see [Kan77, Theorem 4.1], Wol78, Theorem 2], CMS90, p.132], Yam78, Theorem 1], and [Sat99] for details on unimodality. Then Assumption 4.2 is again satisfied. 
Example 4.4 Let $\Psi(u)=\sqrt{2 u+m^{2}}-m, m \geq 0$. It is clear that Assumption 4.1 is satisfied. The distribution function $p_{t}$ of $B_{T_{t}^{\Psi}}$ is expressed as

$$
p_{t}(x)=(2 \pi)^{-d} \frac{1}{\sqrt{|x|^{2}+t^{2}}} \int_{\mathbb{R}^{d}} e^{m t} e^{-\sqrt{\left(|x|^{2}+t^{2}\right)\left(p^{2}+m^{2}\right)}} d p
$$

see [HS78, (2.7)]. Then $p_{t}$ is indeed radially non-increasing.

The next proposition allows an extension of $\Psi\left(\mathrm{p}^{2} / 2\right)$ to Kato class.

Proposition 4.5 Let $V \geq 0$. Under Assumptions 4.1 and 4.2 the following three properties are equivalent:

(1) $\lim _{t \downarrow 0} \sup _{x \in \mathbb{R}^{d}} \int_{0}^{t} \mathbb{E}_{P \times \nu}^{x, 0}\left[V\left(X_{s}\right)\right] d s=0$,

(2) $\lim _{\lambda \rightarrow \infty} \sup _{x \in \mathbb{R}^{d}}\left(\left(\Psi\left(\mathrm{p}^{2} / 2\right)+\lambda\right)^{-1} V\right)(x)=0$,

(3) $\lim _{\delta \downarrow 0} \sup _{x \in \mathbb{R}^{d}} \int_{|x-y|<\delta} \Pi_{1}(x-y) V(y) d y=0$.

Proof. Similar to Theorem III.1 in CMS90.

qed

Definition 4.6 ( $\Psi$-Kato class) Take Assumptions 4.1 and 4.2 . Write $V=V_{+}-V_{-}$ in terms of its positive and negative parts. The $\Psi$-Kato class is defined as the set of potentials $V$ for which $V_{-}$and $1_{C} V_{+}$with every compact subset $C \subset \mathbb{R}^{d}$ satisfy any of the three equivalent conditions in Proposition 4.5. Here $1_{C}$ denotes the indicator function on $C$.

By (3) of Proposition 4.5 we can derive explicit conditions defining $\Psi$-Kato class using the relation of the Lévy measure of the subordinator with the associated Bernstein function.

Example 4.7 In the case $d=3$, since $J_{1 / 2}(x)=(2 / \pi)^{1 / 2} x^{-1 / 2} \sin x$, we have

$$
\Pi_{\lambda}(x)=\frac{1}{2 \pi^{2}|x|} \int_{0}^{\infty} \frac{r \sin r}{|x|^{2}\left(\lambda+\Psi\left(\frac{r^{2}}{2|x|^{2}}\right)\right)} d r
$$


Example 4.8 Let $T_{t}$ be an $\alpha$-stable process generated by $\left((1 / 2) \mathrm{p}^{2}\right)^{\alpha}, \alpha \in(0,1)$. Then a calculation gives that $V$ is in $\Psi$-Kato class in the sense of Definition 4.6 if and only if

$$
\Pi_{1}(x)= \begin{cases}c(d, 2 \alpha)|x|^{2 \alpha-d}, & 2 \alpha<d \\ -\frac{1}{\pi} \log |x|, & 2 \alpha=d \in\{1,2\} \\ c(1,2 \alpha)|x|^{2 \alpha-1}, & 2 \alpha>d=1,\end{cases}
$$

where $c(d, \beta):=\frac{\Gamma((d-\beta) / 2)}{2^{\beta} \pi^{d / 2}|\Gamma(\beta / 2)|}$.

Remark 4.9 For $\Psi$-Kato class potentials $V$ condition (2) of Proposition 4.5 implies that $V_{-}$is infinitesimally form bounded with respect to $\Psi\left(\mathrm{p}^{2} / 2\right)$. In this case $\Psi\left(\mathrm{p}^{2} / 2\right)+$ $V$ can be defined in form sense.

\section{2 $\Psi$-Kato class potential and hypercontractivity}

In this section we construct Schrödinger semigroups with $\Psi$-Kato class potentials and show their hypercontractivity property. References on the hypercontractivity for semigroups with usual Schrödinger operators with magnetic field include [Sim82, BHL00].

Lemma 4.10 Let $V \geq 0$ and $\Psi \in \mathscr{B}_{0}$. Suppose that $V$ satisfies (1) of Proposition 4.5 . Then for $t \geq 0$,

$$
\sup _{x \in \mathbb{R}^{d}} \mathbb{E}_{P \times \nu}^{x, 0}\left[e^{\int_{0}^{t} V\left(X_{s}\right) d s}\right]<\infty
$$

Proof. There exists $s>0$ such that $\sup _{x \in \mathbb{R}^{d}} \mathbb{E}_{P \times \nu}^{x, 0}\left[\int_{0}^{s} V\left(X_{s}\right) d s\right]=\epsilon<1$ by (1) of Proposition 4.5. Then by the Khas'minskii Lemma we conclude that

$$
\sup _{x \in \mathbb{R}^{d}} \mathbb{E}_{P \times \nu}^{x, 0}\left[e^{\int_{0}^{s} V\left(X_{s}\right) d s}\right] \leq(1-\epsilon)^{-1}
$$

Consider the image measure $\rho$ of $\left(X_{t}\right)_{t \geq 0}$ on the space $D\left([0, \infty) ; \mathbb{R}^{d}\right)$ of cádlág paths. Then $\mathbb{E}_{\rho}^{x}\left[e^{\int_{0}^{s} V\left(X_{s}\right) d s}\right]=\mathbb{E}_{P \times \nu}^{x, 0}\left[e^{\int_{0}^{s} V\left(X_{s}\right) d s}\right]$ and clearly $\left(X_{t}\right)_{t \geq 0}$ is a Markov process with respect to $\rho$. Furthermore,

$$
\begin{aligned}
\mathbb{E}_{\rho}^{x}\left[e^{\int_{0}^{2 s} V\left(X_{s}\right) d s}\right] & =\mathbb{E}_{\rho}^{x}\left[e^{\int_{0}^{s} V\left(X_{s}\right) d s} e^{\int_{s}^{2 s} V\left(X_{s}\right) d s}\right] \\
& =\mathbb{E}_{\rho}^{x}\left[e^{\int_{0}^{s} V\left(X_{s}\right) d s} \mathbb{E}_{\rho}^{X_{s}}\left[e^{\int_{0}^{s} V\left(X_{s}\right) d s}\right]\right] \\
& \leq\left(\sup _{y \in \mathbb{R}^{d}} \mathbb{E}_{\rho}^{y}\left[e^{\int_{0}^{s} V\left(X_{s}\right) d s}\right]\right) \mathbb{E}_{\rho}^{x}\left[e^{\int_{0}^{s} V\left(X_{s}\right) d s}\right] \\
& \leq(1-\epsilon)^{-2}
\end{aligned}
$$


Repeating this procedure we obtain (4.7) for all $t \geq 0$.

qed

The next result says that we can define a Feynman-Kac semigroup for $\Psi$-Kato class potentials.

Theorem 4.11 Let $\Psi \in \mathscr{B}_{0}, V$ belong to $\Psi$-Kato class and let Assumption (A2) hold. Consider

$$
\mathrm{U}_{t} f(x)=\mathbb{E}_{P \times \nu}^{x, 0}\left[e^{-i \int_{0}^{T_{t}^{\Psi}} a\left(B_{s}\right) o d B_{s}} e^{-\int_{0}^{t} V\left(B_{T_{s}^{\Psi}}\right) d s} f\left(B_{T_{t}^{\Psi}}\right)\right] .
$$

Then $\mathrm{U}_{t}$ is a strongly continuous symmetric semigroup. In particular, there exists a self-adjoint operator $K^{\Psi}$ bounded from below such that $\mathrm{U}_{t}=e^{-t K^{\Psi}}$.

Proof. Let $V=V_{+}-V_{-}$. Hence by Lemma 4.10 we have

$$
\begin{aligned}
\left\|\mathrm{U}_{t} f\right\|^{2} & \leq \int_{\mathbb{R}^{d}} d x \mathbb{E}_{P \times \nu}^{x, 0}\left[e^{-2 \int_{0}^{t} V_{+}\left(X_{s}\right) d s}\left|f\left(X_{t}\right)\right|^{2}\right] \mathbb{E}_{P \times \nu}^{x, 0}\left[e^{2 \int_{0}^{t} V_{-}\left(X_{s}\right) d s}\right] \\
& \left.\leq C_{t} \int_{\mathbb{R}^{d}} d x \mathbb{E}_{P \times \nu}^{x, 0}\left|f\left(X_{t}\right)\right|^{2}\right] \\
& =C_{t}\left\|e^{-t \Psi\left(\mathrm{p}^{2} / 2\right)} f\right\|^{2} \leq C_{t}\|f\|^{2},
\end{aligned}
$$

where $C_{t}=\sup _{x \in \mathbb{R}^{d}} \mathbb{E}_{P \times \nu}^{x, 0}\left[e^{2 \int_{0}^{t} V_{-}\left(X_{s}\right) d s}\right]$. Thus $\mathrm{U}_{t}$ is a bounded operator from $L^{2}\left(\mathbb{R}^{d}\right)$ to $L^{2}\left(\mathbb{R}^{d}\right)$. In the same manner as in Step 2 of the proof of Theorem 3.8 we conclude that the semigroup property $\mathrm{U}_{t} \mathrm{U}_{s}=\mathrm{U}_{t+s}$ holds for $t, s \geq 0$. We check strong continuity of $\mathrm{U}_{t}$ in $t$; it suffices to show weak continuity. Let $f, g \in C_{0}^{\infty}\left(\mathbb{R}^{d}\right)$ and simply we write $T_{t}$ for $T_{t}^{\Psi}$. Then we have

$$
\left(f, \mathrm{U}_{t} g\right)=\int_{\mathbb{R}^{d}} d x \mathbb{E}_{P \times \nu}^{x, 0}\left[\overline{f\left(B_{0}\right)} g\left(B_{T_{t}}\right) e^{-i \int_{0}^{T_{t}} a\left(B_{s}\right) o d B_{s}} e^{-\int_{0}^{t} V\left(B_{T_{s}}\right) d s}\right] .
$$

Since $T_{t}(\tau) \rightarrow 0$ as $t \rightarrow 0$ for each $\tau \in \Omega_{\nu}$, the dominated convergence theorem gives $\left(f, \mathrm{U}_{t} g\right) \rightarrow(f, g)$.

Finally we check the symmetry property $\mathrm{U}_{t}^{*}=\mathrm{U}_{t}$. By a limiting argument it is enough to show this for $a \in\left(C_{\mathrm{b}}^{2}\left(\mathbb{R}^{d}\right)\right)^{d}$. Let $\widetilde{B}_{s}=\widetilde{B}_{s}(\omega, \tau)=B_{T_{t}(\tau)-s}(\omega)-B_{T_{t}(\tau)}(\omega)$. Then for each $\tau \in \Omega_{\nu}, \widetilde{B}_{s} \stackrel{\mathrm{d}}{=} B_{s}$ with respect to $d P^{x}$. (Here $Z \stackrel{\mathrm{d}}{=} Y$ denotes that $Z$ and $Y$ are identically distributed.) Thus there exists a sequnece $\{n\} \subset \mathbb{N}$ such that

$$
\begin{aligned}
\left(f, \mathrm{U}_{t} g\right) & =\mathbb{E}_{P \times \nu}^{0,0}\left[\int_{\mathbb{R}^{d}} d x \overline{f(x)} e^{-i \int_{0}^{T_{t}} a\left(x+\widetilde{B}_{s}\right) \circ d \widetilde{B}_{s}} e^{-\int_{0}^{t} V\left(x+\widetilde{B}_{T_{s}}\right)} g\left(x+\widetilde{B}_{T_{t}}\right)\right] \\
& =\lim _{n \rightarrow \infty} \mathbb{E}_{P \times \nu}^{0,0}\left[\int_{\mathbb{R}^{d}} d x \overline{f(x)} e^{-i \sum_{j=1}^{n} I_{j}} e^{-\int_{0}^{t} V\left(x+\widetilde{B}_{T_{s}}\right)} g\left(x+\widetilde{B}_{T_{t}}\right)\right],
\end{aligned}
$$


where $I_{j}=\frac{1}{2}\left(a\left(x+\widetilde{B}_{T_{t} j / n}\right)+a\left(x+\widetilde{B}_{T_{t}(j-1) / n}\right)\right)\left(B_{T_{t} j / n}-B_{T_{t}(j-1) / n}\right)$. Changing the variable $x$ to $y=x+\widetilde{B}_{T_{t}}$, we have

$$
\left(f, \mathrm{U}_{t} g\right)=\lim _{n \rightarrow \infty} \mathbb{E}_{P \times \nu}^{0,0}\left[\int_{\mathbb{R}^{d}} d y \overline{f\left(y-\widetilde{B}_{T_{t}}\right)} e^{-i \sum_{j=1}^{n} \widetilde{I}_{j}} e^{-\int_{0}^{t} V\left(y-\widetilde{B}_{T_{t}}+\widetilde{B}_{T_{s}}\right)} g(y)\right],
$$

where

$$
\widetilde{I}_{j}=\frac{1}{2}\left(a\left(y-\widetilde{B}_{T_{t}}+\widetilde{B}_{T_{t} j / n}\right)+a\left(y-\widetilde{B}_{T_{t}}+\widetilde{B}_{T_{t}(j-1) / n}\right)\right)\left(\widetilde{B}_{T_{t} j / n}-\widetilde{B}_{T_{t}(j-1) / n}\right) .
$$

Since $\widetilde{B}_{T_{s}}-\widetilde{B}_{T_{t}} \stackrel{\mathrm{d}}{=} B_{T_{t}-T_{s}}$, we can compute $\lim _{n \rightarrow \infty} \sum_{j=1}^{n} \widetilde{I}_{j}$ in $L^{2}\left(\Omega_{P}, d P^{0}\right)$ as

$$
\begin{aligned}
& \lim _{n \rightarrow \infty} \sum_{j=1}^{n} \widetilde{I}_{j} \\
& =\lim _{n \rightarrow \infty} \sum_{j=1}^{n} \frac{1}{2}\left(a\left(y+B_{T_{t}-T_{t} j / n}\right)+a\left(y+B_{T_{t}-T_{t}(j-1) / n}\right)\right)\left(B_{T_{t}-T_{t} j / n}-B_{T_{t}-T_{t}(j-1) / n}\right) \\
& =-\lim _{n \rightarrow \infty} \sum_{j=1}^{n} \frac{1}{2}\left(a\left(y+B_{T_{t} j / n}\right)+a\left(y+B_{T_{t}(j-1) / n}\right)\right)\left(B_{T_{t} j / n}-B_{T_{t}(j-1) / n}\right) \\
& =-\int_{0}^{T_{t}} a\left(B_{s}\right) \circ d B_{s} .
\end{aligned}
$$

Then we have

$$
\left(f, \mathrm{U}_{t} g\right)=\int_{\mathbb{R}^{d}} d x \mathbb{E}_{P \times \nu}^{x, 0}\left[\overline{f\left(B_{T_{t}}\right)} e^{+i \int_{0}^{T_{t}} a\left(B_{s}\right) \circ d B_{s}} e^{-\int_{0}^{t} V\left(B_{T_{t}-T_{s}}\right) d s} g(x)\right] .
$$

Moreover, as $T_{t}-T_{s} \stackrel{\mathrm{d}}{=} T_{t-s}$ for $0 \leq s \leq t$, we obtain

$$
\begin{aligned}
\left(f, \mathrm{U}_{t} g\right) & =\int_{\mathbb{R}^{d}} d x \mathbb{E}_{P \times \nu}^{x, 0}\left[\overline{f\left(B_{T_{t}}\right)} e^{+i \int_{0}^{T_{t}} a\left(B_{s}\right) \circ d B_{s}} e^{-\int_{0}^{t} V\left(B_{T_{t-s}}\right) d s} g(x)\right] \\
& =\int_{\mathbb{R}^{d}} d x \overline{\mathbb{E}_{P \times \nu}^{x, 0}\left[f\left(B_{T_{t}}\right) e^{-i \int_{0}^{T_{t}} a\left(B_{s}\right) \circ d B_{s}} e^{-\int_{0}^{t} V\left(B_{T_{s}}\right) d s}\right]} g(x) \\
& =\left(\mathrm{U}_{t} f, g\right) .
\end{aligned}
$$

The existence of a self-adjoint operator $K^{\Psi}$ bounded from below such that $\mathrm{U}_{t}=e^{-t K^{\Psi}}$ is a consequence of the Hille-Yoshida theorem. This completes the proof. qed

Definition 4.12 ( $\Psi$-Kato class Schrödinger operator) Let $V$ be in $\Psi$-Kato class and take Assumption (A2). We call $K^{\Psi}$ given in Theorem4.11 generalized Schrödinger operator for $\Psi$-Kato class potentials. We refer to the one-parameter operator semigroup $e^{-t K^{\Psi}}, t \geq 0$, as the $\Psi$-Kato class generalized Schrödinger semigroup. 
Put $K_{0}^{\Psi}$ for the operator defined by $K^{\Psi}$ with a replaced by 0 .

Theorem 4.13 (Hypercontractivity) Let $V$ be a $\Psi$-Kato class potential and assume (A2) to hold. Then $e^{-t K^{\Psi}}$ is a bounded operator from $L^{p}\left(\mathbb{R}^{d}\right)$ to $L^{q}\left(\mathbb{R}^{d}\right)$, for all $1 \leq$ $p \leq q \leq \infty$. Moreover, $\left\|e^{-t K^{\Psi}}\right\|_{p, q} \leq\left\|e^{-t K_{0}^{\Psi}}\right\|_{p, q}$ holds for all $t \geq 0$.

Proof. By the Riesz-Thorin theorem it suffices to show that $e^{-t K^{\Psi}}$ is bounded as an operator of $(1) L^{\infty}\left(\mathbb{R}^{d}\right) \rightarrow L^{\infty}\left(\mathbb{R}^{d}\right)$, (2) $L^{1}\left(\mathbb{R}^{d}\right) \rightarrow L^{1}\left(\mathbb{R}^{d}\right)$ and $(3) L^{1}\left(\mathbb{R}^{d}\right) \rightarrow L^{\infty}\left(\mathbb{R}^{d}\right)$. Since

$$
\left|e^{-t K^{\Psi}} f(x)\right| \leq e^{-t K_{0}^{\Psi}}|f|(x),
$$

we will prove (1)-(3) for $e^{-t K_{0}^{\Psi}}$. For simplicity we denote $\mathbb{E}_{P \times \nu}^{x, 0}=\mathbb{E}^{x}$ and $\mathrm{P}_{t}=e^{-t K_{0}^{\Psi}}$, i.e., we have

$$
\mathrm{P}_{t} f(x)=\mathbb{E}^{x}\left[e^{-\int_{0}^{t} V\left(X_{s}\right) d s} f\left(X_{t}\right)\right]
$$

To consider $(1)$, let $f \in L^{\infty}\left(\mathbb{R}^{d}\right)$. We have by Lemma 4.10,

$$
\left\|\mathrm{P}_{t} f\right\|_{\infty} \leq \sup _{x \in \mathbb{R}^{d}}\left(\mathbb{E}^{x}\left[e^{-\int_{0}^{t} V\left(X_{s}\right) d s}\right]\right)\|f\|_{\infty}
$$

Thus (1) follows.

To derive (2), let $0 \leq f \in L^{1}\left(\mathbb{R}^{d}\right)$ and $g \equiv 1 \in L^{\infty}\left(\mathbb{R}^{d}\right)$. Then $\mathrm{P}_{t} g \in L^{\infty}\left(\mathbb{R}^{d}\right)$ by $(1)$ above. In the same way as in the proof of the symmetry of $U_{t}$ in Theorem 4.11 it can be shown that

$$
\int_{\mathbb{R}^{d}} d x f(x) \cdot \mathrm{P}_{t} g(x)=\int_{\mathbb{R}^{d}} d x \mathrm{P}_{t} f(x) \cdot g(x)=\int_{\mathbb{R}^{d}} d x \mathrm{P}_{t} f(x) .
$$

Since $\mathrm{P}_{t} f(x) \geq 0$, we have $\left\|\mathrm{P}_{t} f\right\|_{1} \leq\|f\|_{1}\left\|\mathrm{P}_{t} 1\right\|_{\infty}$. Taking any $f \in L^{1}\left(\mathbb{R}^{d}\right)$ and splitting it off as $f=\Re f_{+}-\Re f_{-}+i\left(\Im f_{+}-\Im f_{-}\right)$, we get $\left\|\mathrm{P}_{t}\right\|_{1} \leq 4\|f\|_{1}\left\|\mathrm{P}_{t} 1\right\|_{\infty}$. This gives (2).

Combining (1) and (2) with the Riesz-Thorin theorem we deduce that $\mathrm{P}_{t}$ is a bounded operator from $L^{p}\left(\mathbb{R}^{d}\right)$ to $L^{p}\left(\mathbb{R}^{d}\right)$, for all $1 \leq p \leq \infty$. Moreover, the Markov property of $\left(X_{t}\right)_{t \geq 0}$ implies that $\mathrm{P}_{t}$ is a semigroup on $L^{p}\left(\mathbb{R}^{d}\right)$, for $1 \leq p \leq \infty$.

Finally we consider (3) with the diagram

$$
L^{1}\left(\mathbb{R}^{d}\right) \stackrel{\mathrm{P}_{t}}{\longrightarrow} L^{2}\left(\mathbb{R}^{d}\right) \stackrel{\mathrm{P}_{t}}{\longrightarrow} L^{\infty}\left(\mathbb{R}^{d}\right) .
$$

Let $f \in L^{2}\left(\mathbb{R}^{d}\right)$. Then

$$
\left|\mathrm{P}_{t} f(x)\right|^{2} \leq \mathbb{E}^{x}\left[e^{-2 \int_{0}^{t} V\left(X_{s}\right) d s}\right] \mathbb{E}^{x}\left[\left|f\left(X_{t}\right)\right|^{2}\right] \leq C_{t} \int_{\mathbb{R}^{d}}|f(x+y)|^{2} p_{t}(y) d y
$$


by Lemma 4.10, where $C_{t}=\sup _{x \in \mathbb{R}^{d}} \mathbb{E}^{x}\left[e^{-\int_{0}^{t} V\left(X_{s}\right) d s}\right]$. Since

$$
\left|p_{t}(y)\right| \leq \int_{0}^{\infty} e^{-t \Psi\left(u^{2} / 2\right)} d u<\infty
$$

by Assumption 4.1, with $p_{t}$ in (4.4), it follows that

$$
\left\|\mathrm{P}_{t} f\right\|_{\infty} \leq\left(C_{t}\left\|p_{t}\right\|_{\infty}\right)^{1 / 2}\|f\|_{2}
$$

Thus $\mathrm{P}_{t}$ is a bounded operator from $L^{2}\left(\mathbb{R}^{d}\right)$ to $L^{\infty}\left(\mathbb{R}^{d}\right)$. Next, let $f \in L^{1}\left(\mathbb{R}^{d}\right)$ and $g \in L^{2}\left(\mathbb{R}^{d}\right)$. We have $\int_{\mathbb{R}^{d}} d x \mathrm{P}_{t} f(x) \cdot g(x)=\int_{\mathbb{R}^{d}} d x f(x) \cdot \mathrm{P}_{t} g(x)$. Then by (4.10) we obtain

$$
\left|\int_{\mathbb{R}^{d}} d x \mathrm{P}_{t} f(x) \cdot g(x)\right| \leq\left\|\mathrm{P}_{t} g\right\|_{\infty}\|f\|_{1} \leq\left(C_{t}\left\|p_{t}\right\|_{\infty}\right)^{1 / 2}\|g\|_{2}\|f\|_{1} .
$$

Since $g \in L^{2}\left(\mathbb{R}^{d}\right)$ is arbitrary, $\mathrm{P}_{t} f \in L^{2}\left(\mathbb{R}^{d}\right)$ and

$$
\left\|\mathrm{P}_{t} f\right\|_{2} \leq\left(C_{t}\left\|p_{t}\right\|_{\infty}\right)^{1 / 2}\|f\|_{1}
$$

follows, hence $\mathrm{P}_{t}$ is a bounded operator from $L^{1}\left(\mathbb{R}^{d}\right)$ to $L^{2}\left(\mathbb{R}^{d}\right)$. Thus (4.9) holds.

By the semigroup property and (4.9) we have for $f \in L^{1}\left(\mathbb{R}^{d}\right)$,

$$
\left\|\mathrm{P}_{t} f\right\|_{\infty}=\left\|\mathrm{P}_{t / 2} \mathrm{P}_{t / 2} f\right\|_{\infty} \leq\left(C_{t / 2}\left\|p_{t / 2}\right\|_{\infty}\right)^{1 / 2}\left\|\mathrm{P}_{t / 2} f\right\|_{2} \leq C_{t / 2}\left\|p_{t / 2}\right\|_{\infty}\|f\|_{1}
$$

The fact $\left\|e^{-t K^{\Psi}}\right\|_{p, q} \leq\left\|e^{-t K_{0}^{\Psi}}\right\|_{p, q}$ follows from (4.8). This completes the proof of the theorem.

qed

\section{The case of operators with spin}

\subsection{Schrödinger operator with spin $1 / 2$}

Besides operators describing interactions with magnetic fields we now consider operators also including a spin variable. The Schrödinger operator with spin $1 / 2$ is formally given by

$$
h_{1 / 2}=\frac{1}{2}(\sigma \cdot(\mathrm{p}-a))^{2}
$$

on $L^{2}\left(\mathbb{R}^{3} ; \mathbb{C}^{2}\right)$, where $\sigma=\left(\sigma_{1}, \sigma_{2}, \sigma_{3}\right)$ are the Pauli matrices

$$
\sigma_{1}:=\left[\begin{array}{ll}
0 & 1 \\
1 & 0
\end{array}\right], \quad \sigma_{2}:=\left[\begin{array}{cc}
0 & -i \\
i & 0
\end{array}\right], \quad \sigma_{3}:=\left[\begin{array}{cc}
1 & 0 \\
0 & -1
\end{array}\right]
$$


satisfying $\left\{\sigma_{\mu}, \sigma_{\nu}\right\}=2 \delta_{\mu \nu} 1$ and $\sigma_{\mu} \sigma_{\nu}=i \sum_{\lambda=1}^{3} \epsilon^{\lambda \mu \nu} \sigma_{\lambda}$, where $\epsilon^{\lambda \mu \nu}$ is the anti-symmetric Levi-Cività tensor with $\epsilon^{123}=1$. We use the identification $L^{2}\left(\mathbb{R}^{3} ; \mathbb{C}^{2}\right) \cong \mathbb{C}^{2} \otimes L^{2}\left(\mathbb{R}^{3}\right)$. A rigorous definition of $h_{1 / 2}$ can be given through a quadratic form in the same fashion as in the spinless case. Define the quadratic form

$$
q_{1 / 2}(f, g)=\sum_{\mu=1}^{3}\left(\sigma_{\mu} \mathrm{D}_{\mu} f, \sigma_{\mu} \mathrm{D}_{\mu} g\right)
$$

with domain

$$
Q\left(q_{1 / 2}\right)=\left\{f \in L^{2}\left(\mathbb{R}^{3} ; \mathbb{C}^{2}\right) \mid \sigma_{\mu} \mathrm{D}_{\mu} f \in L^{2}\left(\mathbb{R}^{3} ; \mathbb{C}^{2}\right), \mu=1,2,3\right\}
$$

Assume (A1); then $q_{1 / 2}$ is nonnegative and closed. By this property there exists a unique self-adjoint operator $h_{1 / 2}$ satisfying

$$
\left(h_{1 / 2} f, g\right)=q_{1 / 2}(f, g), \quad f \in D\left(h_{1 / 2}\right), g \in Q\left(q_{1 / 2}\right),
$$

where

$$
D\left(h_{1 / 2}\right)=\left\{f \in Q\left(q_{1 / 2}\right) \mid q_{1 / 2}(f, \cdot) \in L^{2}\left(\mathbb{R}^{3} ; \mathbb{C}^{2}\right)^{\prime}\right\}
$$

Theorem 5.1 The following holds on the cores of $h_{1 / 2}$ :

(1) Let Assumption (A1) hold with $d=3$. Then $\mathbb{C}^{2} \otimes C_{0}^{\infty}\left(\mathbb{R}^{3}\right)$ is a form core of $h_{1 / 2}$.

(2) Let Assumption (A4) hold. Then $\mathbb{C}^{2} \otimes C_{0}^{\infty}\left(\mathbb{R}^{3}\right)$ is an operator core of $h_{1 / 2}$.

Let $\Psi \in \mathscr{B}_{0}$. Then furthermore the following holds on the cores of $\Psi\left(h_{1 / 2}\right)$ :

(3) Take Assumption (A1) with $d=3$. Then $\mathbb{C}^{2} \otimes C_{0}^{\infty}\left(\mathbb{R}^{3}\right)$ is a form core of $\Psi\left(h_{1 / 2}\right)$.

(4) Take Assumption (A4). Then $\mathbb{C}^{2} \otimes C_{0}^{\infty}\left(\mathbb{R}^{3}\right)$ is an operator core of $\Psi\left(h_{1 / 2}\right)$.

Proof. The proofs of (1) and (2) are similar to that of Proposition 3.1, while those of (3) and (4) can be proven in the same way as in Theorem 3.3 qed

Note that under Assumption (A4)

$$
h_{1 / 2} f=\frac{1}{2} \mathrm{p}^{2} f-a \cdot \mathrm{p} f+\left(-\frac{1}{2} a \cdot a-(\mathrm{p} \cdot a)-\frac{1}{2} \sigma \cdot(\nabla \times a)\right) f
$$


holds for $f \in \mathbb{C}^{2} \otimes C_{0}^{\infty}\left(\mathbb{R}^{3}\right)$. In order to construct a functional integral representation for $e^{-t h_{1 / 2}}$ we make a unitary transform of the operator $h_{1 / 2}$ on $L^{2}\left(\mathbb{R}^{3} ; \mathbb{C}^{2}\right)$ to an operator on the space $L^{2}\left(\mathbb{R}^{3} \times \mathbb{Z}_{2}\right)$. This is a space of $L^{2}$-functions of $x \in \mathbb{R}^{3}$ and an additional two-valued spin variable $\theta \in \mathbb{Z}_{2}$, where

$$
\mathbb{Z}_{2}=\{-1,1\}=\left\{\theta_{1}, \theta_{2}\right\}
$$

Also, we define on $L^{2}\left(\mathbb{R}^{3} \times \mathbb{Z}_{2}\right)$ the operator

$$
\left(h_{\mathbb{Z}_{2}} f\right)(x, \theta):=(h f)(x, \theta)-\frac{1}{2} \theta b_{3}(x) f(x, \theta)-\frac{1}{2}\left(b_{1}(x)-i \theta b_{2}(x)\right) f(x,-\theta),
$$

where $x \in \mathbb{R}^{3}, \theta \in \mathbb{Z}_{2}$ and

$$
\left(b_{1}, b_{2}, b_{3}\right)=\nabla \times a .
$$

The closure of $h_{\mathbb{Z}_{2}}\left\lceil\mathbb{C}^{2} \otimes C_{0}^{\infty}\left(\mathbb{R}^{3}\right)\right.$ will be denoted by the same symbol $h_{\mathbb{Z}_{2}}$. Also, we use the identification $L^{2}\left(\mathbb{R}^{3} \times \mathbb{Z}_{2}\right) \cong \ell^{2}\left(\mathbb{Z}_{2}\right) \otimes L^{2}\left(\mathbb{R}^{3}\right)$. The operators $h_{\mathbb{Z}_{2}}$ and $h_{1 / 2}$ are unitary equivalent, as seen below. Define the unitary operator

$$
\mathrm{F}: L^{2}\left(\mathbb{R}^{3} \times \mathbb{Z}_{2}\right) \rightarrow \mathbb{C}^{2} \otimes L^{2}\left(\mathbb{R}^{3}\right) \cong L^{2}\left(\mathbb{R}^{3}\right) \oplus L^{2}\left(\mathbb{R}^{3}\right)
$$

by

$$
\mathrm{F} f=\left[\begin{array}{l}
f(\cdot,+1) \\
f(\cdot,-1)
\end{array}\right], \quad f \in L^{2}\left(\mathbb{R}^{3} \times \mathbb{Z}_{2}\right)
$$

Proposition 5.2 Under Assumption $(A 4), h_{\mathbb{Z}_{2}}$ is self-adjoint on $\ell^{2}\left(\mathbb{Z}_{2}\right) \otimes D(h)$ and essentially self-adjoint on $\ell^{2}\left(\mathbb{Z}_{2}\right) \otimes C_{0}^{\infty}\left(\mathbb{R}^{3}\right)$. Moreover, it follows that

$$
\mathrm{F} h_{1 / 2} \mathrm{~F}^{-1}=h_{\mathbb{Z}_{2}}
$$

Proof. It can be directly seen that $\mathrm{F} h_{1 / 2} \mathrm{~F}^{-1}=h_{\mathbb{Z}_{2}}$ holds on $\ell^{2}\left(\mathbb{Z}_{2}\right) \otimes C_{0}^{\infty}\left(\mathbb{R}^{3}\right)$ and $\mathrm{F}$ maps $\ell^{2}\left(\mathbb{Z}_{2}\right) \otimes C_{0}^{\infty}\left(\mathbb{R}^{3}\right)$ onto $\mathbb{C}^{2} \otimes C_{0}^{\infty}\left(\mathbb{R}^{3}\right)$. Moreover, $\mathbb{C}^{2} \otimes C_{0}^{\infty}\left(\mathbb{R}^{3}\right)$ is a core of $h_{1 / 2}$ by Theorem 5.1, which yields the proposition.

qed

\subsection{Generalized Schrödinger operator with spin $\mathbb{Z}_{p}, p \geq 2$}

Next we generalize $h_{\mathbb{Z}_{2}}$ on $L^{2}\left(\mathbb{R}^{3} \times \mathbb{Z}_{2}\right)$ to consider an operator on $L^{2}\left(\mathbb{R}^{d} \times \mathbb{Z}_{p}\right)$ for $d \geq 1$ and $p \geq 2$. Define $\mathbb{Z}_{p}$ as the cyclic group of the $p$ th roots of unity by

$$
\mathbb{Z}_{p}=\left\{\theta_{1}^{(p)}, \ldots, \theta_{p}^{(p)}\right\}
$$


where

$$
\theta_{\alpha}^{(p)}=\exp \left(2 \pi i \frac{\alpha}{p}\right), \quad \alpha \in \mathbb{N}
$$

In what follows we fix $p \geq 2$ and abbreviate $\theta_{\beta}^{(p)}$ simply to $\theta_{\beta}$ for notational convenience. Consider the finite dimensional vector space $\ell^{2}\left(\mathbb{Z}_{p}\right)=\left\{f: \mathbb{Z}_{p} \rightarrow \mathbb{C}\right\}$ equipped with the scalar product $(f, g)_{\ell^{2}\left(\mathbb{Z}_{p}\right)}=\sum_{\beta=1}^{p} \overline{f\left(\theta_{\beta}\right)} g\left(\theta_{\beta}\right)$.

Now we consider the Schrödinger operator with spin $\mathbb{Z}_{p}$. We define a spin operator with its diagonal part $U$ and off-diagonal part $U_{\beta}, \beta=1, \ldots, p-1$, separately.

Definition 5.3 (Generalized spin operator) We define two functions below:

(1) (Diagonal part) Let $U: \mathbb{R}^{d} \times \mathbb{Z}_{p} \rightarrow \mathbb{R}$ be such that $\max _{\theta \in \mathbb{Z}_{p}}|U(x, \theta)|$ is a multiplication operator, relatively bounded with respect to $\frac{1}{2} \mathrm{p}^{2}$.

(2) (Off-diagonal part) Let $W_{\beta}: \mathbb{R}^{d} \times \mathbb{Z}_{p} \rightarrow \mathbb{C}, 1 \leq \beta \leq p-1$, be such that $\max _{\theta \in \mathbb{Z}_{p}}\left|W_{\beta}(x, \theta)\right|$ is a multiplication operator, relatively bounded with respect to $\frac{1}{2} \mathrm{p}^{2}$. Moreover, let $U_{\beta}: \mathbb{R}^{d} \times \mathbb{Z}_{p} \rightarrow \mathbb{C}$ be defined

$$
U_{\beta}\left(x, \theta_{\alpha}\right)=\frac{1}{2}\left(W_{\beta}\left(x, \theta_{\alpha+\beta}\right)+\overline{W_{p-\beta}\left(x, \theta_{\alpha}\right)}\right), \quad \alpha=1, \ldots, p, \beta=1, \ldots, p-1 .
$$

Furthermore, we call $M_{\mathbb{Z}_{p}}: L^{2}\left(\mathbb{R}^{d} \times \mathbb{Z}_{p}\right) \rightarrow L^{2}\left(\mathbb{R}^{d} \times \mathbb{Z}_{p}\right)$,

$$
M_{\mathbb{Z}_{p}}: f\left(x, \theta_{\alpha}\right) \mapsto U\left(x, \theta_{\alpha}\right) f\left(x, \theta_{\alpha}\right)+\sum_{\beta=1}^{p-1} U_{\beta}\left(x, \theta_{\alpha}\right) f\left(x, \theta_{\alpha+\beta}\right)
$$

generalized spin operator on $L^{2}\left(\mathbb{R}^{d} \times \mathbb{Z}_{p}\right)$.

Below we will use the notation

$$
u_{\beta}(x)= \begin{cases}\max _{\theta \in \mathbb{Z}_{p}}|U(x, \theta)| & \text { if } \beta=p, \\ \max _{\theta \in \mathbb{Z}_{p}}\left|U_{\beta}(x, \theta)\right| & \text { if } 1 \leq \beta \leq p-1 .\end{cases}
$$

Clearly, $u_{\beta}(x)$ is a multiplication operator relatively bounded with respect to $\frac{1}{2} \mathrm{p}^{2}$, i.e., there exist $c_{\beta}>0$ and $b_{\beta} \geq 0$ such that

$$
\left\|u_{\beta} f\right\| \leq c_{\beta}\left\|\frac{1}{2} \mathrm{p}^{2} f\right\|+b_{\beta}\|f\|, \quad \beta=1, \ldots, p,
$$

for all $f \in D\left((1 / 2) \mathrm{p}^{2}\right)$. These definitions of $U$ and $U_{\beta}$ cover, in particular, the $\mathbb{Z}_{2}$ case of the Schrödinger operator associated with spin $1 / 2$. 
Example $5.4($ Spin 1/2) Let $d=3$ and $p=2$. Define

$$
W_{1}(x, \theta)=-\frac{1}{2}\left(b_{1}(x)+i \theta b_{2}(x)\right), \quad \theta \in \mathbb{Z}_{2} .
$$

Then $\theta_{1}=-1, \theta_{2}=1$ and by (5.14) we see that

$$
U_{1}(x, \theta)=\frac{1}{2}\left(W_{1}\left(x, \theta \theta_{1}\right)+\overline{W_{1}(x, \theta)}\right), \quad \theta \in \mathbb{Z}_{2} .
$$

It is straightforward to see that $W_{1}\left(x, \theta \theta_{1}\right)=-\frac{1}{2}\left(b_{1}(x)-i \theta b_{2}(x)\right)=\overline{W_{1}(x, \theta)}$, hence the off-diagonal part is $U_{1}(x, \theta)=-\frac{1}{2}\left(b_{1}(x)-i \theta b_{2}(x)\right)$, while the diagonal part is given by $U(x, \theta)=-\frac{1}{2} \theta b_{3}(x)$, both of which coincide with the interaction in (5.7)

Example 5.5 Let $p \geq 2$, and $W_{\beta}(\theta)=W(\theta)=-\frac{1}{2}\left(b_{1}+i \theta b_{2}\right)$ for $1 \leq \beta \leq p-1$. Then

$$
U_{\beta}\left(\theta_{\alpha}\right)=\frac{1}{2}\left(W_{\beta}\left(\theta_{\alpha+\beta}\right)+\overline{W_{p-\beta}\left(\theta_{\alpha}\right)}\right)=-\frac{1}{2}\left(b_{1}+i \frac{\theta_{\alpha+\beta}-\theta_{p-\alpha}}{2} b_{2}\right) .
$$

This gives one possible generalization of the case of spin 1/2 of Example 5.4.

Definition 5.6 (Schrödinger operator with generalized spin) Let $h$ be the generalized Schrödinger operator defined in (3.3). Under Assumption (A1) we define the Schrödinger operator with generalized spin $M_{\mathbb{Z}_{p}}$ by

$$
h_{\mathbb{Z}_{p}}=1 \otimes h+M_{\mathbb{Z}_{p}}
$$

Above we made the identification $L^{2}\left(\mathbb{R}^{d} \times \mathbb{Z}_{p}\right) \cong \ell^{2}\left(\mathbb{Z}_{p}\right) \otimes L^{2}\left(\mathbb{R}^{d}\right)$. Formally, $h_{\mathbb{Z}_{p}}$ is written as

$$
\left(h_{\mathbb{Z}_{p}} f\right)\left(x, \theta_{\alpha}\right)=\left(\frac{1}{2}(\mathrm{p}-a(x))^{2}+U\left(x, \theta_{\alpha}\right)\right) f\left(x, \theta_{\alpha}\right)+\sum_{\beta=1}^{p-1} U_{\beta}\left(x, \theta_{\alpha}\right) f\left(x, \theta_{\alpha+\beta}\right) .
$$

Theorem 5.7 Take Assumption (A2) and let $U, U_{\beta}$ be given as in Definition 5.3 . Suppose $\sum_{\beta=1}^{p} c_{\beta}<1$, where $c_{\beta}$ is the constant in 5.17). Then $h_{\mathbb{Z}_{p}}$ is self-adjoint on $\ell^{2}\left(\mathbb{Z}_{p}\right) \otimes D(h)$ and bounded from below. Moreover, it is essentially self-adjoint on any core of $1 \otimes h$. In particular, $\ell^{2}\left(\mathbb{Z}_{p}\right) \otimes C_{0}^{\infty}\left(\mathbb{R}^{d}\right)$ is an operator core of $h_{\mathbb{Z}_{p}}$.

Proof. It can be seen that

$$
\sum_{\alpha=1}^{p} \overline{g\left(x, \theta_{\alpha}\right)}\left(\sum_{\beta=1}^{p-1} W_{\beta}\left(x, \theta_{\alpha+\beta}\right) f\left(x, \theta_{\alpha+\beta}\right)\right)=\sum_{\gamma=1}^{p}\left(\sum_{\beta=1}^{p-1} \overline{\overline{W_{p-\beta}\left(x, \theta_{\gamma}\right)} g\left(x, \theta_{\gamma+\beta}\right)}\right) f\left(x, \theta_{\gamma}\right)
$$


for each $x \in \mathbb{R}^{d}$. Then it follows that

$$
\left(g(x, \cdot), M_{\mathbb{Z}_{p}} f(x, \cdot)\right)_{\ell^{2}\left(\mathbb{Z}_{p}\right)}=\left(M_{\mathbb{Z}_{p}} g(x, \cdot), f(x, \cdot)\right)_{\ell^{2}\left(\mathbb{Z}_{p}\right)}
$$

and $M_{\mathbb{Z}_{p}}$ is symmetric. Its norm can be estimated as $\left\|M_{\mathbb{Z}_{p}} f\right\| \leq \sum_{\beta=1}^{p}\left\|\left(1 \otimes u_{\beta}\right) f\right\|$ by Definition 5.3. Then with $h_{0}=\frac{1}{2} \mathrm{p}^{2}$ and $E>0$, we have by (3.26) in the proof of Theorem 3.12, $\left\|u_{\beta}(h+E)^{-1} g\right\| \leq\left\|u_{\beta}\left(h_{0}+E\right)^{-1}|g|\right\|$ and hence

$$
\left\|M_{\mathbb{Z}_{p}} f\right\| \leq \sum_{\beta=1}^{p}\left\|u_{\beta}\left(h_{0}+E\right)^{-1}\right\|\|1 \otimes(h+E) f\| \leq \sum_{\beta=1}^{p} c_{\beta}\|(1 \otimes h) f\|+b\|f\|
$$

with a suitable constant $b$. Thus the claim follows by the Kato-Rellich theorem. qed

Definition 5.8 (Generalized Schrödinger operator with spin) Suppose that $U$ and $U_{\beta}$ are given as in Definition 5.3 and let Assumption (A2) hold. Moreover, assume that $\sum_{\beta=1}^{p} c_{\beta}<1$. Let $\Psi \in \mathscr{B}_{0}$ and put

$$
\overline{h_{\mathbb{Z}_{p}}}= \begin{cases}h_{\mathbb{Z}_{p}} & \text { if } \mathscr{E}_{\mathbb{Z}_{p}} \geq 0 \\ h_{\mathbb{Z}_{p}}-\mathscr{E}_{h_{\mathbb{Z}_{p}}} & \text { if } \mathscr{E}_{h_{\mathbb{Z}_{p}}}<0\end{cases}
$$

We call the operator

$$
H_{\mathbb{Z}_{p}}^{\Psi}=\Psi\left(\overline{h_{\mathbb{Z}_{p}}}\right)+V
$$

generalized Schrödinger operator with vector potential a and spin $\mathbb{Z}_{p}$.

Corollary 5.9 Let $U$ and $U_{\beta}$ be given as in Definition 5.3, assume (A2) and suppose that $\sum_{\beta=1}^{p} c_{\beta}<1$. If $\Psi \in \mathscr{B}_{0}$, then $\ell^{2}\left(\mathbb{Z}_{p}\right) \otimes C_{0}^{\infty}\left(\mathbb{R}^{d}\right)$ is an operator core of $\Psi\left(h_{\mathbb{Z}_{p}}\right)$.

Proof. Since $h_{\mathbb{Z}_{p}}$ is essentially self-adjoint on $\ell^{2}\left(\mathbb{Z}_{p}\right) \otimes C_{0}^{\infty}\left(\mathbb{R}^{d}\right)$, the corollary can be proven in the same way as Theorem 3.3 .

qed

\subsection{Functional integral representation}

In this subsection we give a functional integral representation of $e^{-t H_{\mathbb{Z}_{p}}^{\Psi}}$ by means of Brownian motion, a jump process and a subordinator.

Let $\left(N_{t}^{\beta}\right)_{t \geq 0}, \beta=1, \ldots, p-1$, be $p-1$ independent Poisson processes with unit intensity on a probability space $\left(\Omega_{N}, \mathscr{F}_{N}, \mu\right)$, i.e., $\mu\left(N_{t}^{\beta}=n\right)=e^{-t} t^{n} / n$ !. Define the random process $\left(N_{t}\right)_{t \geq 0}$ by

$$
N_{t}=\sum_{\beta=1}^{p-1} \beta N_{t}^{\beta}
$$


Let $\mathscr{F}_{t}^{N}=\sigma\left(N_{t}, t \leq s\right)$ be the natural filtration. Then since $N_{t}$ is a Lévy process, it is a Markov process with respect to $\mathscr{F}_{t}^{N}$. We write $\mathbb{E}_{\mu}\left[f\left(N_{t}+\alpha\right)\right]$ as $\mathbb{E}_{\mu}^{\alpha}\left[f\left(N_{t}\right)\right]$. Also, $\mathbb{E}_{\mu}^{\alpha}\left[N_{0}=\alpha\right]=1$. Define

$$
\int_{v}^{w+} g\left(N_{s-}\right) d N_{s}^{\beta}=\sum_{\substack{v \leq r \leq w \\ N_{r+}^{\beta} \neq N_{r-}^{\beta}}} g\left(N_{r-}\right) .
$$

It can be seen that

$$
\mathbb{E}_{\mu}\left[\int_{v}^{w+} g\left(N_{s-}\right) d N_{s}^{\beta}\right]=\mathbb{E}_{\mu}\left[\int_{v}^{w} g\left(N_{s}\right) d s\right]
$$

The next lemma is an extension of a result obtained in [ALS83, HL08].

Lemma 5.10 Let $U$ and $U_{\beta}$ be given in Definition 5.3 and assume $\sum_{\beta=1}^{p} c_{\beta}<1$. Suppose Assumption (A2). and

$$
\int_{0}^{t} d s \int_{\mathbb{R}^{d}} d y(2 \pi s)^{-d / 2} e^{-|x-y|^{2} /(2 s)}\left|\log u_{\beta}(y)\right|<\infty, \quad \beta=1, \ldots, p-1 .
$$

Then

$$
\left(f, e^{-t h_{\mathbb{Z}_{p}}} g\right)=e^{(p-1) t} \sum_{\alpha=1}^{p} \int_{\mathbb{R}^{d}} d x \mathbb{E}_{P \times \mu}^{x, \alpha}\left[\overline{f\left(B_{0}, \theta_{N_{0}}\right)} g\left(B_{t}, \theta_{N_{t}}\right) e^{\mathrm{S}}\right],
$$

where $\mathrm{S}=\mathrm{S}_{a}+\mathrm{S}_{\text {spin }}$ and

$$
\begin{aligned}
& \mathrm{S}_{a}=-i \int_{0}^{t} a\left(B_{s}\right) \circ d B_{s}, \\
& \mathrm{~S}_{\mathrm{spin}}=-\int_{0}^{t} U\left(B_{s}, \theta_{N_{s}}\right) d s+\sum_{\beta=1}^{p-1} \int_{0}^{t+} \log \left(-U_{\beta}\left(B_{s}, \theta_{N_{s-}}\right)\right) d N_{s}^{\beta} .
\end{aligned}
$$

Here we take $\log z$ with the principal branch for $z \in \mathbb{C}$.

Proof. First assume that the diagonal part $U\left(x, \theta_{\alpha}\right)$ and off-diagonal part $U_{\beta}\left(x, \theta_{\alpha}\right)$ are continuous in $x$ and $a \in\left(C_{0}^{\infty}\left(\mathbb{R}^{d}\right)\right)^{d}$. Since from (5.26) and (5.25) it follows that

$$
\mathbb{E}_{P \times \mu}^{x, \alpha}\left[\int_{0}^{t+}\left|\log \left(-U_{\beta}\left(B_{s}, \theta_{N_{s-}}\right)\right)\right| d N_{s}^{\beta}\right] \leq \int_{0}^{t} d s \int_{\mathbb{R}^{d}} \frac{e^{-|x-y|^{2} /(2 s)}}{(2 \pi s)^{d / 2}}\left|\log u_{\beta}(y)\right|<\infty,
$$

we note that

$$
\int_{0}^{t+}\left|\log \left(-U_{\beta}\left(B_{s}, \theta_{N_{s-}}\right)\right)\right| d N_{s}^{\beta}<\infty
$$


almost surely. By the estimate $\left|c \mathrm{~S}_{\text {spin }}\right| \leq c\left\|u_{p}\right\|_{\infty} t+\left|\log \left\|u_{\beta}\right\|_{\infty}^{c}\right| N_{t}^{\beta}$ and the equality

$$
\int_{\Omega_{0}} \exp \left(\sum_{\beta=1}^{p-1} r_{\beta} N_{t}^{\beta}\right) d \mu=\exp \left(\sum_{\beta=1}^{p-1}\left(e^{r_{\beta}}-1\right)\right)
$$

for $r_{\beta} \in \mathbb{R}$, we have for $c>0$,

$$
\left|\mathbb{E}_{P \times \mu}^{x, \alpha}\left[e^{c \mathrm{~S}_{\mathrm{spin}}}\right]\right| \leq \exp \left(t\left(c\left\|u_{p}\right\|_{\infty}+\sum_{\beta=1}^{p-1}\left(\left\|u_{\beta}\right\|_{\infty}^{c}-1\right)\right)\right),
$$

where $u_{\beta}$ is given in (5.16), and $\sup _{x} \mathbb{E}_{P}^{x}\left[e^{4 \mathrm{~S}_{V}}\right]<\infty$. Denote

$Z_{[v, w]}=-i \int_{v}^{w} a\left(B_{s}\right) \circ d B_{s}-\int_{v}^{w} U\left(B_{s}, \theta_{N_{s}}\right) d s+\sum_{\beta=1}^{p-1} \int_{v}^{w+} \log \left(-U_{\beta}\left(B_{s}, \theta_{N_{s-}}\right)\right) d N_{s}^{\beta}$

and let

$$
\mathrm{P}_{t} g\left(x, \theta_{\alpha}\right)=\mathbb{E}_{P \times \mu}^{x, \alpha}\left[e^{Z_{[0, t]}} g\left(B_{t}, \theta_{N_{t}}\right)\right] .
$$

Let $g \in \ell^{2}\left(\mathbb{Z}_{p}\right) \otimes C_{0}^{\infty}\left(\mathbb{R}^{d}\right)$. By the Schwarz inequality and setting $c=2$ in (5.29) we have the estimate

$$
\begin{aligned}
\left\|\mathrm{P}_{t} g\right\|^{2} & \leq \sum_{\alpha=1}^{p} \int_{\mathbb{R}^{d}} d x \mathbb{E}_{P \times \mu}^{x, \alpha}\left[g\left(B_{t}, \theta_{N_{t}}\right)^{2}\right] \mathbb{E}_{P \times \mu}^{x, \alpha}\left[e^{2 \mathrm{~S}_{\mathrm{spin}}}\right] \\
& \leq \exp \left(t\left(2\left\|u_{p}\right\|_{\infty}+\sum_{\beta=1}^{p-1}\left(\left\|u_{\beta}\right\|_{\infty}^{2}-1\right)\right)\right)\|g\|^{2} .
\end{aligned}
$$

Thus $\mathrm{P}_{t}$ is bounded. We show now that $\left\{\mathrm{P}_{t}\right\}_{t \geq 0}$ is a $C_{0}$-semigroup with generator $-\left(h_{\mathbb{Z}_{p}}+p-1\right)$, i.e., (1) $\mathrm{P}_{0}=I$, (2) $\mathrm{P}_{s} \mathrm{P}_{t}=\mathrm{P}_{s+t}$, (3) $\mathrm{P}_{t} g$ is continuous in $t$ and (4) $\lim _{t \rightarrow 0} \frac{1}{t}\left(\mathrm{P}_{t} g-g\right)=-\left(h_{\mathbb{Z}_{p}}+(p-1)\right) g$ in strong sense. First, $(1)$ is trivial. To check $(2)$ notice that

$$
\mathrm{P}_{t} \mathrm{P}_{s} g\left(x, \theta_{\alpha}\right)=\mathbb{E}_{P \times \mu}^{x, \alpha}\left[e^{Z_{[0, t]}} \mathbb{E}_{P \times \mu}^{B_{t}, N_{t}}\left[e^{Z_{[0, s]}} g\left(B_{s}, \theta_{N_{s}}\right)\right]\right] .
$$

By the Markov property of $B_{t}$ we have

$$
\begin{aligned}
& (5.30)=\mathbb{E}_{P \times \mu}^{x, \alpha}\left[e^{Z_{[0, t]}} \exp \left(-i \int_{t}^{t+s} a\left(B_{r}\right) \circ d B_{r}\right)\right. \\
& \left.\mathbb{E}_{\mu}^{N_{t}}\left[\exp \left(-\int_{0}^{s} U\left(B_{t+r}, \theta_{N_{r}}\right) d r+\sum_{\beta=1}^{p-1} \int_{0}^{s+} \log \left(-U_{\beta}\left(B_{t+r-}, \theta_{N_{r-}}\right)\right) d N_{r}^{\beta}\right) g\left(B_{t+s}, \theta_{N_{s}}\right)\right]\right] .
\end{aligned}
$$


Furthermore the Markov property of $N_{t}$ yields that

$$
(\underline{5.31})=\mathbb{E}_{P \times \mu}^{x, \alpha}\left[e^{Z_{[0, t]}} e^{Z_{[t, t+s]}} g\left(B_{t+s}, \theta_{N_{t+s}}\right)\right]=\mathrm{P}_{s+t} g\left(x, \theta_{\alpha}\right) .
$$

This proves the semigroup property (2). Next we obtain the generator of $\mathrm{P}_{t}$. An application of the Itô formula (see Appendix A) yields that

$$
d N_{t}=\sum_{\beta=1}^{p-1} \int_{0}^{t+} \beta d N_{s}^{\beta}, \quad d \theta_{N_{t}}=\sum_{\beta=1}^{p-1}\left(\theta_{N_{t}+\beta}-\theta_{N_{t}}\right)
$$

and

$$
\begin{aligned}
d g\left(B_{t}, \theta_{N_{t}}\right)= & \int_{0}^{t} \nabla g\left(B_{s}, \theta_{N_{s}}\right) \cdot d B_{s}+\frac{1}{2} \int_{0}^{t} \Delta g\left(B_{s}, \theta_{N_{s}}\right) d s \\
& +\sum_{\beta=1}^{p-1} \int_{0}^{t+}\left(g\left(B_{s}, \theta_{N_{s}+\beta}\right)-g\left(B_{s}, \theta_{N_{s}}\right)\right) d N_{s}^{\beta} \\
d e^{Z_{[0, t]}}= & \int_{0}^{t} e^{Z_{[0, s]}}\left(-i a\left(B_{s}\right)\right) \cdot d B_{s}+\frac{1}{2} \int_{0}^{t} e^{Z_{[0, s]}}\left(-i \nabla \cdot a\left(B_{s}\right)-a\left(B_{s}\right)^{2}\right) d s \\
& -\int_{0}^{t} e^{Z_{[0, s]}} U\left(B_{s}, \theta_{N_{s}}\right) d s+\sum_{\beta=1}^{p-1} \int_{0}^{t+} e^{Z_{[0, s-]}}\left(e^{\log \left(-U_{\beta}\left(B_{s}, \theta_{N_{s-}}\right)\right)}-1\right) d N_{s}^{\beta} .
\end{aligned}
$$

The product formula (Appendix A) $d\left(e^{Z_{[0, t]}} g\right)=d e^{Z_{[0, t]}} \cdot g+e^{Z_{[0, t]}} \cdot d g+d e^{Z_{[0, t]}} \cdot d g$ furthermore gives

$$
\begin{aligned}
d\left(e^{\left.Z_{[0, t]} g\right)\left(B_{t},\right.}, \theta_{N_{t}}\right)=\int_{0}^{t} e^{Z_{[0, s]}}\left\{\frac{1}{2} \Delta g\left(B_{s}, \theta_{N_{s}}\right)-i a\left(B_{s}\right) \cdot \nabla g\left(B_{s}, \theta_{N_{s}}\right)\right. & \left.+\left(-\frac{1}{2} a\left(B_{s}\right)^{2}-U\left(B_{s}, \theta_{N_{s}}\right)\right) g\left(B_{s}, \theta_{N_{s}}\right)\right\} d s \\
& +\int_{0}^{t} e^{Z_{[0, s]}}\left(\nabla g\left(B_{s}, \theta_{N_{s}}\right)-i a\left(B_{s}\right) g\left(B_{s}, \theta_{N_{s}}\right)\right) \cdot d B_{s} \\
& +\sum_{\beta=1}^{p-1} \int_{0}^{t+} e^{Z_{[0, s]}}\left(g\left(B_{s}, \theta_{N_{s-}+\beta}\right) e^{\log \left(-U_{\beta}\left(B_{s}, \theta_{N_{s-}}\right)\right)}-g\left(B_{s}, \theta_{N_{s-}}\right)\right) d N_{s}^{\beta} .
\end{aligned}
$$

Taking expectation values on both sides above yields

$$
\frac{1}{t}\left(f,\left(\mathrm{P}_{t}-1\right) g\right)=\frac{1}{t} \int_{0}^{t} d s \int_{\mathbb{R}^{d}} d x \overline{f(x)} \mathbb{E}_{P \times \mu}^{x, \alpha}[G(s)]
$$


where

$$
\begin{aligned}
G(s)=e^{Z_{[0, s]}}\left(\frac{1}{2} \Delta-i a\left(B_{s}\right) \cdot \nabla-\frac{1}{2} a\left(B_{s}\right)^{2}-U\left(B_{s}, \theta_{N_{s}}\right)\right) g\left(B_{s}, \theta_{N_{s}}\right) & \\
& +\sum_{\beta=1}^{p-1} e^{Z_{[0, s]}}\left(g\left(B_{s}, \theta_{N_{s}+\beta}\right) e^{\log \left(-U_{\beta}\left(B_{s}, \theta_{N_{s}}\right)\right)}-g\left(B_{s}, \theta_{N_{s-}}\right)\right) \\
G(0)= & \left(\frac{1}{2} \Delta-i a\left(B_{0}\right) \cdot \nabla-\frac{1}{2} a\left(B_{0}\right)^{2}-U\left(B_{0}, \theta_{N_{0}}\right)\right) g\left(B_{0}, \theta_{N_{0}}\right) \\
& +\sum_{\beta=1}^{p-1}\left(-U_{\beta}\left(B_{0}, \theta_{N_{0}}\right) g\left(B_{0}, \theta_{N_{0}+\beta}\right)-g\left(B_{0}, \theta_{N_{0}}\right)\right) \\
= & -\left(h_{\mathbb{Z}_{p}}+(p-1)\right) g\left(x, \theta_{\alpha}\right) .
\end{aligned}
$$

Note that $U(x, \theta), U_{\beta}(x, \theta), a_{\mu}(x)$ are continuous in $x$. Therefore $G(s)$ is continuous at $s=0$ for each $(\omega, \tau) \in \Omega_{P} \times \Omega_{N}$, and $\mathbb{E}_{P \times \mu}^{x, \alpha}[G(s)]$ is continuous at $s=0$ by the dominated convergence theorem. Thus

$$
\lim _{t \rightarrow 0} \frac{1}{t}\left(f,\left(\mathrm{P}_{t}-1\right) g\right)=\left(f,-\left(h_{\mathbb{Z}_{p}}+(p-1)\right) g\right)
$$

follows. Finally, the strong continuity (3) follows from (2) and (4), and hence

$$
e^{t(p-1)} \mathrm{P}_{t} g=e^{-t h_{\mathbb{Z}_{p}}} g
$$

By a similar approximation argument as in the proof of Proposition 3.7, (5.32) can be extended to a obeying Assumption (A2). Finally, we extend (5.32) for $U$ and $U_{\beta}$ given in Definition 5.3. By using a mollifier it is seen that there exists a sequence $U_{\beta}^{(n)}\left(x, \theta_{\alpha}\right)$ and $U^{(n)}\left(x, \theta_{\alpha}\right), n=1,2,3, \ldots$, such that they are continuous in $x$ and converge to $U_{\beta}\left(x, \theta_{\alpha}\right)$ resp. $U\left(x, \theta_{\alpha}\right)$ for each $x$ as $n \rightarrow \infty$, and $\left\|U^{(n)}\left(\cdot, \theta_{\alpha}\right)\right\|_{\infty} \leq\left\|U\left(\cdot, \theta_{\alpha}\right)\right\|_{\infty}$ and $\left\|U_{\beta}^{(n)}\left(\cdot, \theta_{\alpha}\right)\right\|_{\infty} \leq\left\|U_{\beta}\left(\cdot, \theta_{\alpha}\right)\right\|_{\infty}$. For each fixed $\tau \in \Omega_{N}$ there exists $r_{1}=r_{1}(\tau), \ldots, r_{M}=$ $r_{M}(\tau)$, where $M=M(\tau)$, such that

$$
\exp \left(\sum_{\beta=1}^{p-1} \int_{0}^{t+} \log \left(-U_{\beta}\left(B_{s}, \theta_{N_{s-}}\right)\right) d N_{s}^{\beta}\right)=\prod_{\beta=1}^{p-1} \prod_{i=1}^{M}\left(-U_{\beta}\left(B_{r_{i}}, \theta_{N_{r_{i}}}\right)\right) .
$$

Then for each $\tau \in \Omega_{N}$,

$$
\begin{array}{r}
\lim _{n \rightarrow \infty} \exp \left(\sum_{\beta=1}^{p-1} \int_{0}^{t+} \log \left(-U_{\beta}^{(n)}\left(B_{s}, \theta_{N_{s-}}\right)\right) d N_{s}^{\beta}\right) \\
=\exp \left(\sum_{\beta=1}^{p-1} \int_{0}^{t+} \log \left(-U_{\beta}\left(B_{s}, \theta_{N_{s-}}\right)\right) d N_{s}^{\beta}\right) .
\end{array}
$$


In the same way as above we can also see that $e^{-\int_{0}^{t} U^{(n)}\left(B_{s}, \theta_{N_{s}}\right) d s} \rightarrow e^{-\int_{0}^{t} U\left(B_{s}, \theta_{N_{s}}\right) d s}$ as $n \rightarrow \infty$ almost surely. Therefore by the dominated convergence theorem (5.32) holds for such $U_{\beta}$ and $U$.

Now we can state and prove the functional integral representation of $e^{-t H_{\mathbb{Z}_{p}}^{\Psi}}$.

Theorem 5.11 Let $\Psi \in \mathscr{B}_{0}$, and $U, U_{\beta}$ be given as in Definition 5.3. Assume $u_{\beta} \in$ $L^{\infty}\left(\mathbb{R}^{d}\right), \beta=1, \ldots, p, V \in L^{\infty}\left(\mathbb{R}^{d}\right)$, and let Assumption (A2) and

$$
\int_{\mathbb{R}} \rho(r, t) d r \int_{0}^{r} d s \int_{\mathbb{R}^{d}} d y(2 \pi s)^{-d / 2} e^{-|x-y|^{2} /(2 s)}\left|\log u_{\beta}(y)\right|<\infty, \quad \beta=1, \ldots, p-1,
$$

where $\rho(r, t)$ is the distribution of $T_{t}^{\Psi}$ on $\mathbb{R}$. Then

$$
\left(f, e^{-t H_{\mathbb{Z}_{p}}^{\Psi}} g\right)=\sum_{\alpha=1}^{p} \int_{\mathbb{R}^{d}} d x \mathbb{E}_{P \times \mu \times \nu}^{x, \alpha, 0}\left[e^{(p-1) T_{t}^{\Psi}} \overline{f\left(B_{0}, \theta_{N_{0}}\right)} g\left(B_{T_{t}^{\Psi}}, \theta_{N_{T_{t}^{\Psi}}}\right) e^{\mathrm{S}^{\Psi}}\right],
$$

where $\mathrm{S}^{\Psi}=\mathrm{S}_{V}^{\Psi}+\mathrm{S}_{a}^{\Psi}+\mathrm{S}_{\text {spin }}^{\Psi}$ and

$$
\begin{aligned}
& \mathrm{S}_{V}^{\Psi}=-\int_{0}^{t} V\left(B_{T_{s}^{\Psi}}\right) d s \\
& \mathrm{~S}_{a}^{\Psi}=-i \int_{0}^{T_{t}^{\Psi}} a\left(B_{s}\right) \circ d B_{s} \\
& \mathrm{~S}_{\mathrm{spin}}^{\Psi}= \begin{cases}-\int_{0}^{T_{t}^{\Psi}}\left(U\left(B_{s}, \theta_{N_{s}}\right)-\mathscr{E}_{h_{\mathbb{Z}_{p}}}\right) d s+\sum_{\beta=1}^{p-1} \int_{0}^{T_{t}^{\Psi}+} \log \left(-U_{\beta}\left(B_{s}, \theta_{N_{s-}}\right)\right) d N_{s}^{\beta} \\
\text { if } \mathscr{E}_{h_{\mathbb{Z}_{p}}}<0, \\
-\int_{0}^{T_{t}^{\Psi}}\left(U\left(B_{s}, \theta_{N_{s}}\right)\right) d s+\sum_{\beta=1}^{p-1} \int_{0}^{T_{t}^{\Psi}+} \log \left(-U_{\beta}\left(B_{s}, \theta_{N_{s-}}\right)\right) d N_{s}^{\beta} \\
\text { if } \mathscr{E}_{h_{\mathbb{Z}_{p}}} \geq 0 .\end{cases}
\end{aligned}
$$

Proof. Since from (5.35) it follows that

$$
\begin{aligned}
& \mathbb{E}_{P \times \mu \times \nu}^{x, \alpha, 0}\left[\int_{0}^{T_{t}^{\Psi}+}\left|\log \left(-U_{\beta}\left(B_{s}, \theta_{N_{s-}}\right)\right)\right| d N_{s}^{\beta}\right] \\
& \leq \int_{\mathbb{R}} \rho(r, t) d r \int_{0}^{r} d s \int_{\mathbb{R}^{d}} \frac{e^{-|x-y|^{2} /(2 s)}}{(2 \pi s)^{d / 2}}\left|\log u_{\beta}(y)\right|<\infty
\end{aligned}
$$

we notice that

$$
\int_{0}^{T_{t}^{\Psi}+}\left|\log \left(-U_{\beta}\left(B_{s}, \theta_{N_{s-}}\right)\right)\right| d N_{s}^{\beta}<\infty
$$


almost surely. Using Lemma 5.10 we obtain

$$
\left(f, e^{-t \Psi\left(\overline{h_{\mathbb{Z}_{p}}}\right)} g\right)=\sum_{\alpha=1}^{p} \int_{\mathbb{R}^{d}} d x \mathbb{E}_{P \times \mu \times \nu}^{x, \alpha, 0}\left[e^{(p-1) T_{t}^{\Psi}} \overline{f\left(B_{0}, \theta_{N_{0}}\right)} g\left(B_{T_{t}^{\Psi}}, \theta_{N_{T_{t}}}\right) e^{\mathrm{S}_{a}^{\Psi}+\mathrm{S}_{\mathrm{spin}}^{\Psi}}\right] .
$$

Let $0=t_{0}<t_{1}<\cdots<t_{n}=t$. We show that

$$
\begin{aligned}
& \left(f_{0}, \prod_{j=1}^{n} e^{-\left(t_{j}-t_{j-1}\right) \Psi\left(\overline{h_{\mathbb{Z}_{p}}}\right)} f_{j}\right) \\
& =\sum_{\alpha=1}^{p} \int_{\mathbb{R}^{d}} d x \mathbb{E}_{P \times \mu \times \nu}^{x, \alpha, 0}\left[e^{(p-1) T_{t}^{\Psi}} \overline{f\left(B_{0}, \theta_{N_{0}}\right)}\left(\prod_{j=1}^{n} f_{j}\left(B_{T_{t_{j}}^{\Psi}}, \theta_{N_{T_{t_{j}}^{\Psi}}}\right)\right) e^{\mathrm{S}_{a}^{\Psi}+\mathrm{S}_{\mathrm{spin}}^{\Psi}}\right] .
\end{aligned}
$$

This can be proven in the same way as in Step 2 of the proof of Theorem 3.16 with the $d$-dimensional Brownian motion $B_{t}$ on $\left(\Omega_{P}, \mathscr{F}_{P}, P^{x}\right)$ replaced by the $d+1$ dimensional Markov process $\left(B_{t}, N_{t}\right)$ on $\left(\Omega_{P} \times \Omega_{N}, \mathscr{F}_{P} \times \mathscr{F}_{N}, P^{x} \times \mu\right)$ under the natural filtration. Suppose $V$ is continuous. By the Trotter product formula and (5.39) it is seen that

$$
\begin{aligned}
& \left(f, e^{-t H_{\mathbb{Z}_{p}}^{\Psi}} g\right)=\lim _{n \rightarrow \infty}\left(f,\left(e^{-(t / n) \Psi\left(h_{\mathbb{Z}_{p}}\right)} e^{-(t / n) V}\right)^{n} g\right) \\
& =\lim _{n \rightarrow \infty} \sum_{\alpha=1}^{p} \int_{\mathbb{R}^{d}} d x \mathbb{E}_{P \times \mu \times \nu}^{x, \alpha, 0}
\end{aligned}
$$

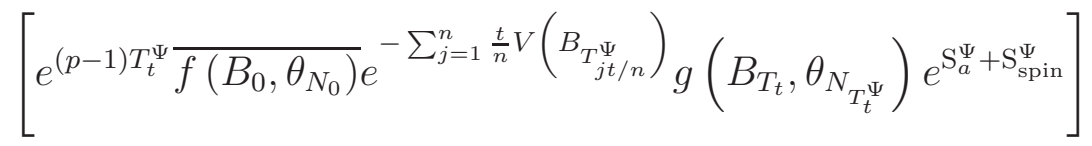

$$
\begin{aligned}
& =\sum_{\alpha=1}^{p} \int_{\mathbb{R}^{d}} d x \mathbb{E}_{P \times \mu \times \nu}^{x, \alpha, 0}\left[e^{(p-1) T_{t}^{\Psi}} \overline{f\left(B_{0}, \theta_{N_{0}}\right)} g\left(B_{T_{t}^{\Psi}}, \theta_{N_{T_{t}^{\Psi}}}\right) e^{\mathrm{S}^{\Psi}}\right] .
\end{aligned}
$$

Hence the theorem follows for continuous $V$. This can be extended for $V \in L^{\infty}\left(\mathbb{R}^{d}\right)$ in the same way as in Step 4 of the proof of Theorem 3.8 ,

qed

In the case of $\Psi(u)=\sqrt{2 u+m^{2}}-m$, the distribution of $T_{t}^{\Psi}$ is exactly given by (2.4).

Remark 5.12 Notice that conditions (5.26) and (5.35) depend on $t$. Let us replace (5.26) and (5.35) with the condition

$$
\int_{0}^{\infty} d s \int_{\mathbb{R}^{d}} d y(2 \pi s)^{-d / 2} e^{-|x-y|^{2} /(2 s)}\left|\log u_{\beta}(y)\right|<\infty, \quad \beta=1, \ldots, p-1 .
$$

Then we see that

$$
\mathbb{E}_{P \times \mu \times \nu}^{x, \alpha, 0}\left[\int_{0}^{T_{t}^{\Psi}+}\left|\log \left(-U_{\beta}\left(B_{s}, \theta_{N_{s-}}\right)\right)\right| d N_{s}^{\beta}\right] \leq \int_{0}^{\infty} d s \int_{\mathbb{R}^{d}} \frac{e^{-|x-y|^{2} /(2 s)}}{(2 \pi s)^{d / 2}}\left|\log u_{\beta}(y)\right|<\infty
$$


and

$$
\mathbb{E}_{P \times \mu}^{x, \alpha}\left[\int_{0}^{t+}\left|\log \left(-U_{\beta}\left(B_{s}, \theta_{N_{s-}}\right)\right)\right| d N_{s}^{\beta}\right] \leq \int_{0}^{\infty} d s \int_{\mathbb{R}^{d}} \frac{e^{-|x-y|^{2} /(2 s)}}{(2 \pi s)^{d / 2}}\left|\log u_{\beta}(y)\right|<\infty .
$$

In particular

$$
\int_{0}^{T_{t}^{\Psi}+}\left|\log \left(-U_{\beta}\left(B_{s}, \theta_{N_{s-}}\right)\right)\right| d N_{s}^{\beta}<\infty
$$

and

$$
\int_{0}^{t+}\left|\log \left(-U_{\beta}\left(B_{s}, \theta_{N_{s-}}\right)\right)\right| d N_{s}^{\beta}<\infty
$$

follow for all $t \geq 0$.

Now let $h_{\mathbb{Z}_{p}}^{0}$ be defined by $h_{\mathbb{Z}_{p}}$ in (5.19) with $a$ and $U_{\beta}, \beta=1, \ldots, p-1$, replaced by 0 and $\left|U_{\beta}\right|$, respectively, i.e.,

$$
\left(h_{\mathbb{Z}_{p}}^{0} f\right)\left(x, \theta_{\alpha}\right)=\frac{1}{2} \mathrm{p}^{2} f\left(x, \theta_{\alpha}\right)+U\left(x, \theta_{\alpha}\right) f\left(x, \theta_{\alpha}\right)-\sum_{\beta=1}^{p-1}\left|U_{\beta}(x, \sigma)\right| f\left(x, \theta_{\alpha+\beta}\right) .
$$

Let

$$
\overline{h_{\mathbb{Z}_{p}}^{0}}= \begin{cases}h_{\mathbb{Z}_{p}}^{0} & \text { if } \mathscr{E}_{h_{\mathbb{Z}_{p}}^{0}} \geq 0, \\ h_{\mathbb{Z}_{p}}^{0}-\mathscr{E}_{h_{\mathbb{Z}_{p}}^{0}} & \text { if } \mathscr{E}_{h_{\mathbb{Z}_{p}}^{0}}<0 .\end{cases}
$$

An immediate corollary of Theorem 5.11 is

Corollary 5.13 (Diamagnetic inequality) Under the assumptions of Theorem 5.11 we have

$$
h_{\mathbb{Z}_{p}}-\mathscr{E}_{h_{\mathbb{Z}_{p}}^{0}} \geq 0
$$

Moreover,

(1) if $\mathscr{E}_{h_{\mathbb{Z}_{p}}^{0}} \geq 0$, then

$$
\left|\left(f, e^{-t\left(\Psi\left(h_{\mathbb{Z}_{p}}\right)+V\right)} g\right)\right| \leq\left(|f|, e^{-t\left(\Psi\left(\overline{h_{\mathbb{Z}_{p}}^{0}}\right)+V\right)}|g|\right)
$$

and

$$
\mathscr{E}_{\Psi\left(\overline{h_{\mathbb{Z}_{p}}^{0}}\right)+V} \leq \mathscr{E}_{\Psi\left(h_{\mathbb{Z}_{p}}\right)+V}
$$


(2) if $\mathscr{E}_{h_{\mathbb{Z}_{p}}^{0}}<0$, then

$$
\left|\left(f, e^{-t\left(\Psi\left(h_{\mathbb{Z}_{p}}-\mathscr{E}_{h_{\mathbb{Z}_{p}}^{0}}\right)+V\right)} g\right)\right| \leq\left(|f|, e^{-t\left(\Psi\left(\overline{h_{\mathbb{Z}_{p}}^{0}}\right)+V\right)}|g|\right)
$$

and

$$
\left.\mathscr{E}_{\Psi}\left(\overline{h_{\mathbb{Z}_{p}}^{0}}\right)+V=\mathscr{E}_{\Psi\left(h_{\mathbb{Z}_{p}}-\mathscr{E}_{h_{\mathbb{Z}_{p}}^{0}}\right.}\right)+V
$$

Proof. Note the estimate

$$
\left|\exp \left(\sum_{\beta=1}^{p-1} \int_{0}^{T_{t}^{\Psi}+} \log \left(-U_{\beta}\left(\theta_{N_{s-}^{\beta}}\right)\right) d N_{s}^{\beta}\right)\right| \leq \exp \left(\sum_{\beta=1}^{p-1} \int_{0}^{T_{t}^{\Psi}+} \log \left|U_{\beta}\left(\theta_{N_{s-}^{\beta}}\right)\right| d N_{s}^{\beta}\right) .
$$

Let $\Psi(u)=u$ and then $T_{t}^{\Psi}=t$. Theorem 5.11 and (5.50) imply that

$$
\left|\left(f, e^{-t h_{\mathbb{Z}_{p}}} g\right)\right| \leq\left(|f|, e^{-t h_{\mathbb{Z}_{p}}^{0}}|g|\right) .
$$

This further implies $\mathscr{E}_{h_{\mathbb{Z}_{p}}^{0}} \leq \mathscr{E}_{h_{\mathbb{Z}_{p}}}$, thus (5.45) holds. (5.46) and (5.48) follow similarly by Theorem 5.11 and the estimate (5.50). (5.47) and (5.49) are an immediate consequence of (5.46) and (5.48), respectively.

Theorem 5.14 Let $U$ and $U_{\beta}$ be given by Definition 5.3 and suppose that $\sum_{\beta=1}^{p} c_{\beta}<1$. Let Assumption (A2) and (5.26) hold, and suppose that $|V|$ is relatively bounded with respect to $\Psi\left(\overline{h_{\mathbb{Z}_{p}}^{0}}\right)$ with a relative bound $b$. Then $|V|$ is relatively bounded with respect to $\Psi\left(\overline{h_{\mathbb{Z}_{p}}}\right)$ with a relative bound not larger than $b$.

ProOF. We prove the theorem in the case of $\mathscr{E}_{h_{\mathbb{Z}_{p}}^{0}}<0$, the case $\mathscr{E}_{h_{\mathbb{Z}_{p}}^{0}} \geq 0$ is simpler. By the assumption we have for every $\epsilon>0$,

$$
\|V f\| \leq(b+\epsilon)\left\|\Psi\left(\overline{h_{\mathbb{Z}_{p}}^{0}}\right) f\right\|+c\|f\| .
$$

By virtue of Corollary 5.13 we have

$$
\frac{\left\||V|\left(\Psi\left(h_{\mathbb{Z}_{p}}-\mathscr{E}_{h_{\mathbb{Z}_{p}}^{0}}\right)+E\right)^{-1} f\right\|}{\|f\|} \leq \frac{\left\||V|\left(\Psi\left(\overline{h_{\mathbb{Z}_{p}}^{0}}\right)+E\right)^{-1}|f|\right\|}{\|f\|}
$$

By (5.52) the right hand side of (5.53) converges to a number smaller than $b+\epsilon$ as $E \rightarrow \infty$. Thus

$$
\|V f\| \leq(b+\epsilon)\left\|\Psi\left(h_{\mathbb{Z}_{p}}-\mathscr{E}_{h_{\mathbb{Z}_{p}}^{0}}\right) f\right\|+c_{b}\|f\|
$$


follows with some constant $c_{b}$. Let $X<Y$ and $X<0$. From (2.1) we can see that

$$
\Psi(u-X)-\Psi(u-Y)=b(Y-X)+\int_{0}^{\infty} e^{-(u-Y) y}\left(1-e^{-(Y-X) y}\right) \lambda(d y), \quad u \geq Y
$$

Hence $\sup _{u \geq Y}|\Psi(u-X)-\Psi(u-Y)| \leq \Psi(Y-X)$. From this and $\mathscr{E}_{h_{\mathbb{Z}_{p}}^{0}} \leq \mathscr{E}_{h_{\mathbb{Z}_{p}}}$ we obtain that

$$
\sup _{u \geq \mathscr{E}_{\mathfrak{Z}_{p}}}\left|\Psi\left(u-\mathscr{E}_{h_{\mathbb{Z}_{p}}^{0}}\right)-\Psi\left(u-\mathscr{E}_{h_{\mathbb{Z}_{p}}}\right)\right| \leq \Psi\left(\mathscr{E}_{h_{\mathbb{Z}_{p}}}-\mathscr{E}_{h_{\mathbb{Z}_{p}}^{0}}\right)
$$

Thus the spectral decomposition yields that

$$
\left\|\Psi\left(h_{\mathbb{Z}_{p}}-\mathscr{E}_{h_{\mathbb{Z}_{p}}^{0}}\right) f\right\| \leq\left\|\Psi\left(h_{\mathbb{Z}_{p}}-\mathscr{E}_{h_{\mathbb{Z}_{p}}}\right) f\right\|+\Psi\left(\mathscr{E}_{h_{\mathbb{Z}_{p}}}-\mathscr{E}_{h_{\mathbb{Z}_{p}}^{0}}\right)\|f\|
$$

Then the theorem follows together with (5.54), since $\epsilon$ is arbitrary.

We have the immediate consequences below.

Theorem 5.15 Let $U$ and $U_{\beta}$ be given in Definition 5.3 and assume $\sum_{\beta=1}^{p} c_{\beta}<1$. Suppose that $V$ is relatively bounded with respect to $\Psi\left(\overline{h_{\mathbb{Z}_{p}}^{0}}\right)$ with a relative bound strictly less than 1. Moreover, assume (5.26).

(1) Let Assumption (A2) hold. Then $H_{\mathbb{Z}_{p}}^{\Psi}$ is self-adjoint on $D\left(\Psi\left(\overline{h_{\mathbb{Z}_{p}}}\right)\right)$ and essentially self-adjoint on any core of $\Psi\left(\overline{h_{\mathbb{Z}_{p}}}\right)$. In particular, under Assumption (A3) the operator $H_{\mathbb{Z}_{p}}^{\Psi}$ is essentially self-adjoint on $C_{0}^{\infty}\left(\mathbb{R}^{d}\right)$.

(2) Let Assumption (A3) hold. Then the functional integral representation of $e^{-t H_{\mathbb{Z}_{p}}^{\Psi}}$ is given by (5.11).

Proof. (1) is trivial. (2) Let $V=V_{+}-V_{-}$. Note that $V_{+}, V_{-}$are relatively bounded with respect to $\Psi\left(\mathrm{p}^{2} / 2\right)$ with a relative bound strictly less than 1 . Define $V_{+, n}(x)=$ $\phi(x / n)\left(V_{+} * j_{n}\right)$ and $V_{-, m}=\phi(x / n)\left(V_{-} * j_{m}\right)$, where $\phi$ and $j_{n}$ are defined in Step 4 of the proof of Theorem 3.16. Notice that $e^{-t\left(\Psi\left(\overline{h_{\mathbb{Z}_{p}}}\right)+V_{+, n}-V_{-, m}\right)}$ strongly converges to $e^{-t\left(\Psi\left(\overline{h_{\mathbb{Z}_{p}}}\right)+V\right)}$ as $n, m \rightarrow \infty$, since $\Psi\left(\overline{h_{\mathbb{Z}_{p}}}\right)+V_{+, n}-V_{-, m}$ converges to $\Psi\left(\overline{h_{\mathbb{Z}_{p}}}\right)+V$ on the common core $\ell^{2}\left(\mathbb{Z}_{2}\right) \otimes C_{0}^{\infty}\left(\mathbb{R}^{3}\right)$. Then the theorem can be proven in a similar way to Step 4 of Theorem 3.16. 


\section{Relativistic Schrödinger operators}

\subsection{Case of spin $1 / 2$}

In this subsection we further discuss the functional integral representation for the specific case of the relativistic Schrödinger operator with spin $1 / 2$. Throughout this section $d=3$ and $p=2$. Therefore, $\theta_{\alpha}=\theta_{\alpha}^{(2)}, \alpha=1,2$, and $\theta_{1}=-1$ and $\theta_{2}=+1$. The relativistic Schrödinger operator with spin $1 / 2$ is given by

$$
h_{1 / 2}^{\mathrm{rel}}=\sqrt{2 h_{1 / 2}+m^{2}}-m, \quad m \geq 0,
$$

on $L^{2}\left(\mathbb{R}^{3} ; \mathbb{C}^{2}\right)$, where $h_{1 / 2}=(\sigma \cdot(\mathrm{p}-a))^{2}$.

Functional integral representation. Let Assumption (A4) hold. Then $h_{1 / 2}^{\text {rel }}$ is unitary equivalent to

$$
h_{\mathbb{Z}_{2}}^{\mathrm{rel}}=\sqrt{2 h_{\mathbb{Z}_{2}}+m^{2}}-m,
$$

where $h_{\mathbb{Z}_{2}}$ is defined on $L^{2}\left(\mathbb{R}^{3} \times \mathbb{Z}_{2}\right)$ and given in (5.7) as

$$
\left(h_{\mathbb{Z}_{2}} f\right)(x, \theta):=\left(\frac{1}{2}(\mathrm{p}-a)^{2} f\right)(x, \theta)-\frac{1}{2} \theta b_{3}(x) f(x, \theta)-\frac{1}{2}\left(b_{1}(x)-i \theta b_{2}(x)\right) f(x,-\theta)
$$

for $x \in \mathbb{R}^{3}$ and $\theta \in \mathbb{Z}_{2}$. Recall that here $b=\left(b_{1}, b_{2}, b_{3}\right)=\nabla \times a$. Clearly, $h_{\mathbb{Z}_{2}}^{\text {rel }}$ is non-negative and $h_{\mathbb{Z}_{2}}^{\text {rel }}=\Psi\left(h_{\mathbb{Z}_{2}}\right)$ with the Bernstein function $\Psi(u)=\sqrt{2 u+m^{2}}-m$. The spin operator in $h_{\mathbb{Z}_{2}}$ is furthermore given by

$$
\begin{array}{ll}
\text { (diagonal component) } & U(x, \theta)=-\frac{1}{2} \theta b_{3}(x), \\
\text { (off - diagonal component) } & U_{1}(x, \theta)=-\frac{1}{2}\left(b_{1}(x)-i \theta b_{2}(x)\right) .
\end{array}
$$

Let $h_{\mathbb{Z}_{2}}^{0}$ be defined by $h_{\mathbb{Z}_{2}}$ with vector potential $a \in\left(L_{\text {loc }}^{4}\left(\mathbb{R}^{3}\right)\right)^{3}$ and off-diagonal component $U_{1}$ replaced by 0 and $\left|U_{1}\right|=\frac{1}{2} \sqrt{b_{1}^{2}(x)+b_{2}^{2}(x)}$, respectively, i.e.,

$$
\left(h_{\mathbb{Z}_{2}}^{0} f\right)(x, \theta)=\left(\frac{1}{2} \mathrm{p}^{2} f\right)(x, \theta)-\frac{1}{2} \theta b_{3}(x) f(x, \theta)-\frac{1}{2} \sqrt{b_{1}(x)^{2}+b_{2}(x)^{2}} f(x,-\theta) .
$$

The operator $h_{\mathbb{Z}_{2}}^{0}$ is unitary equivalent with $h_{1 / 2}^{0}$ on $L^{2}\left(\mathbb{R}^{3} ; \mathbb{C}^{2}\right)$ given by

$$
h_{1 / 2}^{0}=\frac{1}{2} \mathrm{p}^{2}-\frac{1}{2}\left[\begin{array}{cc}
b_{3} & \sqrt{b_{1}^{2}+b_{2}^{2}} \\
\sqrt{b_{1}^{2}+b_{2}^{2}} & -b_{3}
\end{array}\right] .
$$


We write

$$
\begin{aligned}
& h_{\mathbb{Z}_{2}}^{\mathrm{rel}}(0)=\sqrt{2 \overline{h_{\mathbb{Z}_{2}}^{0}}+m^{2}}-m, \\
& h_{1 / 2}^{\mathrm{rel}}(0)=\sqrt{2 \overline{h_{1 / 2}^{0}}+m^{2}}-m,
\end{aligned}
$$

where $\overline{h_{\mathbb{Z}_{2}}^{0}}=h_{\mathbb{Z}_{2}}^{0}-\mathscr{E}_{h_{\mathbb{Z}_{2}}^{0}}$ and $\overline{h_{1 / 2}^{0}}=h_{1 / 2}^{0}-\mathscr{E}_{h_{1 / 2}^{0}}$. The operators $h_{1 / 2}^{\text {rel }}$ and $h_{\mathbb{Z}_{2}}^{\text {rel }}$ are essentially self-adjoint on $\mathbb{C}^{2} \otimes C_{0}^{\infty}\left(\mathbb{R}^{3}\right)$ and $\ell^{2}\left(\mathbb{Z}_{2}\right) \otimes C_{0}^{\infty}\left(\mathbb{R}^{3}\right)$, respectively.

Theorem 6.1 Let Assumption (A4) hold and further assume (1)-(4) below:

(1) $V$ is relatively bounded with respect to $\sqrt{\mathrm{p}^{2}+m^{2}}$ with a relative bound $A<1$;

(2) each $-\frac{1}{2} b_{j}, j=1,2,3$, is relatively bounded with respect to $\frac{1}{2} \mathrm{p}^{2}$ with a relative bound $\kappa_{j} \geq 0$;

(3) $A\left(1-\left(\kappa_{1}+\kappa_{2}+\kappa_{3}\right)\right)^{-1 / 2}<1$;

(4) $\int_{\mathbb{R}^{3}} \frac{\left|\log \left(\frac{1}{2} \sqrt{b_{1}(y)^{2}+b_{2}(y)^{2}}\right)\right|}{2 \pi|x-y|} d y<\infty$, a.e. $x \in \mathbb{R}^{3}$.

Then the relativistic Schrödinger operator $h_{1 / 2}^{\mathrm{rel}}+V\left(\right.$ resp. $\left.h_{\mathbb{Z}_{2}}^{\mathrm{rel}}+V\right)$ is essentially selfadjoint on $\mathbb{C}^{2} \otimes C_{0}^{\infty}\left(\mathbb{R}^{3}\right)$ (resp. $\left.\ell^{2}\left(\mathbb{Z}_{2}\right) \otimes C_{0}^{\infty}\left(\mathbb{R}^{3}\right)\right)$ and

$$
\left(f, e^{-t\left(h_{\mathbb{Z}_{2}}^{\mathrm{rel}}+V\right)} g\right)=\sum_{\alpha=1,2} \int_{\mathbb{R}^{3}} d x \mathbb{E}_{P \times \mu \times \nu}^{x, \alpha, 0}\left[e^{T_{t}^{\Psi}} \overline{f\left(B_{0}, \theta_{N_{0}}\right)} g\left(B_{T_{t}^{\Psi}}, \theta_{N_{T_{t}^{\Psi}}}\right) e^{\mathrm{S}^{\Psi}}\right],
$$

where $\theta_{N_{T_{t}}}=(-1)^{N_{T_{t}}}$, the subordinator $T_{t}^{\Psi}$ is defined by $T_{t}^{\Psi}=\inf \left\{s>0 \mid B_{s}+m s=t\right\}$ and the exponent $\mathrm{S}^{\Psi}=\mathrm{S}_{V}^{\Psi}+\mathrm{S}_{a}^{\Psi}+\mathrm{S}_{\text {spin }}^{\Psi}$ is given by

$$
\begin{aligned}
& \mathrm{S}_{V}^{\Psi}=-\int_{0}^{t} V\left(B_{T_{s}^{\Psi}}\right) d s \\
& \mathrm{~S}_{a}^{\Psi}=-i \int_{0}^{T_{t}^{\Psi}} a\left(B_{s}\right) \circ d B_{s}, \\
& \mathrm{~S}_{\mathrm{spin}}^{\Psi}=\int_{0}^{T_{t}^{\Psi}} \frac{1}{2} b_{3}\left(B_{s}\right) \theta_{N_{s}} d s+\int_{0}^{T_{t}^{\Psi}+} \log \left(\frac{1}{2}\left(b_{1}\left(B_{s}\right)-i \theta_{N_{s-}} b_{2}\left(B_{s}\right)\right)\right) d N_{s} .
\end{aligned}
$$

Proof. Set $S=-\frac{1}{2}\left[\begin{array}{cc}b_{3} & \sqrt{b_{1}^{2}+b_{2}^{2}} \\ \sqrt{b_{1}^{2}+b_{2}^{2}} & -b_{3}\end{array}\right]$. We see that $S$ is relatively bounded with respect to $\frac{1}{2} \mathrm{p}^{2}\left[\begin{array}{ll}1 & 0 \\ 0 & 1\end{array}\right]$ with a relative bound $\kappa=\kappa_{1}+\kappa_{2}+\kappa_{3}$. Note that

$$
\left\|\sqrt{\mathrm{p}^{2}+m^{2}} f\right\|^{2}=\left(f,\left(\mathrm{p}^{2}+m^{2}\right) f\right)=\left\|\left(h_{1 / 2}^{\mathrm{rel}}(0)+m\right) f\right\|^{2}+2\left(f,-S f+\mathscr{E}_{h_{1 / 2}^{0}} f\right) .
$$


Since $|(f, S f)| \leq \kappa\left(f, \frac{1}{2} \mathrm{p}^{2} f\right)+\kappa^{\prime}\|f\|^{2}$, with a constant $\kappa^{\prime}$, we have

$$
\left\|\sqrt{\mathrm{p}^{2}+m^{2}} f\right\|^{2} \leq\left\|\left(h_{1 / 2}^{\mathrm{rel}}(0)+m\right) f\right\|^{2}+\kappa\left\|\sqrt{\mathrm{p}^{2}+m^{2}} f\right\|^{2}+\left(\left|\mathscr{E}_{h_{1 / 2}^{0}}\right|+\kappa^{\prime}\right)\|f\| .
$$

Together with $\|V f\| \leq A\left\|\sqrt{\mathrm{p}^{2}+m^{2}} f\right\|+A^{\prime}\|f\|^{2}$, with a constant $A^{\prime}$, we have

$$
\|V f\| \leq A(1-\kappa)^{-1 / 2}\left\|h_{1 / 2}^{\mathrm{rel}}(0) f\right\|+\left(A^{\prime}+A m+A \sqrt{2\left|\mathscr{E}_{h_{1 / 2}^{0}}\right|+\kappa^{\prime}}\right)\|f\| .
$$

Thus by assumption (3) above, $V$ is relatively bounded with respect to $h_{1 / 2}^{\text {rel }}(0)$ with relative bound $A(1-\kappa)^{-1 / 2}<1$, and hence essential self-adjointness of $h_{1 / 2}^{\text {rel }}+V$ on $\mathbb{C}^{2} \otimes C_{0}^{\infty}\left(\mathbb{R}^{3}\right)$ follows by Theorem 5.15, Since

$$
\int_{0}^{\infty} d s \int_{\mathbb{R}^{3}} d y(2 \pi s)^{-3 / 2} e^{-\frac{|x-y|^{2}}{2 s}}\left|\log \left(U_{1}(y)\right)\right|=\int_{\mathbb{R}^{3}} \frac{\left|\log \left(\frac{1}{2} \sqrt{b_{1}(y)^{2}+b_{2}(y)^{2}}\right)\right|}{2 \pi|x-y|} d y<\infty,
$$

(5.35) or (5.40) is satisfied. Then (6.4) follows from Theorem 5.11.

qed

We further have the energy comparison inequality following by (6.5). Let

$$
\begin{aligned}
& \tilde{h}_{1 / 2}^{\text {rel }}=\sqrt{2\left(h_{1 / 2}-\mathscr{E}_{h_{1 / 2}^{0}}\right)+m^{2}}-m, \\
& \tilde{h}_{\mathbb{Z}_{2}}^{\text {rel }}=\sqrt{2\left(h_{\mathbb{Z}_{2}}-\mathscr{E}_{h_{\mathbb{Z}_{2}}^{0}}\right)+m^{2}}-m .
\end{aligned}
$$

Note that $h_{1 / 2}-\mathscr{E}_{h_{1 / 2}^{0}} \geq 0$ and $h_{\mathbb{Z}_{2}}-\mathscr{E}_{h_{\mathbb{Z}_{2}}^{0}} \geq 0$ by (5.45) in Corollary 5.13.

Corollary 6.2 (Diamagnetic inequality) Under the assumptions of Theorem 6.1

$$
\left|\left(f, e^{-t\left(\tilde{h}_{\mathbb{Z}_{2}}^{\mathrm{rel}}+V\right)} g\right)\right| \leq\left(|f|, e^{-t\left(h_{\mathbb{Z}_{2}}^{\mathrm{rel}}(0)+V\right)}|g|\right) .
$$

In particular, it follows that

$$
\mathscr{E}_{h_{\mathbb{Z}_{2}}^{\mathrm{rel}}(0)+V} \leq \mathscr{E}_{\tilde{h}_{\mathbb{Z}_{2}}^{\mathrm{rel}}+V}
$$

or equivalently

$$
\mathscr{E}_{h_{1 / 2}^{\mathrm{rel}}(0)+V} \leq \mathscr{E}_{\tilde{h}_{1 / 2}^{\mathrm{rel}}+V}
$$

Generator of $\xi_{T_{t}^{\Psi}}$. In [HL08 and in Lemma 5.10 above we used the $\mathbb{R}^{3} \times \mathbb{Z}_{2^{-}}$ valued joint Brownian and jump process $\xi_{t}=\left(B_{t}, \theta_{N_{t}}\right)$ starting from $\xi_{0}=\left(x, \theta_{\alpha}\right)$ to get the functional integral representation for Schrödinger operators with spin $1 / 2$. The generator of this process is $(1 / 2) \mathrm{p}^{2}+\sigma_{\mathrm{F}}$, where $\sigma_{\mathrm{F}}$ is the fermionic harmonic oscillator defined in terms of the Pauli matrices by

$$
\sigma_{\mathrm{F}}=(1 / 2)\left(\sigma_{3}+i \sigma_{2}\right)\left(\sigma_{3}-i \sigma_{2}\right)-(1 / 2) I=-\sigma_{1} .
$$


Note that $\mathscr{E}_{\frac{1}{2}} \mathrm{p}^{2}+\sigma_{F}=-1$. Similarly, we can identify the generator for the subordinated joint Brownian and jump process

$$
\xi_{T_{t}^{\Psi}}=\left(B_{T_{t}^{\Psi}}, \theta_{N_{T_{t}^{\Psi}}}\right), \quad \Omega_{P} \times \Omega_{N} \times \Omega_{\nu} \rightarrow \mathbb{R}^{3} \times \mathbb{Z}_{2}
$$

starting at $\xi_{0}=\left(x, \theta_{\alpha}\right)$ to be

$$
G=\Psi\left(\frac{1}{2} \mathrm{p}^{2}+\sigma_{\mathrm{F}}+1\right)
$$

This is obtained from the relationship

$$
\sum_{\alpha=1,2} \int \mathbb{E}_{P \times \mu \times \nu}^{x, \alpha, 0}\left[e^{-T_{t}^{\Psi}} \overline{f\left(\xi_{0}\right)} g\left(\xi_{T_{t}^{\Psi}}\right)\right] d x=\left(f, e^{-t G} g\right)
$$

under the identification $L^{2}\left(\mathbb{R}^{3} ; \mathbb{C}^{2}\right) \cong L^{2}\left(\mathbb{R}^{3} \times \mathbb{Z}_{2}\right)$.

Support of magnetic field. Consider the case when $b_{1}(x)-i \theta b_{2}(x)$ vanishes for some $x \in \mathbb{R}^{d}$. In this case it is not clear whether $\int_{0}^{t+}\left|\log \frac{1}{2}\left(b_{1}\left(B_{s}\right)-i \theta_{N_{s-}} b_{2}\left(B_{s}\right)\right)\right| d N_{s}$ is almost surely finite and assumption (4) in Theorem 6.1 holds at all. An example when this is not the case is obtained by choosing $b \in\left(C_{0}^{\infty}\left(\mathbb{R}^{3}\right)\right)^{3}$. To improve Theorem 6.1 we use the ideas of [HL08], where we considered this problem for the Schrödinger operator $\frac{1}{2}(\sigma \cdot(\mathrm{p}-a))^{2}+V$. Let $\delta_{\epsilon}(z)=\left\{\begin{array}{ll}1, & |z|<\epsilon / 2, \\ 0, & |z| \geq \epsilon / 2,\end{array}\right.$ for $z \in \mathbb{C}$ and set $\chi_{\epsilon}(z)=z+\epsilon \delta_{\epsilon}(z)$. We see that

$$
\left|\chi_{\epsilon}\left(-\frac{1}{2}\left(b_{1}(x)-i \theta b_{2}(x)\right)\right)\right|>\epsilon / 2, \quad(x, \theta) \in \mathbb{R}^{3} \times \mathbb{Z}_{2} .
$$

Define $h_{\mathbb{Z}_{2}}^{\epsilon}$ by $h_{\mathbb{Z}_{2}}$ with the off-diagonal part replaced by $\chi_{\epsilon}\left(-\frac{1}{2}\left(b_{1}(x)-i \theta b_{2}(x)\right)\right)$, i.e.,

$$
h_{\mathbb{Z}_{2}}^{\epsilon} f(x, \theta)=\left(h-\frac{1}{2} \theta b_{3}(x)\right) f(x, \theta)+\chi_{\epsilon}\left(-\frac{1}{2}\left(b_{1}(x)-i \theta b_{2}(x)\right)\right) f(x,-\theta) .
$$

We also see that $h_{\mathbb{Z}_{2}}^{\epsilon}$ is self-adjoint on $D(h)$. Define $h_{\mathbb{Z}_{2}}^{\text {rel, } \epsilon}=\sqrt{2 \overline{h_{\mathbb{Z}_{2}}^{\epsilon}}+m^{2}}-m$, where $\overline{h_{\mathbb{Z}_{2}}^{\epsilon}}=h_{\mathbb{Z}_{2}}^{\epsilon}-\mathscr{E}_{h_{\mathbb{Z}_{2}}^{\epsilon}}$ as usual. Since $h_{\mathbb{Z}_{2}}^{\epsilon}$ converges to $h_{\mathbb{Z}_{2}}$ as $\epsilon \downarrow 0$ in uniform resolvent sense, $\mathscr{E}_{h_{\mathbb{Z}_{2}}^{\epsilon}} \rightarrow \mathscr{E}_{h_{\mathbb{Z}_{2}}}$ as $\epsilon \downarrow 0$. Under the assumptions of Theorem 6.1 but without assuming (4) there we are able to show that $h_{\mathbb{Z}_{2}}^{\text {rel, } \epsilon}$ is essentially self-adjoint on $\ell^{2}\left(\mathbb{Z}_{2}\right) \otimes C_{0}^{\infty}\left(\mathbb{R}^{3}\right)$ and the functional integral representation of $h_{\mathbb{Z}_{2}}^{\mathrm{rel}, \epsilon}+V$ holds by (6.4) with

$$
\begin{aligned}
\mathrm{S}_{\mathrm{spin}}^{\Psi}(\epsilon)=\int_{0}^{T_{t}^{\Psi}}\left(\frac{1}{2} b_{3}\left(B_{s}\right) \theta_{N_{s}}-\mathscr{E}_{h_{\mathbb{Z}_{2}}}\right) d s & \\
& \quad+\int_{0}^{T_{t}^{\Psi}+} \log \left(-\chi_{\epsilon}\left(-\frac{1}{2}\left(b_{1}\left(B_{s}\right)-i \theta_{N_{s-}} b_{2}\left(B_{s}\right)\right)\right)\right) d N_{s}
\end{aligned}
$$


instead of $S_{\text {spin }}^{\Psi}$. Moreover, $h_{\mathbb{Z}_{2}}^{\text {rel }, \epsilon}+V$ converges to $h_{\mathbb{Z}_{2}}^{\text {rel }}+V$ on the common core $\ell^{2}\left(\mathbb{Z}_{2}\right) \otimes$ $C_{0}^{\infty}\left(\mathbb{R}^{3}\right)$ so that

$$
\lim _{\epsilon \downarrow 0} \exp \left(-t\left(h_{\mathbb{Z}_{2}}^{\mathrm{rel}, \epsilon}+V\right)\right)=\exp \left(-t\left(h_{\mathbb{Z}_{2}}^{\mathrm{rel}}+V\right)\right)
$$

in strong sense. Hence we have the theorem below.

Theorem 6.3 Take Assumption (A4) and assumptions (1)-(3) in Theorem 6.1. Then the functional integral representation for $h_{\mathbb{Z}_{2}}^{\text {rel }}+V$ is given by

$$
\left(f, e^{-t\left(h_{1 / 2}^{\mathrm{rel}}+V\right)} g\right)=\lim _{\epsilon \downarrow 0} \sum_{\alpha=1,2} \int_{\mathbb{R}^{3}} d x \mathbb{E}_{P \times \mu \times \nu}^{x, \alpha, 0}\left[e^{T_{t}^{\Psi}} \overline{f\left(B_{0}, \theta_{N_{0}}\right)} g\left(B_{T_{t}^{\Psi}}, \theta_{N_{T_{t}^{\Psi}}}\right) e^{\mathrm{S}^{\Psi}(\epsilon)}\right],
$$

where $\mathrm{S}^{\Psi}(\epsilon)=\mathrm{S}_{V}^{\Psi}+\mathrm{S}_{a}^{\Psi}+\mathrm{S}_{\mathrm{spin}}^{\Psi}(\epsilon)$.

\subsection{Spinless case}

Finally consider the spinless case and write

$$
\begin{aligned}
& h^{\mathrm{rel}}=\sqrt{(\mathrm{p}-a)^{2}+m^{2}}-m, \\
& h^{\mathrm{rel}}(0)=\sqrt{\mathrm{p}^{2}+m^{2}}-m .
\end{aligned}
$$

Theorem 6.4 Let Assumption (A3) hold and $V$ be relatively bounded with respect to $\sqrt{\mathrm{p}^{2}+m^{2}}$ with relative bound strictly less than 1 . Then $h^{\mathrm{rel}}+V$ is essentially selfadjoint on $C_{0}^{\infty}\left(\mathbb{R}^{3}\right)$ and

$$
\left(f, e^{-t\left(h^{\mathrm{rel}}+V\right)} g\right)=\int_{\mathbb{R}^{3}} d x \mathbb{E}_{P \times \nu}^{x, 0}\left[\overline{f\left(B_{0}\right)} g\left(B_{T_{t}^{\Psi}}\right) e^{S_{V}^{\Psi}+\mathrm{S}_{A}^{\Psi}}\right] .
$$

Proof. The essential self-adjointness follows from (2) of Corollary 3.13, and (6.9) from Theorem 3.15 .

qed

By Theorem 6.4 we also have the following energy comparison inequality.

Corollary 6.5 (Diamagnetic inequality) Under the assumptions of Theorem 6.4

$$
\left|\left(f, e^{-t\left(h^{\mathrm{rel}}+V\right)} g\right)\right| \leq\left(|f|, e^{-t\left(h^{\mathrm{rel}}(0)+V\right)}|g|\right)
$$

and

$$
\mathscr{E}_{h^{\mathrm{rel}}(0)+V} \leq \mathscr{E}_{h^{\mathrm{rel}}+V}
$$


In the case of $\Psi(u)=\sqrt{2 u+m^{2}}-m$, Assumptions 4.1 and 4.2 are readily satisfied. Furthermore, by Theorem 4.13 we have the result below.

Corollary 6.6 (Hypercontractivity) Let the assumptions of Theorem 6.4 and one of the three equivalent conditions in Proposition 4.5 with $\Psi(u)=\sqrt{2 u+m^{2}}-m$ hold. Then $e^{-t\left(h^{\mathrm{rel}}+V\right)}$ is a bounded operator from $L^{p}\left(\mathbb{R}^{d}\right)$ to $L^{q}\left(\mathbb{R}^{d}\right)$ for all $1 \leq p \leq q \leq \infty$.

\section{A Appendix}

For a given Lévy process $\left(L_{t}\right)_{t \geq 0}$ on a probability space $(\Omega, \mathscr{F}, P)$ the notation $d L_{t}=$ $L_{t}-L_{0}$ is used for its differential. Let $F \in C^{2}(\mathbb{R})$. The differential of the transformed process $d F\left(L_{t}\right)$ can be computed by the following Itô formula.

Proposition A.1 (Itô formula) Let $\mathscr{F}_{t}$ be the natural filtration $\sigma\left(\left(B_{s}, N_{s}^{\beta}\right), 0 \leq s \leq\right.$ $t, \beta=1, \ldots, p)$. Consider

$$
L_{t}^{i}=\int_{0}^{t} f^{i}(s, \omega) d s+\int_{0}^{t} g^{i}(s, \omega) \cdot d B_{s}+\sum_{\beta=1}^{p-1} \int_{0}^{t+} h_{\beta}^{i}(s, \omega) d N_{s}^{\beta}, \quad i=1, \ldots, n
$$

where $f^{i}(\cdot, \omega) \in L_{\text {loc }}^{1}(\mathbb{R})$ a.s, $g^{i} \in \mathscr{E}_{\mathrm{loc}}$ and $h_{\beta}^{i}(s, \omega)$ is adapted with respect to $\mathscr{F}_{t}$, left continuous in $s$ and $\int_{0}^{t+}\left|h_{\beta}^{i}(s, \omega)\right| d N_{s}^{\beta}<\infty$ a.s. Take $F \in C^{2}\left(\mathbb{R}^{n}\right)$. Then for the random process $F\left(L_{t}\right)$ the expression

$$
\begin{aligned}
d F\left(L_{t}\right)= & \sum_{i=1}^{n} \int_{0}^{t} F_{i}\left(L_{s}\right) f^{i}(s) d s+\sum_{i, j=1}^{n} \int_{0}^{t} \frac{1}{2} F_{i j}\left(L_{s}\right) g^{i}(s) \cdot g^{j}(s) d s \\
& +\sum_{i=1}^{n} \int_{0}^{t} F_{i}\left(L_{s}\right) g^{i}(s) \cdot d B_{s}+\sum_{\beta=1}^{p-1} \int_{0}^{t+}\left(F\left(L_{s-}+h_{\beta}(s)\right)-F\left(L_{s-}\right)\right) d N_{s}^{\beta}
\end{aligned}
$$

holds. Here $F_{i}=\partial_{i} F$ and $F_{i j}=\partial_{i} \partial_{j} F$.

Furthermore, the following form of the product rule holds.

Proposition A.2 (Product rule) Let $\left(L_{t}\right)_{t \geq 0}$ and $\left(M_{t}\right)_{t \geq 0}$ be two random processes. Then $d\left(L_{t} M_{t}\right)=d L_{t} \cdot M_{t}+L_{t} \cdot d M_{t}+d L_{t} \cdot d M_{t}$, computed by the rules $d t d t=0$, $d B_{t}^{\mu} d t=0, d B_{t}^{\mu} d B_{t}^{\nu}=\delta_{\mu \nu} d t, d N_{t}^{\alpha} d N_{t}^{\beta}=0, d N_{t}^{\alpha} d t=0$, and $d N_{t}^{\alpha} d B_{t}=0$.

For proofs see, for instance, [W81, LHB09]. 
Acknowledgments: FH acknowledges support of Grant-in-Aid for Scientific Research (B) 20340032 from JSPS and is thankful to Loughborough University, Paris XI University, and IHES, Bures-sur-Yvette, for hospitality. TI acknowledges support of JSPS Grant-in-Aid for Scientific Research (C) 20540161. JL thanks Royal Society for an international travel grant, and the hospitality of Erwin Schrödinger Institute, Vienna, IHES, Bures-sur-Yvette, and Kyushu University, where various parts of this work have been done.

\section{References}

[ALS83] De Angelis, G.F., Jona-Lasinio, G. and Sirugue, M.: Probabilistic solution of Pauli-type equations, J. Phys. A16 (1983), 2433-2444.

[ARS91] De Angelis, G.F., Rinaldi, A. and Serva, M.: Imaginary-time path integral for a relativistic spin-(1/2) particle in a magnetic field, Europhys. Lett. 14 (1991), 95-100.

[AHS78] Avron, J., Herbst, I. and Simon, B.: Schrödinger operators with magnetic fields. I. General interactions, Duke Math. J. 45 (1978), 847-883.

[Bak87] Bakry, D.: Étude des transformations de Riesz dans les variétés riemanniennes à courbure négative minorée, in: Séminaire de probabilités XXI, LNM 1274, Springer, 1987.

[BKM06] Bañuelos, R., Kulczycki, T. and Méndez-Hérnandez, P.J.: On the shape of the ground state eigenfunction for stable processes, Potential Anal. 24 (2006), 205-221.

[BBACT02] Bardou, F., Bouchaud, J.Ph., Aspect, A. and Cohen-Tannoudji, C: Lévy Statistics and Laser Cooling: How Rare Events Bring Atoms to Rest, Cambridge University Press, 2002.

[BF73] Berg, C. and Forst, G.: Potential Theory on Locally Compact Abelian Groups, Springer, 1975.

[Ber99] Bertoin, J.: Subordinators: examples and applications, in: École d'Été de Probabilités de St. Flour XXVII, P. Bernard (ed.), LNM 1717, Springer, 1999, pp. 4-79.

[Boc55] Bochner, S.: Harmonic Analysis and Theory of Probability, University of California Press, 1955.

[BB99] Bogdan, K. and Byczkowski, T.: Potential theory of Schrödinger operator based on fractional Laplacian, Probab. Math. Stat. 20 (2000), 293-335.

[BJ07] Bogdan, K. and Jakubowski, T.: Estimates of heat kernel of fractional Laplacian perturbed by gradient operators, Commun. Math. Phys. 271 (2007), 178-198.

[BG90] Bouchaud, J.Ph. and Georges, A.: Anomalous diffusion in disordered media: statistical mechanisms, models and physical applications, Phys. Rep. 195 (1990), 127-293.

[BHL00] Broderix, K., Hundertmark, D. and Leschke, H.: Continuity properties of Schrödinger semigroups with magnetic fields, Rev. Math. Phys. 12 (2000), 181-225.

[CMS90] Carmona, R., Masters, W.C. and Simon, B.: Relativistic Schrödinger operators: asymptotic behavior of the eigenvalues, J. Funct. Anal. 91 (1990), 117-142.

[CS97] Chen, Z.Q. and Song, R.: Intrinsic ultracontractivity and conditional gauge for symmetric stable processes, J. Funct. Anal. 150 (1997), 204-239. 
[DC00] Demuth, M. and van Casteren, J. A.: Stochastic Spectral Theory for Self-Adjoint Feller Operators. A Functional Integral Approach, Probability and its applications, Birkhäuser, 2000.

[EK95] Eberlein, E. and Keller, U.: Hyperbolic distributions in finance, Bernoulli 1 (1995), 281-299.

[GV81] Gaveau, B. and Vauthier, J., Intégrales oscillantes stochastiques: l'équation de Pauli, J. Funct. Anal. 44 (1981), 388-400.

[GR07] Grzywny, T. and Ryznar, M.: Two-sided optimal bounds for Green functions of half-spaces for relativistic $\alpha$-stable process, Potential Anal. 28 (2008), 201-239.

[HS78] Herbst, I.W. and Sloan, D.: Perturbation of translation invariant positivity preserving semigroups on $L^{2}\left(\mathbb{R}^{n}\right)$, Trans. AMS 236 (1978), 325-360.

[Hir09] Hiroshima, F.: Functional integral representation of relativistic Pauli-Fierz model, in preparation.

[HL08] Hiroshima, F. and Lörinczi, J.: Functional integral representation of the Pauli-Fierz model with spin 1/2, J. Funct. Anal. 254 (2008), 2127-2185.

[HS09] Hiroshima, F. and Sasaki, I.: Ground state of relativistic Nelson model, in preparation.

[HS95] Hislop, P.D. and Sigal, I.M., Introduction to Spectral Theory, AMS 113, Springer, 1995.

[Huf69] Huff, B.: The strict subordination of differential processes, Sankhyā Ser. A 31 (1969), 403412.

[Ich87] Ichinose, T.: The nonrelativistic limit problem for a relativistic spinless particle in an electromagnetic field, J. Funct. Anal. 73 (1987), 233-257.

[Ich94] Ichinose, T.: Some results on the relativistic Hamiltonian: self-adjointness and imaginary-time path integral, in: Differential Equations and Mathematical Physics, Proc. of the Int. Conference, University of Alabama at Birmingham, March 13-17, 1994, International Press, Boston, MA, 1995, pp. 102-116.

[ITa86] Ichinose, T. and Tamura, H.: Imaginary-time path integral for a relativistic spinless particle in an electromagnetic field, Commun. Math. Phys. 105 (1986), 239-257.

[IW81] Ikeda, N. and Watanabe, S.: Stochastic Differential Equations and Diffusion Processes, NorthHolland Mathematical Library 24, North-Holland, 1981.

[Kat76] Kato, T.: Perturbation Theory for Linear Operators, 2nd edition, Springer, 1976.

[Kan77] Kanter, M.: Unimodality and dominance for symmetric random vectors, Trans. AMS 229 (1977), 65-85.

[KS06] Kulczycki, T. and Siudeja, B.: Intrinsic ultracontractivity of the Feynman-Kac semigroup for relativistic stable processes, Trans. AMS 358 (2006), 525-5057.

[LS81] Leinfelder, H. and Simader, C.G.: Schrödinger operators with singular magnetic potentials, Math. Z. 176 (1981), 1-19.

[Lie73] Lieb, E.H.: Bounds on the eigenvalues of the Laplacian and Schrödinger operators, Bull. AMS 82 (1976), 751-753.

[Lie80] Lieb, E.H.: The number of bound states of one-body Schrödinger operators and the Weyl problem, Proc. of the Math. Soc. Symposia in Pure Math. 36 (1980), 241-252.

[Lor09a] Lörinczi, J.: Exponential integrability of some rough functionals, in: Proc. Sixth Seminar on Stochastic Analysis, Ascona, 2008, Birkhäuser, to appear, 2009. 
[Lor09b] Lörinczi, J.: Exponential functionals of twice iterated integrals with respect to subordinated Brownian motion, in preparation, 2009.

[LHB09] Lörinczi, J., Hiroshima, F. and Betz, V.: Feynman-Kac-Type Theorems and Gibbs Measures on Path Space. With Applications to Rigorous Quantum Field Theory, Walter de Gruyter, 2009 (to appear).

[MK04] Metzler, R. and Klafter, J.: The restaurant at the end of the random walk: recent developments in the description of anomalous transport by fractional dynamics, J. Phys. A 37 (2004), $161-208$.

[RS78] Reed, M. and Simon, B.: Methods of Modern Mathematical Physics, vol II: Fourier Analysis, Self-Adjointness, Academic Press, 1975.

[Ryz02] Ryznar, M.: Estimates of the Green function for relativistic $\alpha$-stable process, Potential Anal. 17 (2002), 1-23.

[Sat99] Sato, K.I.: Lévy Processes and Infinite Divisibility, Cambridge University Press, 1999.

[Shi87] Shigekawa, I.: Eigenvalue problems for the Schrödinger operator with the magnetic field on a compact Riemannian manifold, J. Funct. Anal. 75 (1997), 92-127.

[Sim78] Simon, B.: A canonical decomposition for quadratic forms with applications to monotone convergence theorems, J. Funct. Anal. 28 (1978), 377-385.

[Sim79] Simon, B.: Maximal and minimal Schrödinger forms, J. Operator Th. 1 (1979), 37-47.

[Sim82] Simon, B.: Schrödinger semigroups, Bull. AMS 7 (1982), 447-526; Erratum: Bull. AMS 11 (1984), 426 .

[Sim04] Simon, B.: Functional Integration and Quantum Physics, 2nd ed., AMS Chelsea Publishing, 2004.

[SV09] Song, R. and Vondraček, Z.: Potential theory of subordinate Brownian motion, in: Potential Analysis of Stable Processes and its Extensions, LNM 1980, Springer, 2009.

[Yam78] Yamazato, M.: Unimodularity of infinitely divisible distributions of class L, Ann. Prob. 6 (1978), 523-531.

[Wol78] Wolfe, S. J.: On the unimodality of multivariate symmetric distribution functions of class $L$, J. Mult. Anal. 8 (1978), 141-145. 